Chapman University

Chapman University Digital Commons

Food Science (MS) Theses

Dissertations and Theses

Summer 7-2019

\title{
L-Cysteine Effects on Chlorogenic Acid Quinone-Amino Acid Induced Greening and Browning: Mechanism and Effects on Antioxidant Capacity
}

Yundi Liang

Chapman University, yuliang@chapman.edu

Follow this and additional works at: https://digitalcommons.chapman.edu/food_science_theses

Part of the Food Chemistry Commons

\section{Recommended Citation}

Liang, Y. (2019). L-cysteine effects on chlorogenic acid quinone-amino acid induced greening and browning: Mechanism and effects on antioxidant capacity. Master's thesis, Chapman University. https://doi.org/10.36837/chapman.000092

This Thesis is brought to you for free and open access by the Dissertations and Theses at Chapman University Digital Commons. It has been accepted for inclusion in Food Science (MS) Theses by an authorized administrator of Chapman University Digital Commons. For more information, please contact laughtin@chapman.edu. 
L-cysteine effects on chlorogenic acid quinone-amino acid induced greening and browning: mechanism and effects on antioxidant capacity

\author{
A Thesis by \\ Yundi Liang \\ Chapman University \\ Orange, California \\ Food Science Program \\ Schmid College of Science and Technology \\ Submitted in partial fulfillment of the requirements for the degree of \\ Master of Science in Food Science
}

July 2019

Committee in charge:

Lilian Were, Ph.D., Committee Chair

Rosalee Hellberg, Ph.D.

Cedric Owens, Ph.D. 
The thesis of Yundi Liang is approved.

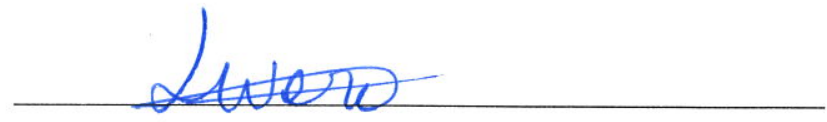

Lilian Were, Ph.D. Committee Chair
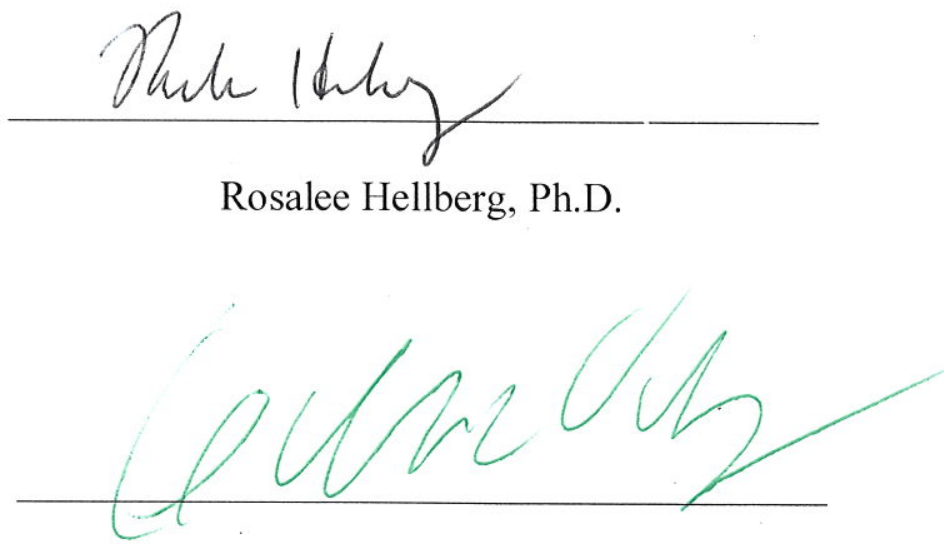

Cedric Owens, Ph.D.

July 2019 
L-cysteine effects on chlorogenic acid quinone-amino acid induced greening and browning: mechanism and effects on antioxidant capacity

Copyright $@ 2019$

by Yundi Liang 


\section{ACKNOWLEDGEMENTS}

The author wishes to acknowledge Michelle Tu, Olive Dahm, and Kristen Shiba for helping me with baking cookies, Xiangren Chen for helping me with absorbance data analysis and SAS software coding, Robyne Kelly for teaching me being nice to myself, Dr. Maduka Obga and Dr. Allegra Liberman-Martin for help on organic chemistry, my great lab peers for being a great team, and the most important-------my dearest thesis advisor Dr. Lilian Were for always having faith in me and continuous encouragements even when I had a thought of quitting. 


\section{DEDICATION}

This thesis is dedicated to my loving and amazing parents for the continuous supports, and encouragements. 


\begin{abstract}
L-cysteine effects on chlorogenic acid quinone-amino acid induced greening and browning: mechanism and effects on antioxidant capacity

by Yundi Liang
\end{abstract}

The formation of green trihydroxy benzacridine (TBA) derivatives when chlorogenic acid (CGA) quinones and amino acids react can be visually unappealing in some applications where CGA containing ingredients are used. Cysteine was studied as an amino acid anti-greening strategy, because cysteine-CGA conjugates are colorless. Buffered CGA: lysine: cysteine solutions at pH 8.0 and 9.0 were prepared and incubated for a maximum of $48 \mathrm{~h}$ at ambient temperature. Color intensity was periodically monitored using a UV-Vis spectrophotometer. Quantification and identification of conjugate formation were conducted by HPLC and LC-MS, while Antioxidant capacity was assessed by Trolox Equivalent Antioxidant Capacity and Folin-Ciocalteu reagent reducing capacity assays. More intense greening was detected at higher $\mathrm{pH}$. Lysyl amineCGA conjugates were identified as the predominant precursor of green TBA. Concentration-dependent cysteine inhibition of CGA-lysine greening was primarily by redox diphenol regeneration when $\mathrm{pH}$ was below cysteinyl thiol pKa 8.3 while primarily by forming cysteinyl-CGA conjugates when $\mathrm{pH}$ was above 8.3. Visible greening was fully inhibited with a cysteine: lysine 1:1 molar ratio in pH 9 CGA: Lys: Cys solutions, indicating that cysteinyl thiol was a stronger nucleophile than $\varepsilon$-lysyl amine to react with CGA o-quinones. Mono- and di-cysteine-CGA conjugates contributed to antioxidant capacity. Cysteine concentration, $\mathrm{pH}$ and incubation time all significantly affected color intensities and antioxidant capacity $(\mathrm{p}<0.0001)$. In addition, a predominant mechanism of 
green trihydroxy benzacridine derivatives was postulated, with lysyl amine-CGA conjugate identified as the predominant precursor ion. The results complement knowledge of polyphenol-protein interactions, which can be applicable to develop chlorogenic acid-containing sunflower seed products in the future.

Keywords: Antioxidant capacity, browning, chlorogenic acid quinones, cysteine, greening, Maillard reaction 


\section{TABLE OF CONTENTS}

1. Introduction

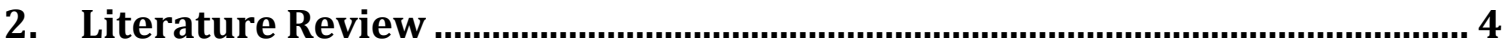

2.1. Chlorogenic acid quinone-amino acid induced greening ...................................... 4

2.2. Cysteine as a dough conditioner in baked products ........................................ 9

2.2.1. Anti-greening strategies in chlorogenic acid containing baked products........... 9

2.3. Cysteine effects on heated reducing sugar-sunflower seed protein mixture (a

Maillard reaction model) ............................................................................................... 11

2.3.1. Cysteine inhibition of Maillard browning ..................................................... 11

2.3.2. Cysteine enhancement of meaty flavor and antioxidant compounds

formation through Maillard reaction ......................................................................... 12

2.4. Cysteine-chlorogenic acid quinone reaction and effects on antioxidant capacity.

2.5. Cysteine inhibition of caffeic acid oxidation ................................................ 13

2.6. Competing pathway of $\varepsilon$-lysyl amine and cysteinyl thiol groups for

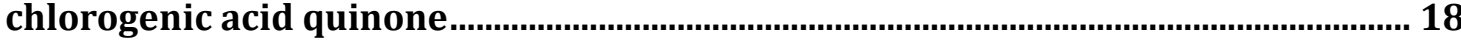

2.6.1. Potential effects of cysteine inhibition reaction on color................................ 18

2.6.2. Potential effects of cysteine inhibition reaction on antioxidant capacity . 20

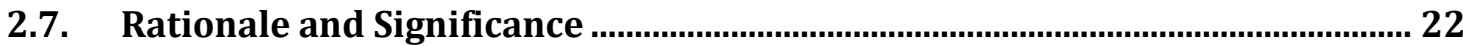

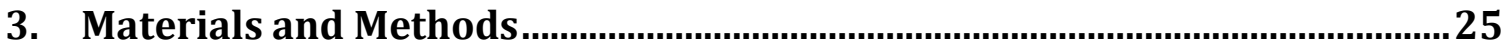

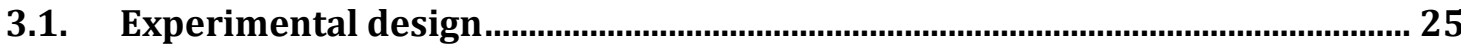

3.2. Solution and buffer preparation and UV-Vis scans of model solutions .......... 26

3.3. Folin-Ciocalteu Reagent reducing and Trolox equivalent antioxidant capacity (TEAC) assays of CGA: lysine: cysteine solutions............................................................ 28

3.4. Unreacted CGA and CGA conjugates quantification by HPLC .............................. 28

3.5. Identification of CGA-cys adducts and TBA derivatives in solution model by LC-MS 29

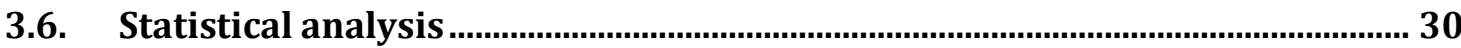

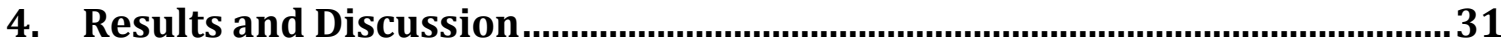

4.1. Color intensities in unbuffered CGA: Lys: Cys solutions ........................................ 31

4.2. Effects of cysteine concentration on color intensities......................................... 33

4.3. Effect of $\mathrm{pH}$ on brown and green color intensities ........................................... 40

4.4. Formation of reaction products over incubation............................................42

4.5. Cysteine effects on Trolox equivalents antioxidant capacity (TEAC) and Folin-Ciocalteu Reagent reducing Capacity (FCRC) in CGA: Lys: Cys solutions ......... 52

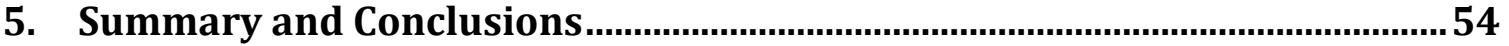

6. Recommendations for future studies ............................................................... 55

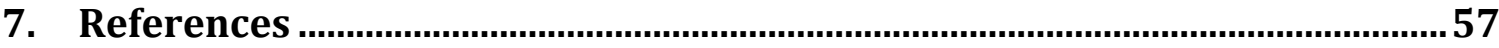

8. APPENDIX: Preliminary studies with cookies.................................................62 


\section{LIST OF TABLES}

Table 1 Chlorogenic acid $o$-quinones reaction with dietary amino acids and their effects on pigmentation.

Table 2 Selected studies modifying cookie dough ingredients and their effect on chlorogenic acid quinone-amino acid induced greening and subsequent effects on phenolic profile.

Table $3 \mathrm{pH}$ of unbuffered pH 9 CGA: Lys: Cys solutions over $48 \mathrm{~h}$ incubation at ambient

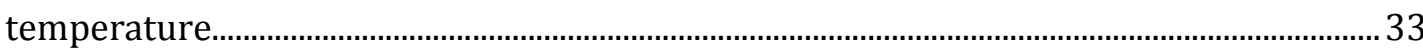

Table 4 General linear model effect and interaction of $\mathrm{pH}$, cysteine concentration and incubation time on color intensities and antioxidant capacity............................................40

Table 5 Relative abundance of eluted compounds at 1.60 and 1.75 minutes by HPLC peak

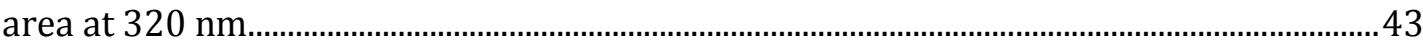

Table 6 Identification of reaction products in $\mathrm{pH} 8$ and 9 buffered chlorogenic acid /CGA:

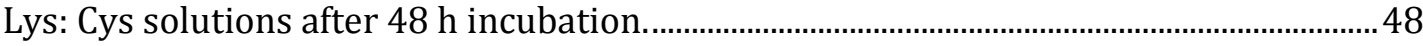

Table 7 Hunter $\mathrm{L}^{*}, \mathrm{a}^{*}$, or $\mathrm{b}^{*}$ values of sunflower butter cookies internal transections as a function of storage time when L-cysteine was increased from 0 to $3.72 \mathrm{mM}$....................66

Table 8 Progression of surface and internal color in sunflower butter cookies as a function of time and L-cysteine concentration during uncovered storage at ambient temperature 


\section{LIST OF FIGURES}

Figure 1 Colorless chlorogenic acid oxidation to yellow CGA ortho-quinones: primary amines added to CGA o-quinones, dimerized with another CGA and cyclized to green trihydroxy benzacridine derivatives. R1 and R2 refer to quinic acid (174 Da) and lysine (131 Da) moieties.

Figure 2 Caffeic acid (left) and chlorogenic acid (right) dissolved in pH 9 phosphate buffer at ambient temperature $\left(\sim 22^{\circ} \mathrm{C}\right)$.

Figure 3 Cysteine effects on caffeic acid oxidation at $\mathrm{pH}$ above pKa of 8.3: brown and yellow routes show caffeic acid oxidation and browning formation with and without cysteine respectively. With added cysteine (brown route on the right), caffeic acid takes a longer time (slower rate) and more oxygen to accumulate the same dark brown intensity as via yellow route does, adapted from Cilliers and Singleton (1990)......................................15

Figure 4 Comparison of initial chlorogenic acid/ caffeic acid ester oxidation products.........19

Figure 5 Postulated mechanism and effects on antioxidant capacity of $\varepsilon$-lysyl amine and cysteinyl thiol groups competing for chlorogenic acid o-quinone, which induced greening and browning respectively in aqueous solution at alkaline $\mathrm{pH}$ (cysteinyl thiol pKa), adapted from Yabuta et al. (2001), Pierpoint (1966) and Penalver et al. (2002). 22

Figure 6 Experimental design for cysteine effects on color intensities, reaction products and antioxidant capacity as a function of $\mathrm{pH}$ and cysteine concentration in chlorogenic acid: L-lysine: L-cysteine solutions.

Figure 7 Conjugation analysis on 1: 1: 0.22 .55 mM CGA: 5.09 mM L-Lysine: 0-5.09 mM Lcysteine solutions

Figure 8 Browning measured by absorbance at $410 \mathrm{~nm}(\mathrm{~A}, \mathrm{C})$ and greening measured by absorbance at $680 \mathrm{~nm}(\mathrm{~B}, \mathrm{D})$ in unbuffered $\mathrm{pH} 8$ and 9 CGA: lysine: cysteine solutions as a function of reaction time for $48 \mathrm{~h}$ when cysteine increased from 0 to $1.486 \mathrm{mM}$...32

Figure 9 Browning measured by absorbance at $410 \mathrm{~nm}(\mathrm{~A})$ and greening measured by absorbance at $680 \mathrm{~nm}$ (B) in pH 7.75, 8 and 9 buffered CGA: lysine: cysteine solutions as a function of reaction time for $48 \mathrm{~h}$ when cysteine increased from 0 to $1.486 \mathrm{mM}$. The data presented were means of 3 replicates. Means with the same letter after $48 \mathrm{~h}$ were not significantly different.

Figure $10 \mathrm{pH} 8$ (A to F) and pH 9 (G to L) buffered CGA: Lys: Cys solutions with $0 \mathrm{mM}$ (control), $1.7 \mathrm{mM}$ and $5.1 \mathrm{mM}$ cysteine incubated for $24 \mathrm{~h}$ at ambient temperature.....34

Figure 11 Browning measured by absorbance at $410 \mathrm{~nm}$ (A, B), greening measured by absorbance at $680 \mathrm{~nm}(\mathrm{C}, \mathrm{D})$ and photos representing visual appearance with corresponding correlation coefficients between -0.6408 and -0.9919 of $\mathrm{pH} 8$ and 9 buffered chlorogenic acid (CGA): lysine: cysteine solutions incubated at ambient temperature when cysteine increased from 0 to $5.091 \mathrm{mM}$. The data were means \pm standard deviations of 3 replicates. Means with the same letter after $48 \mathrm{~h}$ were not significantly different. 
Figure 12 Postulated cysteine inhibition of chlorogenic acid-lysine greening at alkaline $\mathrm{pH}$. $\mathrm{R}_{1}, \mathrm{R}_{2}$, and $\mathrm{R}_{3}$ refers to a quinic acid, lysine and cysteine moiety, respectively. The CGA quinone derivatives may be monomer, dimer or polymers. Dash-line framed route was adapted from Yabuta et al. (2001), which was a potential but less predominant route in our study.

Figure 13 Postulated mechanism of cysteine regenerating chlorogenic acid hydroquinone dimer by redox reaction.

Figure 14 HPLC chromatograms at $320 \mathrm{~nm}(\mathrm{~A}, \mathrm{~B}), 450 \mathrm{~nm}(\mathrm{C}, \mathrm{D})$, and $680 \mathrm{~nm}(\mathrm{E}, \mathrm{F})$ of pH 8 and 9 buffered CGA: Lys: Cys solutions after $48 \mathrm{~h}$ incubation at ambient temperature.44

Figure 15 Mass spectrometric chromatograms and chemical structures of identified compounds in pH 8 and 9 chlorogenic acid: Lys: Cys solutions.

Figure 16 Trolox equivalents antioxidant capacity (A) and gallic acid equivalent reducing capacity (B) as a function of cysteine concentration (0 - 5.091 mM) in buffered CGA: Lys: Cys solutions.

Figure 17 Greening measured by absorbance at $680 \mathrm{~nm}$ (A, B), browning by absorbance at 410 $\mathrm{nm}(\mathrm{C}, \mathrm{D})$ of $\mathrm{pH} 8$ and 9 buffered chlorogenic acid (CGA): lysine: glutathione solutions incubated at ambient temperature for $24 \mathrm{~h}$ when glutathione increased from 0 to $5.091 \mathrm{mM}$.

Figure 18 Internal greening (\%) of sunflower butter cookies as a function of storage time during uncorvered storage at ambient temperature when L-cysteine was increased from 0 to $3.72 \mathrm{mM}$. 


\begin{abstract}
ABBREVIATIONS
CGA - Chlorogenic acid (5-Caffeoylquinic acid, PubChem CID:1794427)

CGA-Q - Chlorogenic acid quinone (Chlorogenoquinone, PubChem CID:73759913)

Cys - L-Cysteine (PubChem CID: 5862)

Cys-HCI - L-Cysteine hydrochloride (PubChem CID: 60960)

HPLC - High performance liquid chromatography

FCRC - Folin-Ciocalteu reagent Reducing Capacity

LCMS - Liquid chromatography mass spectrophotometry

Lys - L-Lysine (PubChem CID: 5962)

MRPs - Maillard Reaction Products

TBA - Trihydroxy benzacridine

TEAC - Trolox Equivalents Antioxidant Capacity
\end{abstract}




\section{Logic Model}

Situation:

I Intense greening occurs when primary amines nucleophilically attack chlorogenic acid (CGA) quinones to form green trihydroxy benzacridine (TBA) derivatives.

7 L-cysteine reacts with CGA o-quinone monomers to produce colorless cysteine-CGA adducts indicating its potential anti-greening effect in CGA quinone-amino acid reactions.

7 L-cysteine is an affirmed generally recognized as safe FDA-approved dough strengthener with a usage limit of 90 ppm in yeast-leavened baked goods and baking mixes (21 CFR 184.1271) and as “Quantum satis” by the European Food Safety Authority.

Need: Elucidate if and how cysteine competitively inhibits CGA quinone-lysine induced greening.

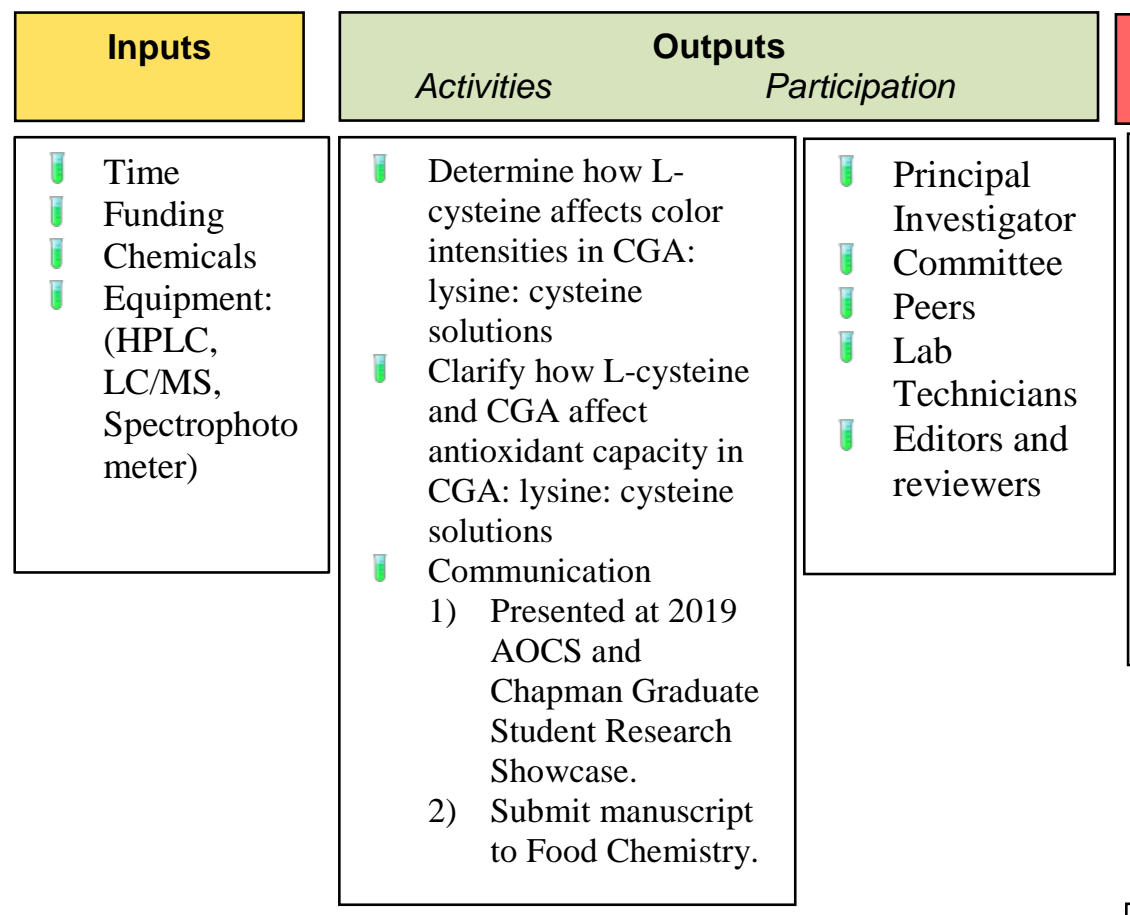

\section{Assumptions:}

As $\alpha$-amine of cysteine is less nucleophilic than thiol group,

cysteinyl $\alpha$-amine was assumed not to react with CGA-quinones at all tested pHs.

\begin{tabular}{|c|c|c|c|c|c|}
\hline \multicolumn{6}{|c|}{ Outcomes } \\
\hline 『 & $\begin{array}{l}\text { Cysteine effects of } \\
\text { A) pH ( } 7.75,8 \text { and } \\
\text { 9) } \\
\text { B) L-cysteine } \\
\text { concentrations } \\
\text { on CGA: lysine: } \\
\text { cysteine solutions on } \\
\text { greening, browning } \\
\text { and antioxidant } \\
\text { capacity. }\end{array}$ & J & $\begin{array}{l}\text { Correlations between } \\
\text { incubation time and } \\
\text { L-cysteine } \\
\text { concentration with } \\
\text { color intensities in } \\
\text { CGA: lysine: cysteine } \\
\text { solutions at pH 7.75, } 8 \\
\text { and 9. } \\
\text { Identification of } \\
\text { CGA-amino acid } \\
\text { (lysine or cysteine) } \\
\text { conjugates in CGA: } \\
\text { lysine: cysteine } \\
\text { solutions. } \\
\text { Effects of L-cysteine } \\
\text { concentration on } \\
\text { conjugate formation } \\
\text { antioxidant capacity. }\end{array}$ & VI & $\begin{array}{l}\text { Mechanism on how L- } \\
\text { cysteine reacts with CGA in } \\
\text { the presence of lysine } \\
\text { forming TBA } \\
\text { chromophores. } \\
\text { Determination of difference } \\
\text { between antioxidant } \\
\text { capacity of free CGA and } \\
\text { thiol-CGA conjugates. } \\
\text { Improved understanding of } \\
\text { effects of pH, incubation } \\
\text { and concentration of } \\
\text { substrates of thiol addition } \\
\text { between thiols (from free } \\
\text { amino acids or protein } \\
\text { residues) and } \\
\text { hydroxycinnamic acid } \\
\text { quinones, such as } \\
\text { chlorogenic acid quinones. }\end{array}$ \\
\hline
\end{tabular}

\section{External Factors:}

7 Cysteinyl-CGA conjugates might be brown as increased browning was observed at highest cysteine concentration.

I Brown CGA quinones might conceal the color of cysteinyl-CGA conjugates, which were noted as colorless conjugates in literature. 


\section{Introduction}

Hydroxycinnamic acids such as CGA with C3-C6 backbones are non-flavonoid phenolic compounds ubiquitous in plants (Rong, 2010), possessing potential health benefit in controlling oxidative and inflammatory stress (Tosovic, Markovic, Markovic, Mojovic, \& Milenkovic, 2017). Chlorogenic acids (CGA) are hydroxycinnamic acid esters of caffeic and quinic acids, which constitute up to $75 \%$ of total phenolics in sunflower oilseeds and 67\% in green coffee beans (Budryn \& Rachwal-Rosiak, 2013). However, antioxidant capacity is lowered in CGA-bearing food products because CGA can be thermally degraded or participate in oxidative colorization reactions. Besides oxidized CGA induced browning, green pigmentation is also observed in alkalized sunflower seed meal because oxidized CGA interacts with amino acids to form green trihydroxy benzacridine (TBA) derivatives. Chlorogenic acid can be oxidized to brown CGA quinones by polyphenol oxidase (PPO) or non-enzymatically at alkaline pH by oxygen. Electrophilic CGA quinones bond with nucleophilic primary $\alpha$-amine groups through nucleophilic substitution and Diels-Alder-Dehydro reaction to form green pigmented trihydroxy benzacridine (TBA) derivatives (Namiki, Yabuta, Koizumi, \& Yano, 2001). This intense greening also occurs in sunflower butter cookies. For consumers who are allergic to peanuts, sunflower seed butter is a good alternative as it provides similar quantities of macronutrients but is not one of the eight major allergens. Unlike peanut butter cookies, cookies made from sunflower butter contain oxidized CGA o-quinones which react with protein, to produce greening. Green sunflower butter cookies which might be unappealing to consumers had lowered antioxidant capacity (Liang \& Were, 2018b). 
Oxidation of CGA in sunflower butter cookies is attributed to alkaline $\mathrm{pH}$ by baking soda. Incorporating acidic ingredients into cookie dough formulation can inhibit CGA quinone induced greening by lowering pH (Atonfack, Ataman, \& Were, 2019; Liang, Were, \& Hanh Lan, 2018; Liang \& Were, 2018b; Rogers, Lan, Vu, \& Were, 2018a). This is because acidic $\mathrm{pH}$ prevents deprotonated phenolic ions from oxidizing to form CGA quinones (Cilliers \& Singleton, 1989). However, up to 51\% intersectional greening occurred within $24 \mathrm{~h}$ post baking when sunflower butter cookies were formulated with acidic dairy ingredients (Liang et al., 2018). In addition to potential sour taste due to acidic dairy ingredients, no anti-greening method based on ingredient substitution or changes in temperature was reported to fully inhibit visible greening.

Since CGA o-quinones and primary amine groups are primary substrates for Maillard browning and TBA greening (Yabuta, Koizumi, Namiki, Hida, \& Namiki, 2001), de-phenolization and acidic pH were studied to obtain non-green sunflower protein (SFP). Sunflower protein extracted from de-phenolized seed meal at pH 9 had more than 50\% protein extractability than acidic protein extraction at pH 6 (GonzalesPerez, Merck, Vereijken, Koningsveld, \& Gruppen, 2002). However, de-phenolization requires use of organic solvents, such as methanol and 2-proponal, which make up a huge part of the waste in industry and thus may lead to negative public impression on SFP, as sustainability is one major factors driving growth in the plant-based protein market (Eardley, 2018; Hartman, 2019).

Lysyl-e-amine was identified as the most reactive substrate to form green TBA derivatives (Bongartz et al., 2016) due to its high nucleophilicity. L-cysteine, a US FDAapproved GRAS dough strengthener, reacts with CGA o-quinones to form a colorless 
cysteine-CGA conjugate (Bongartz et al., 2016; Schilling, Sigolotto, Carle, \& Schieber, 2008). Lund and Ray (2017) and Pierpoint (1966) reported that cysteinyl thiol had a higher affinity for CGA $o$-quinones than $\alpha$-amine groups, so cysteinyl thiol is hypothesized to conjugate with $o$-quinones before $\alpha$-lysyl amine. However, lysine has a more nucleophilic $\varepsilon$-amine (pKa 10.5) whose affinities for CGA quinones was not reported in any studies compared to cysteinyl thiol (pKa 8.3) at alkaline pH. Given the high reactivity of cysteinyl thiol group and $\varepsilon$-amine group of lysine, it is hard to predict cysteine's anti-greening effect on CGA quinone-amino acid induced greening, which was attributed to intensely green pigmented CGA quinone-lysine adducts (Liang \& Were, 2018b).

The long-term research goal is to provide an alternative anti-greening strategy for alkaline protein extraction from sunflower meal, which does not require removal of phenolics. The overall goal was accomplished by determining how the individual and combined influences of cysteine concentration, $\mathrm{pH}$ and incubation time affected CGA quinone-amino acid induced greening in CGA-lysine solutions, followed with elucidation of cysteine’s anti-greening mechanism.

This study is innovative as it provides an alternative anti-decolorization strategy for greening, which also enhances antioxidant capacity. Compared to other anti-greening strategies, such as acidic protein extraction and de-phenolization (Gonzales-Perez et al., 2002), adding cysteine during alkaline protein extraction might retard green pigmentation while formation of cysteinyl-CGA conjugates might also inhibit protein oxidation. In addition, this study complements knowledge of polyphenol-protein interactions, which can be applied chlorogenic acid-containing sunflower protein products in the future, as 
sunflower protein was recognized as one of the top 6 plant-based protein ingredients (Ferrer, 2019).

\section{Literature Review}

\subsection{Chlorogenic acid quinone-amino acid induced greening}

Hydroxycinnamic acids with C3-C6 backbones are non-flavonoid phenolic compounds found in high concentrations in fruits, vegetables, grains and seeds. While caffeic acid and its esters in fruits and vegetables such as chlorogenic acid, are mainly found in free forms (Rong, 2010; Schlichtherle-Cerny \& Amado, 2002), in grains and seeds, they are often bound to cellulose, lignin and proteins which can only be released by acid, alkaline or enzymatic hydrolysis (Budryn \& Rachwal-Rosiak, 2013; GonzalesPerez et al., 2002).

Chlorogenic acids are hydroxycinnamic acid esters of caffeic and quinic acids (Rong, 2010). In plants, 5- caffeoylquinic acid (5-CQA) is the most common (up to 75\% of total phenolics in sunflower oilseeds and 67\% in green coffee beans) of the three chlorogenic acid isomers (3, 4, or 5-CQA), (Budryn \& Rachwal-Rosiak, 2013). Due to its electrophilic nature, CGA is a naturally-occurring antioxidant, possessing potential health benefit in controlling oxidative and inflammatory stress by hydrogen atom transfer, radical adduct formation, and sequential proton loss electron transfer (Ningjian \& David, 2015; Tosovic et al., 2017). Hydroxycinnamic acids when thermally degraded or when they participate in polyphenol oxidase induced enzymatic browning have lowered antioxidant capacity because free phenolics were oxidized (Altunkaya \& Gökmen, 2009).

Thermal processing produces aromas by formation of a 1,5- $\gamma$-quino-lactone from 
CGA during coffee roasting, or green pigmentation in alkalinized sunflower seed meal when oxidized CGA interacts with amino acids (Bongartz et al., 2016; Ningjian \& David, 2015).

Alkaline $\mathrm{pH}$, amine groups and CGA-quinones composed of $o$-diphenyl and caffeate moieties are essential for green pigmentation by TBA derivatives (Yabuta et al., 2001). Chlorogenic acid can also be oxidized to CGA quinones by polyphenol oxidase (PPO). Electrophilic CGA quinones bond with nucleophilic primary $\alpha$-amine groups through covalent addition to form pigmented trihydroxy benzacridine (TBA) derivatives (Figure 1). Due to their high electrophilicity, CGA quinones polymerize with each other to form brown compounds when free $\alpha$-amine groups are absent (Namiki et al., 2001).
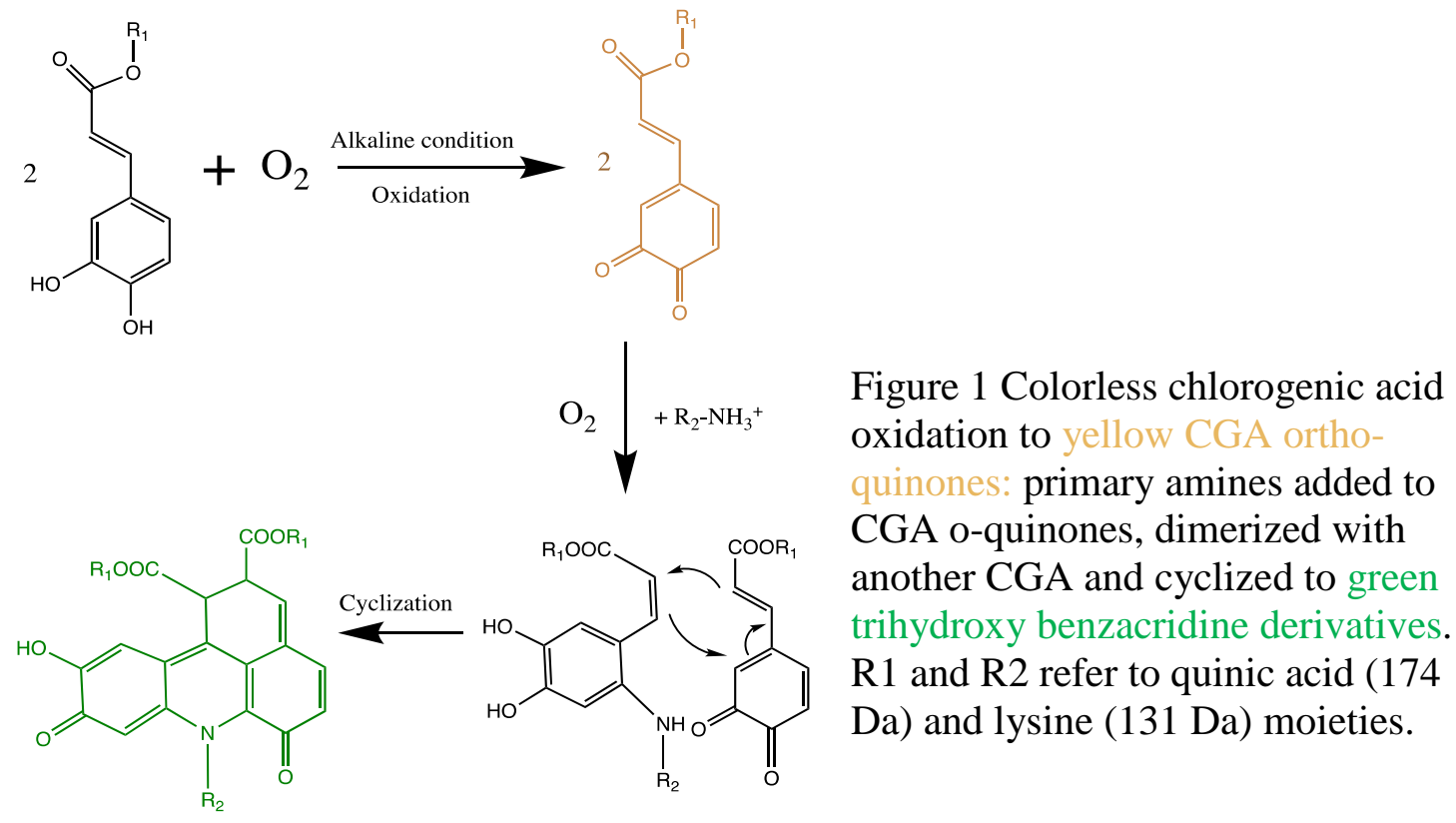

Ortho-quinones non-enzymatically react with amino acids or amine groups of proteins via three pathways: (1) Strecker degradation to form volatile compounds such as furans, thiophenes and pyrazines, which contributes to nutty, meaty and caramel-like flavors to the system; (2) imine formation, which enhances formation of melanoidins; or 
(3) nucleophilic addition to form pigmented S/N-quinonyl amino acids adducts/TBA derivatives (Bittner, 2006; Namiki et al., 2001).

To generate green TBA derivatives, dimerized CGA first oxidizes to highly reactive ortho-quinone, and then the CGA o-quinone covalently bonds to an available primary amine group from amino acids or alkyl amine, followed by nucleophilic cyclization and loss of a water molecule (Bongartz et al., 2016; Namiki et al., 2001; Yabuta et al., 2001). Formation of TBA derivatives is promoted by alkaline conditions because nucleophilic addition is favored at higher pH (Hayase, Kim, \& Kato, 1984).

Quinones and primary amine groups are primary substrates to form TBA derivatives (Rawel \& Rohn, 2010). Primary amines covalently bond with CGA quinones at the electrophilic C6 position by nucleophilic addition, forming N-quinonyl amino acid adducts, cyclized to benzacridine structures and then oxidized to TBA derivatives. High reactivity of $\varepsilon$-amine group of lysine and aromatic side chain of tyrosine was reported by Prigent et al. (2008) and Rawel and Rohn (2010). Protein cross-link might occur once phenolic compounds were depleted in a phenol-amino acid system. Sulfur atom from methionine and imidazole group of tryptophan cross link with CGA given the nucleophilicity from sulfur and nitrogen elements (Rawel \& Rohn, 2010). Rawel and Rohn (2010) also reported that the benzacridine structure cyclization products possessed redox potential, which formed oxidized blue or green pigmented TBA derivatives.

Chlorogenic acid o-quinones and primary amines are essential for green trihydroxy benzacridine derivatives formation. Among the 20 common amino acids (Table 1), 5 amino acids do not result in green pigmentation upon reacting with chlorogenic acid $o$-quinones. 
Table 1 Chlorogenic acid o-quinones reaction with dietary amino acids and their effects on pigmentation.

\begin{tabular}{|c|c|c|c|c|}
\hline Amino acids & $\begin{array}{l}\text { Classification based } \\
\text { on structure }\end{array}$ & $\begin{array}{l}\text { Induce greening } \\
\text { when react with } \\
\text { CGA }\end{array}$ & Pigmentation reaction & References \\
\hline \multicolumn{5}{|c|}{ Non-greening inducing amino acids } \\
\hline Proline & $\begin{array}{l}\text { Secondary amino } \\
\text { acid }\end{array}$ & $\begin{array}{l}\text { No, due to lack } \\
\text { of primary } \\
\text { amine group }\end{array}$ & $\begin{array}{l}\text { No green or brown color } \\
\text { forms. }\end{array}$ & $\begin{array}{l}\text { (Bongartz } \\
\text { et al., 2016; } \\
\text { Jackson \& } \\
\text { Kendal, } \\
\text { 1949; } \\
\text { Pierpoint, } \\
\text { 1969) }\end{array}$ \\
\hline $\begin{array}{l}\text { Ser, Thr, } \\
\text { Cys }\end{array}$ & $\begin{array}{l}\text { Primary amino } \\
\text { acids with } \\
\text { nucleophilic side } \\
\text { chain }\end{array}$ & $\begin{array}{l}\text { No, because } \\
\text { their } \\
\text { nucleophilic side } \\
\text { chains are more } \\
\text { reactive } \\
\text { nucleophiles } \\
\text { than their } \alpha- \\
\text { amine groups. }\end{array}$ & $\begin{array}{l}\text { Serine and threonine induced } \\
\text { reddish-brown, due to } \beta \text { - } \\
\text { hydroxy side group. } \\
\text { Cysteine reacted with CGA } \\
\text { and formed colorless } \\
\text { conjugates. }\end{array}$ & $\begin{array}{l}\text { (Bongartz } \\
\text { et al., 2016; } \\
\text { Pierpoint, } \\
\text { 1966, 1969; } \\
\text { Schilling et } \\
\text { al., 2008; } \\
\text { Yabuta et } \\
\text { al., 2001) }\end{array}$ \\
\hline Trp & $\begin{array}{l}\text { Primary amino } \\
\text { acids without } \\
\text { nucleophilic side } \\
\text { chain }\end{array}$ & $\begin{array}{l}\text { No, due to } \\
\text { presence of an } \\
\text { indole ring. }\end{array}$ & $\begin{array}{l}\text { Red CGA quinone- } \\
\text { tryptophan conjugates were } \\
\text { formed due to presence of an } \\
\text { indole ring. }\end{array}$ & $\begin{array}{l}\text { (Bongartz } \\
\text { et al., 2016; } \\
\text { Schilling et } \\
\text { al., 2008) }\end{array}$ \\
\hline \multicolumn{5}{|c|}{ Greening inducing amino acids } \\
\hline $\begin{array}{l}\text { Asn, Gln, } \\
\text { Asp, Glu, } \\
\text { His, Lys, } \\
\text { Arg, Phe, } \\
\text { Tyr, } \\
\text { Gly,Ala, } \\
\text { Val, Leu, } \\
\text { Ile, Met }\end{array}$ & $\begin{array}{l}\text { Primary amino } \\
\text { acids without } \\
\text { nucleophilic side } \\
\text { chain }\end{array}$ & Yes & $\begin{array}{l}\text { Chlorogenic acid } o \text {-quinones } \\
\text { nucleophilic conjugated with } \\
\alpha \text {-amines and cyclized to } \\
\text { form green trihydroxy } \\
\text { benzacridine derivatives. }\end{array}$ & $\begin{array}{l}\text { (Bongartz } \\
\text { et al., 2016; } \\
\text { Namiki et } \\
\text { al., 2001; } \\
\text { Pierpoint, } \\
\text { 1969; } \\
\text { Prigent et } \\
\text { al., 2008; } \\
\text { Rawel \& } \\
\text { Rohn, } \\
\text { 2010; } \\
\text { Schilling et } \\
\text { al., 2008; } \\
\text { Yabuta et } \\
\text { al., 2001) }\end{array}$ \\
\hline
\end{tabular}

Secondary amines such as proline do not react with CGA o-quinones to form green TBA derivatives by amine addition. Jackson and Kendal (1949) observed redpurple pigmentation in catechol-proline solution with tyrosinase at $\mathrm{pH} 7$ to 8 , which 
faded within an hour and ended with brown coloration due to phenolic quinones polymerization, indicating that electrophilic phenolic quinones might form unstable conjugates with secondary amines.

Primary amino acids with nucleophilic side chains such as serine, threonine and cysteine, substituted electrophilic CGA o-quinones with their side groups, as their side groups are more nucleophilic than $\alpha$-amines and thus have a higher affinity to CGA $o$ quinones (Lund \& Ray, 2017; Pierpoint, 1966). Tryptophan, a primary amino acid with a nucleophilic secondary amine as its side group, reacts with CGA $o$-quinones by amine addition to form a red TBA derivative due to the presence of an indole ring (Bongartz et al., 2016).

Intense greening measured at $680 \mathrm{~nm}$ has been observed with TBA derivatives formed with lysine (Bongartz et al., 2016; Yabuta et al., 2001), due to higher reactivity of lysine of its $\varepsilon$-amine. TBA derivatives formed between CGA quinones and lysine or glycine were also identified as stable natural green pigments by Iacomino et al. (2017). Iacomino et al. (2017) also confirmed with UHPLC-MS that both $\alpha$ - and $\varepsilon$ - amine group of lysine could react with CGA quinones to form green TBA derivatives, consistent with results from Schilling et al. (2008) with N-terminally blocked lysine reacting with CGA dimers and results from Prigent et al., (2008) that reaction products of CGA quinones reacting with lysine had a lower relative electrospray ionization signal intensity than with $\mathrm{N}$-terminally blocked lysine, indirectly confirming the $\varepsilon$-amine group of lysine as a preferred substrate for CGA quinones to form CGA quinone-lysine adducts due to higher nucleophilicity of lysyl $\varepsilon$-amine group. 


\subsection{Cysteine as a dough conditioner in baked products}

L-cysteine/L-cysteine $\mathrm{HCl}$ is affirmed as a generally recognized as safe (GRAS) ingredient by U.S. Food and Drug Administration (21 CFR. 184. 1271/ 1272). European Food Safety Authority permits the use of L-cysteine (E920) at levels indicated by quantum satis under regulation No 1129/2011. “Quantum satis” indicates that no maximum level is specified.

\subsubsection{Anti-greening strategies in chlorogenic acid containing baked products}

Intense greening in CGA containing food products occurs when nucleophilic amine group(s) of amino acids covalently bond with CGA quinones to form green trihydroxy benzacridine (TBA) derivatives. Changes in sunflower butter cookie dough formulation, baking temperature, and storage conditions can lower greening (Table 2). All aforementioned de-greening methods were not able to completely inhibit and thus cysteine was investigated as an alternative de-greening strategy. Bongartz et al. (2016) found that cysteine-CGA solutions were colorless at $\mathrm{pH}$ 9, consistent with findings by Richard-Forget et al. (1992) that CGA-cys conjugates were colorless ortho-diphenolic compounds. 
Table 2 Selected studies modifying cookie dough ingredients and their effect on chlorogenic acid quinone-amino acid induced greening and subsequent effects on phenolic profile.

\begin{tabular}{|c|c|c|c|c|}
\hline \multirow[t]{2}{*}{ Ingredient(s) } & \multicolumn{2}{|c|}{ Effects on } & \multirow[t]{2}{*}{ Limitations } & \multirow[t]{2}{*}{ Citations } \\
\hline & greening & phenolic profile & & \\
\hline $\begin{array}{l}\text { Acidulants: } \\
\text { Citric acid and GDL* } \\
\text { differing in } \mathrm{pH}\end{array}$ & $\begin{array}{l}\text { Acidic } \mathrm{pH} \\
\text { lowered } \\
\text { greening in } \\
\text { refrigerated } \\
\text { cookie dough }\end{array}$ & $\begin{array}{l}\text { Acidic dough retained } \\
\text { higher phenolic content } \\
\text { during storage. }\end{array}$ & $\begin{array}{l}\text { Inapplicable to } \\
\text { industrial } \\
\text { alkaline } \\
\text { processing }\end{array}$ & $\begin{array}{l}\text { (Atonfack } \\
\text { et al., } \\
\text { 2019) }\end{array}$ \\
\hline \multirow[t]{2}{*}{$\begin{array}{l}\text { Liquid sweeteners } \\
\text { differing in } \\
\text { i) } \mathrm{pH} \text { and } \\
\text { ii)Moisture content } \\
\text { and water activity }\end{array}$} & $\begin{array}{l}\text { More alkaline } \\
\text { pH maple } \\
\text { sweeteners } \\
\text { enhanced } \\
\text { greening }\end{array}$ & $\begin{array}{l}\text { More alkaline sweeteners } \\
\text { (maple syrup) lowered } \\
\text { free phenolic content in } \\
\text { cookies than acidic } \\
\text { sweeteners (honey and } \\
\text { agave syrup). }\end{array}$ & $\begin{array}{l}\text { Solid } \\
\text { sweeteners are } \\
\text { preferred for } \\
\text { industrial } \\
\text { processing due } \\
\text { to lower cost. } \\
\text { Use of honey } \\
\text { could increase } \\
\text { Maillard } \\
\text { glycation } \\
\text { products. }\end{array}$ & $\begin{array}{l}\text { (Liang \& } \\
\text { Were, } \\
\text { 2018a, } \\
\text { 2018b) }\end{array}$ \\
\hline & $\begin{array}{l}\text { High moisture } \\
\text { content and } \\
\text { water activity/a } \mathrm{a}_{\mathrm{w}} \\
\text { enhanced } \\
\text { greening }\end{array}$ & $\begin{array}{l}\text { High moisture content } \\
\text { lowered free phenolic } \\
\text { content in cookies. }\end{array}$ & $\begin{array}{l}\text { Greening } \\
\text { accumulated } \\
\text { within } 4 \mathrm{~h} \\
\text { storage. }\end{array}$ & $\begin{array}{l}\text { (Liang \& } \\
\text { Were, } \\
\text { 2018b) }\end{array}$ \\
\hline $\begin{array}{l}\text { Acidic dairy ingredients } \\
\text { differing in } \mathrm{pH} \text { and } \mathrm{a}_{\mathrm{w}}\end{array}$ & $\begin{array}{l}\text { Acidic dairy } \\
\text { ingredients } \\
\text { lowered } \\
\text { greening. }\end{array}$ & $\begin{array}{l}\text { Acidic dairy ingredients } \\
\text { maintained higher } \\
\text { antioxidant capacity. }\end{array}$ & $\begin{array}{l}\text { Inapplicable to } \\
\text { alkaline } \\
\text { industrial } \\
\text { processing. } \\
\text { Sourness from } \\
\text { acidic } \\
\text { ingredients } \\
\text { may affect } \\
\text { flavor. }\end{array}$ & $\begin{array}{l}\text { (Liang et } \\
\text { al., 2018) }\end{array}$ \\
\hline $\begin{array}{l}\text { Plant-based egg replacers } \\
\text { differing in } \\
\text { moisture content }\end{array}$ & $\begin{array}{l}\text { Higher moisture } \\
\text { enhanced } \\
\text { greening. }\end{array}$ & $\begin{array}{l}\text { High moisture content } \\
\text { increased the loss of free } \\
\text { phenolic content and } \\
\text { protein oxidation in } \\
\text { cookies. }\end{array}$ & $\begin{array}{l}\text { Moisture } \\
\text { content of } \\
\text { cookies is } \\
\text { typically } \\
\text { around 3\% } \\
\text { (w.b.). }\end{array}$ & $\begin{array}{l}\text { (Rogers } \\
\text { et al., } \\
\text { 2018a) }\end{array}$ \\
\hline $\begin{array}{l}\text { L-Cysteine } \\
\text { concentrations }\end{array}$ & $\begin{array}{l}\text { Lowered } \\
\text { greening in } \\
\text { solutions when } \\
\text { equal molarities } \\
\text { of cysteine and } \\
\text { lysine are } \\
\text { present but did } \\
\text { not degreen } \\
\text { cookies }\end{array}$ & $\begin{array}{l}\text { Increased antioxidant } \\
\text { capacity. }\end{array}$ & $\begin{array}{l}\text { Cysteine is a } \\
\text { GRAS } \\
\text { ingredient } \\
\text { with negative } \\
\text { public image } \\
\text { in US and } \\
\text { Europe }\end{array}$ & $\begin{array}{l}\text { Current } \\
\text { study; } \\
\text { (Radiana } \\
\text { Maria, } \\
\text { Ciprian } \\
\text { Nicolae, } \\
\text { \& Stela, } \\
\text { 2013) }\end{array}$ \\
\hline
\end{tabular}

*GDL = glucono delta lactone 


\subsection{Cysteine effects on heated reducing sugar-sunflower seed protein mixture (a Maillard reaction model)}

\subsubsection{Cysteine inhibition of Maillard browning}

Cysteine was identified by Ashoor and Zent (1984) as a low browning inducing amino acid when compared with high browning inducing amino acids such as lysine and glycine, and intermediate browning inducing amino acids such as proline, leucine, isoleucine. Among the 20 amino acids, L-cysteine had the lowest browning /absorbance at $420 \mathrm{~nm}$ when heated with D-ribose, D-glucose, D-fructose or $\alpha$-lactose at $121^{\circ} \mathrm{C}$ for 10 mins (Ashoor \& Zent, 1984).

The inhibitive effect of L-cysteine on Schiff base formation $\left(\mathrm{A}_{294 \mathrm{~nm}}\right)$ and Maillard browning ( $\mathrm{A}_{420 \mathrm{~nm}}$ ) was reported by Zhang et al. (2018) in xylose and soybean peptide mixtures with added L-cysteine. Although $\mathrm{pH}$ of the reaction solutions were pre-adjusted to 7.6, the final $\mathrm{pH}$ significantly decreased as the temperature increased from $80^{\circ} \mathrm{C}$ to $120^{\circ} \mathrm{C}$, and $\mathrm{pH}$ decreased more after heating for 2 hours in reaction mixtures with added L-cysteine (Zhang et al., 2018). The decreased pH might be due to thermal degradation of xylose, amino acids and peptides to formic and acetic acids as heating temperature increased from $80^{\circ} \mathrm{C}$ to $120^{\circ} \mathrm{C}$. Zhang et al. (2018) suggested that cysteine might hinder carbonyl-amine cross-linking to form Schiff bases in addition to accelerated $\mathrm{pH}$ decrease due to acids formation, which suppressed melanoidins formation. An inhibitive effect of cysteine on Maillard browning in protein mixtures was also reported by Zeng et al. (2017) and Eric et al. (2013). When Zeng et al. (2017) compared two reducing agents: Lcysteine and L-ascorbic acid in a Maillard reaction system consisting of enzymatically derived chicken hydrolysate with an 1:1 xylose: ribose reducing sugar mixture, lower 
absorbance at $294 \mathrm{~nm}$ and $420 \mathrm{~nm}$ for Schiff base and browning were reported when Lcysteine was increased from $0 \%$ to $0.5 \%(\mathrm{~m} / \mathrm{v})$, while Schiff bases formation and browning intensity were not concentration dependent as L-cysteine was increased from $0.5 \%$ to $2.0 \%(\mathrm{~m} / \mathrm{v})$. Eric et al. $(2014 ; 2013)$ found that adding cysteine to a heated sunflower protein isolate and xylose mixture significantly lowered melanoidins due to competitive consumption of carbonyl from CGA, in addition to inhibition of peptidedicarbonyl cross-links.

\subsubsection{Cysteine enhancement of meaty flavor and antioxidant compounds formation through Maillard reaction}

During thermal processing, protein degradation had limited effect on $\mathrm{pH}$ decrease in a heated protein hydrolysate mixture without reducing sugar, so $\mathrm{pH}$ decrease was mainly attributed to formation of organic acids by Maillard reaction (Lan et al., 2010). Eric et al., (2014) reported that final pH, CGA content, formation of melanoidins and high (>5 kDa) and medium (1-5 kDa) molecular weight crosslink products significantly decreased when cysteine was added to a pH 7.4 sunflower peptide and xylose mixture thermally treated at $80^{\circ} \mathrm{C}$ to $140^{\circ} \mathrm{C}$ for $2 \mathrm{~h}$. Peptide degradation to smaller fractions and free amino acids due to a pH reduction, was enhanced when cysteine was added because deprotonation of cysteine further lowered the $\mathrm{pH}$. Since sugars and free amino acids/ smaller peptides crosslinked to form volatile compounds whose formation is highly dependent on composition of amino acids during MR, adding cysteine increased sulfurcontaining furans, thiophenes, and aliphatic sulfur compounds while formation of nitrogen-containing compounds like pyrazines and pyrroles decreased, giving a less caramel but more meaty overall flavor. 


\subsection{Cysteine-chlorogenic acid quinone reaction and effects on antioxidant capacity}

Cysteine's thiol (R-SH) side group makes it a highly reactive nucleophilic amino acid. Unlike the thiol-ether sulfur group of methionine, the cysteinyl thiol can be ionized to a highly nucleophilic thiolate. When thiol groups react with quinones, there are two potential pathways: thiol oxidation by electrophilic quinones to form disulfide bonds, which can diminish quinone-induced browning; or covalent attachment of thiol groups to quinones forming mono- or di-thiol-substituted quinones by thiol-quinone addition reaction, which can prevent CGA quinone-amino acid induced greening ( $\mathrm{Li}$, Wolfgang, \& Jurgen, 2005; Snell \& Weissberger, 1939). Thiols covalently bond to quinones and methyl substituted quinones by nucleophilic addition. Direct oxidation of thiols by excess quinones to disulfide at acidic or slightly alkaline conditions ( $\mathrm{pH} 4$ to 7.5 ) was due to the reducing capacity possessed by cysteinyl thiols. At neutral $\mathrm{pH}$, one or two thiol groups covalently bond to phenolics via Michael addition (Schilling et al., 2008). With CGA quinones oxidized by fungal tyrosinase or alkaline oxidation, $\mathrm{N}$-acetyl-L-cysteine conjugated with CGA monomer through covalent addition at one or two reactive sites, indicated regeneration from CGA-quinone back to CGA.

With added cysteine, sunflower protein-xylose MR system had significantly higher antioxidant capacity (Eric et al., 2013), as assessed by both Folin-Ciocalteu reagent reducing capacity (FCRC) and 2,2-diphenyl-1-picryl-hydrazyl-hydrate (DPPH) free radical assays.

\subsection{Cysteine inhibition of caffeic acid oxidation}

Cilliers and Singleton (1990) found that cysteinyl thiol delayed caffeic acid nonenzymatic oxidation rate and increased total oxygen uptake when different concentrations 
of cysteine (4 -16 mM) were added to caffeic acid aqueous solutions at $\mathrm{pH} 7$ to 14 . Brown pigmentation $\left(\mathrm{A}_{420} \mathrm{~nm}\right)$ at $\mathrm{pH}$ 8, 9, 10 and 11 strongly correlated with fullyoxidized caffeic acid monitored by HPLC at $210 \mathrm{~nm}$ in $2 \mathrm{mM}$ : $8 \mathrm{mM}$ caffeic acid: cysteine solutions. Cysteine's protective effects on caffeic acid were observed at $\mathrm{pH} 8$ to 11 by delaying oxidation of caffeic acid. Caffeic acid dissolves at alkaline $\mathrm{pH}$ forming a bright yellow caffeic acid mono-quinone, which then oxidatively couples with another caffeic acid to form dark brown caffeic acid dimer-quinone (Figure 2).

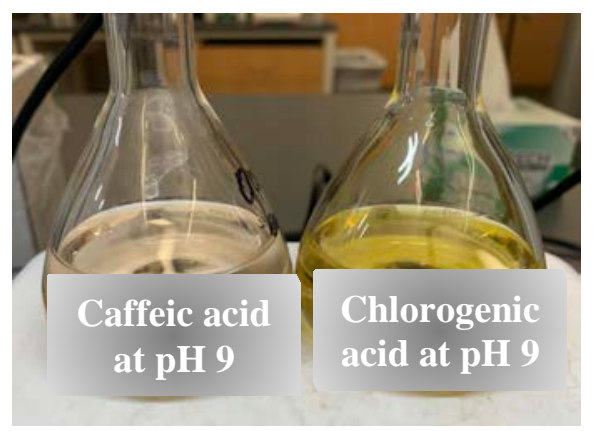

Figure 2 Caffeic acid (left) and chlorogenic acid (right) dissolved in $\mathrm{pH} 9$ phosphate buffer at ambient temperature $\left(\sim 2{ }^{\circ} \mathrm{C}\right)$

Browning was delayed at $\mathrm{pH} 8$ with precipitated cystine, because caffeic acid mono-quinones were reduced back to hydroquinones while cysteine was oxidized to cystine. As pH increased above cysteine’s thiol pKa (8.3), cysteine slowed oxidation by phenol-regenerative thiol conjugation instead of redox reaction. Above $\mathrm{pH}$ 8.3, initial oxidation produced caffeic acid mono-quinones which are highly electrophilic and could be stabilized by polymerization or by reacting with nucleophiles such as thiols and amines (Cilliers \& Singleton, 1989). At pH 9 to 11, thiolates bond with caffeic acid mono-quinones to form thiol-ether mono-hydroquinones which could undergo further oxidation to thiol-ether mono-quinones (Figure 3). Unoxidized caffeic acids then oxidatively couple with thiol-ether mono-quinones to form thiol-ether dimerhydroquinones that eventually oxidize to dark brown thiol-ether dimer-quinones. When thiol-ether mono-hydroquinones are present, unoxidized phenols are more prone to 
oxidative coupling with thiol-ether mono-hydroquinones due to a lower redox potential of thiol-ether mono-hydroquinones compared to unsubstituted caffeic acid monoquinone.

Phenol-thiol mixtures such as caffeic acid and cysteine, have higher total oxygen uptake than phenols alone with oxygen (Figure 3). Cysteine alone does not consume oxygen under the same conditions ( $\mathrm{pH}$ above 8.3 with oxygen), while addition of cysteine to phenols not only increased the total oxygen consumption but also prolonged the oxidation reaction (took a longer time to achieve maximum browning intensity). Browning plateaued at $\mathrm{pH} 9$ to 11, indicating that increasing $\mathrm{pH}$ above 9 did not enhance browning with the same substrate concentrations.

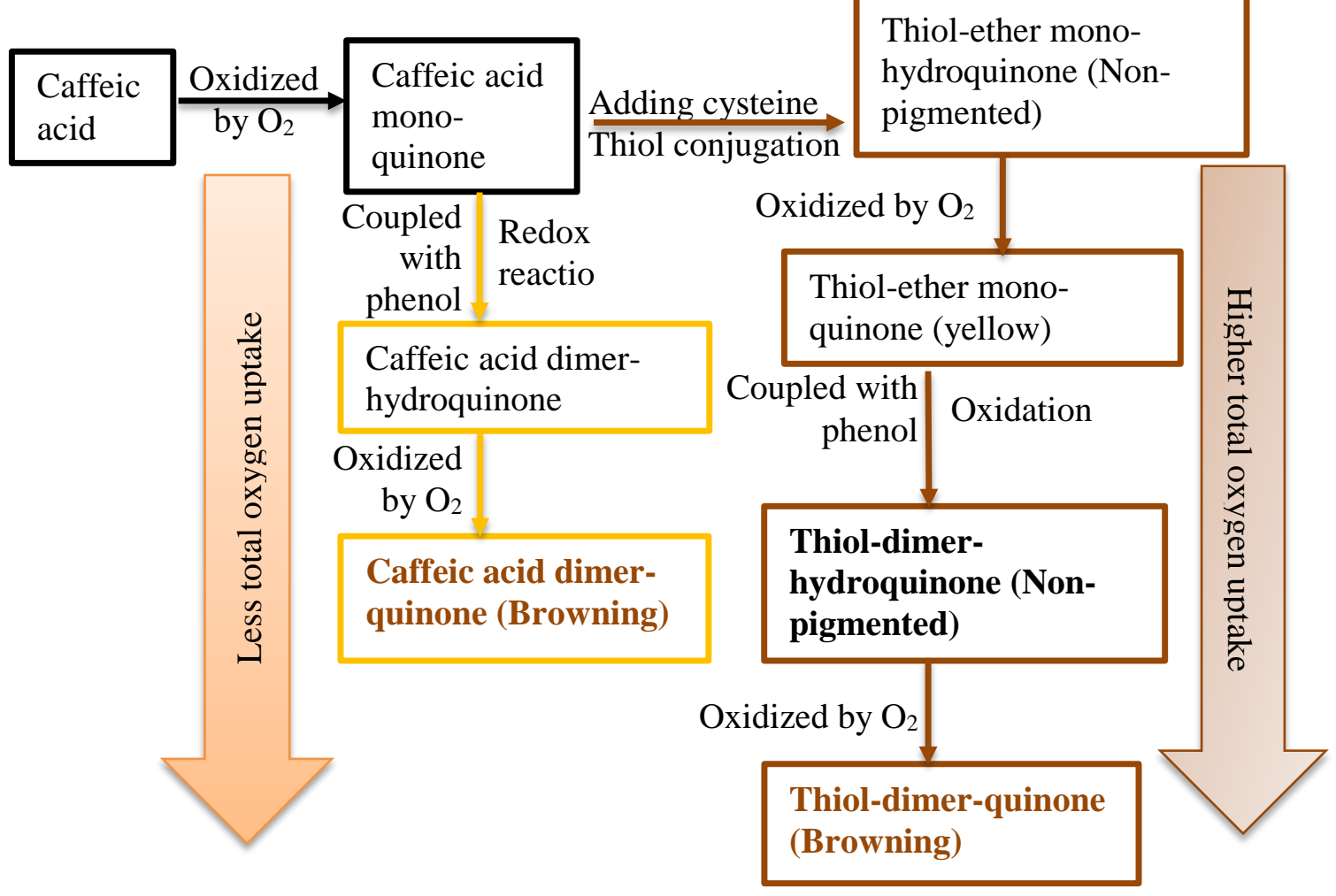

Figure 3 Cysteine effects on caffeic acid oxidation at $\mathrm{pH}$ above $\mathrm{pKa}$ of 8.3: brown and yellow routes show caffeic acid oxidation and browning formation with and without cysteine respectively. With added cysteine (brown route on the right), caffeic acid takes a longer time (slower rate) and more oxygen to accumulate the same dark brown intensity as via yellow route does, adapted from Cilliers and Singleton (1990). 
Caffeic acid oxidation induced browning was faster as $\mathrm{pH}$ increased from 9 to 11 with or without cysteine. Caffeic acid phenolate ion concentration increased as $\mathrm{pH}$ increased above its phenolic pKa of 9.5. Since phenolate ion concentration was the limiting factor in caffeic acid oxidation at alkaline conditions, plateaued dark brown intensities in shorter period of time was expected as $\mathrm{pH}$ increased from 9 to 11 (Cilliers \& Singleton, 1989).

Cysteine concentration was independent of its caffeic acid protective effect against browning, with $2 \mathrm{mM}$ caffeic acid reacting with cysteine (1- $16 \mathrm{mM})$ at $\mathrm{pH} 10$ (Cilliers \& Singleton, 1990), which was consistent with the regenerative protecting mechanism of phenols shown in Figure 3. Final brown intensities accumulated to the same maximum as cysteine concentration increased from 1 to $16 \mathrm{mM}$, while caffeic acid took a longer time to be fully oxidized to form dark brown colors. Increased cysteine concentration delayed caffeic acid oxidation at $\mathrm{pH}$ 10. Yellowness and thiol-ether hydroquinone reaction intermediates monitored by HPLC at $200 \mathrm{~nm}$, 2-S-cysteinylcaffeic acid (2-CCA), 5-S-cysteinylcaffeic acid (5-CCA) and 2,5-di-S-cysteinylcaffeic acid (DDCA) in pH 10 caffeic acid and cysteine solutions reached maximums until cysteine was fully depleted, and subsequent oxidation of caffeic acid was rapid. Since the 2position of caffeic acid phenol ring was most electrophilic (Cilliers \& Singleton, 1990), nucleophilic thiol conjugation occurred preferentially to form 2-CCA; thus, decreased caffeic acid monitored at $200 \mathrm{~nm}$ was strongly correlated with primary reaction intermediate 2-CCA at all cysteine concentrations. DDCA increased as cysteine concentration increased under the same conditions. The observation that DDCA formed maximally after forming 2- and 5- S-cysteinyl-caffeic acid with a formation rate more 
dependent on cysteine concentrations reinforced Cilliers and Singleton’s (1989, 1990) postulation that DDCA formed from either 2- or 5- S-cysteinyl-caffeic acid.

When caffeic acid and cysteine concentrations were constant, the maximum 2CCA concentrations remained the same when $\mathrm{pH}$ was increased from 9 to 13, which formed slowest at $\mathrm{pH} 9$ and fastest at $\mathrm{pH}$ 13. 2-S-cysteinylcaffeic acid forming faster as $\mathrm{pH}$ increased also indicated that thiol-ether hydroquinone reaction intermediates formed by highly nucleophilic thiolate attacking electrophilic caffeic acid mono-quinone, which was limited by phenolate ion concentration. 2-S-Cysteinylcaffeic acid was much lower at $\mathrm{pH} 7$ and 8 due to $\mathrm{pH}$ being below cysteine thiol pKa (8.3), indicating thiolates were one of the required thiol conjugation substrates instead of sulfhydryl.

Cilliers and Singleton (1990) found that cystine started to precipitate after $12 \mathrm{~h}$ incubation in $\mathrm{pH} 7$ caffeic acid-cysteine solutions. Adding more cysteine after $12 \mathrm{~h}$ did not reverse browning, confirming that yellow and dark brown oxidation products were two different irreversible compounds as initial yellow oxidation products could be reduced by cysteine. In addition, reducing agents such as mercaptoethanol, dithiothreitol and their mixtures confirmed that more sulfhydryl groups exhibited greater delay on caffeic oxidation induced browning. Overall, caffeic acid and its derivatives (chlorogenic acid and caftaric acid) oxidized with cysteine in a similar pathway due to the structural similarity of C3-C6 phenol backbone. Cysteine reacted with PPO or alkaline pH induced caffeic acid o-quinones primarily by redox phenol regeneration with cystine precipitation when $\mathrm{pH}$ was below cysteinyl thiol $\mathrm{pKa}$ of 8.3. When $\mathrm{pH}$ was above 8.3, cysteine reacted with o-quinones by thiol-conjugation: thiyl radical nucleophilic added to yellow electrophilic $o$-quinones to form colorless thiol-ether conjugates which were subject to 
secondary oxidation. Caffeic acid o-quinones had a primary conjugation site at 2-position of phenol due to its highest electrophilicity. Eventually, increased yellowness $\left(\mathrm{A}_{420} \mathrm{~nm}\right)$ was observed when excess cysteine was added (Cilliers \& Singleton, 1990; Murata, Sugiura, Sonokawa, Shimamura, \& Homma, 2002; Penalver, Rodriguez-Lopez, GarciaMolina, Garcia-Canovas, \& Tudela, 2002; Pierpoint, 1969; Richard, Goupy, Nicolas, Lacombe, \& Pavia, 1991; Richard-Forget et al., 1992).

\subsection{Competing pathway of $\varepsilon$-lysyl amine and cysteinyl thiol groups for chlorogenic acid quinone}

\subsubsection{Potential effects of cysteine inhibition reaction on color}

In pH 9 phosphate buffer, chlorogenic acid solution is golden brown while caffeic acid is not (Figure 2) because pH is above pKa of CGA (8.42) but not caffeic acid (9.5). Phenolic pKa of caffeic and chlorogenic acids are 9.5 and 8.42, respectively (Maegawa, Sugino, \& Sakurai, 2007) and phenolate ion concentration positively correlated with $o$ quinone formation (Cilliers \& Singleton, 1989). Hence, limited caffeic acid o-quinone was formed in $\mathrm{pH} 9$ phosphate buffer.

Similar to caffeic acid oxidation products discussed in section 2.5, chlorogenic acid/ 5-caffeoquinic acid, has a mono-ortho-quinone (Lund, Heinonen, Baron, \& Estevez, 2011; Murata et al., 2002), contradicting the structure of chlorogenic acid dimer-oquinone as the initial CGA oxidation product which was described as the primary substrate of CGA quinone amino acid induced greening (Bongartz et al., 2016). Lund et al. (2011) confirmed that an imino-quinone formed when nucleophilic $\alpha$-amine nucleophilic added to electrophilic CGA quinone. Namiki and others (2001) also observed an ethyl caffeate radical, a semi-quinone intermediate, by Electro-spin 
Resonance (ESR) in pH 9.5 aerated ethyl-caffeate- $\alpha$-alanine solutions (Figure 4).

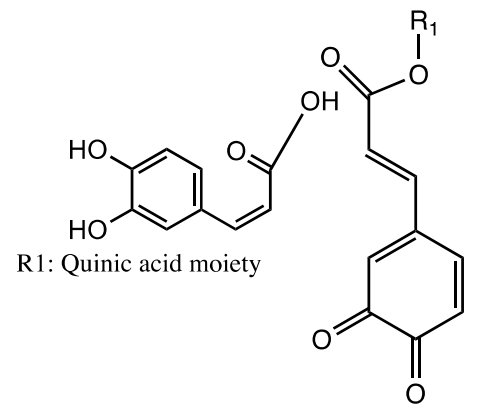

CGA o-quinone (Murata et al., 2002; Lund et al., 2011)

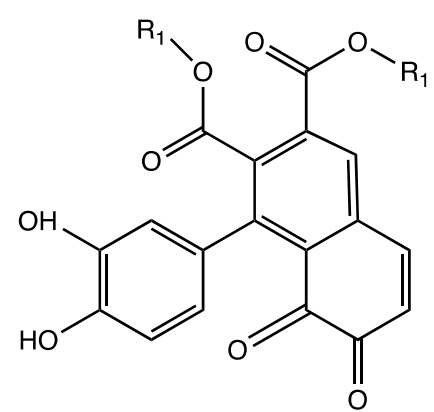

CGA o-quinone (Bongartz et al., 2016)<smiles>CCOC(=O)CC=C1C=CC(=O)C([O-])=C1</smiles>

Ethyl Caffeate radical (Namiki et al., 2001)

Figure 4 Comparison of initial chlorogenic acid/ caffeic acid ester oxidation products.

Initial polyphenol oxidation products are unstable electrophiles that could be stabilized by thiol conjugation (Cilliers \& Singleton, 1990), amine addition (Penalver et al., 2002; Pierpoint, 1966) or by polymerization (Cilliers \& Singleton, 1991; Namiki et al., 2001). Penalver el al. (2002) pointed out that $\alpha$-amines were weaker nucleophiles compared to thiols, which enabled thiols to trap unstable o-quinones and form thiol-ether hydroquinones. A stoichiometric relationship of thiol-quinone conjugation was reported that two molar ortho-diphenol and thiols could form 2 molar thiol-diphenol conjugates and water costing 1 molar oxygen (Penalver et al., 2002). Since cysteinyl thiol had a higher affinity to chlorogenic acid o-quinones than $\alpha$-amine groups (Lund \& Ray, 2017; Pierpoint, 1966), cysteinyl thiol was assumed to conjugate with o-quinones before $\alpha$ lysyl amine. Consequently, a competing pathway of $\varepsilon$-lysyl amine (pKa 10.5) and cysteinyl thiol (pKa 8.3) groups for chlorogenic acid quinone was postulated that $\varepsilon$-lysyl amine and cysteinyl thiol competed for initial CGA oxidation product, mono-orthoquinone. Green TBA derivatives and brown cysteinyl-CGA quinone (dimer) were formed 
as yellow electrophilic mono-ortho-quinones were stabilized by amine or thiol addition (Figure 5).

\subsubsection{Potential effects of cysteine inhibition reaction on antioxidant capacity}

Based on Danilewicz’s work (2019), at pH 8 the calculated redox potential for cystine/2 cysteine couples in the presence of iron was $-440 \mathrm{mV}$, and the calculated redox potential for quinone/catechol couple was $-836.6 \mathrm{mV}$ respectively. This signals that CGA possessed higher reducing capacity than cysteine did in alkaline solutions, so CGA might contribute to the antioxidant capacity of CGA-lysine-cysteine solutions.

Adding cysteine into alkaline CGA-lysine solutions might enhance radical scavenging capacity of the final reaction products by forming cysteinyl-CGA conjugates (Figure 5) or reducing quinones back to di-phenols. Hwang et al. (2018) reported that a mixture of cysteine and CGA thermally treated at $120^{\circ} \mathrm{C}$ for $4 \mathrm{~h}$ had significantly higher radical scavenging capacity than a heated lysine-CGA mixture subjected to the same thermal treatment. Fujimoto et al. (2013) found cysteinyl thiol had a concentrationdependent synergistic effect with methyl caffeate on preventing the formation of ethyl linoleate hydroperoxide. Less ethyl linoleate hydroperoxide was formed when cysteine and methyl caffeate were both present because the mono- and di- thiol conjugates significantly contributed to a higher radical scavenging capacity than methyl caffeate or cysteine. This enhanced antioxidant capacity is consistent with Bassil et al. (2005)’s work that 2-S-cysteinylcaffeic acid (2-CCA), the primary reaction product of caffeic acid and cysteine, had a higher radical scavenging capacity than caffeic acid or cysteine alone. Caffeic acid and 2-CCA have been shown to reduce metmyoglobin (MetMb) to oxymyoglobin $\left(\mathrm{MbO}_{2}\right.$ ) (Miura, Inai, Honda, Masuda, \& Masuda, 2014). At pH 7.4, 
cysteine alone reduced MetMb twice as much as caffeic acid did while the mixture of cysteine and caffeic acid reduced 39\% more MetMb to $\mathrm{MbO}_{2}$ than cysteine did due to formation of 2-CCA. The reduction potential order of these antioxidants was 2-CCA $<$ cysteine $<$ caffeic acid. Oxidized 2-CCA did not oxidize $\mathrm{MbO}_{2}$ back to MetMb as caffeic acid quinone would (Miura et al., 2014), indicating that caffeic acid quinone had a higher reduction potential than oxidized thiol-substituted caffeic acid. In addition, Kuijpers et al. (2012) found that CGA had a 15\% higher relative oxygen consumption than a mixture of CGA and cysteine did when they were treated with the same concentration of tyrosinase at pH 6.5 without pre-incubation. When the mixture of CGA and cysteine mixtures was pre-incubated for 15 mins at $25^{\circ} \mathrm{C}$, the relative oxygen consumption of the mixture was more than 35\% lower than that of not pre-incubated CGA. This indicated that 2-Scysteinyl-CGA might have a higher reducing capacity as 2-S-cysteinyl-CGA did not inhibit mushroom tyrosinase (Kuijpers et al., 2012). Consequently, adding cysteine into a lysine-CGA mixture is expected to enhance antioxidant capacity contributed due to formation of cysteinyl-CGA conjugates, which could possibly have a higher antioxidant capacity than CGA given the similarities in structure between caffeic acid and CGA (Bassil et al., 2005). 


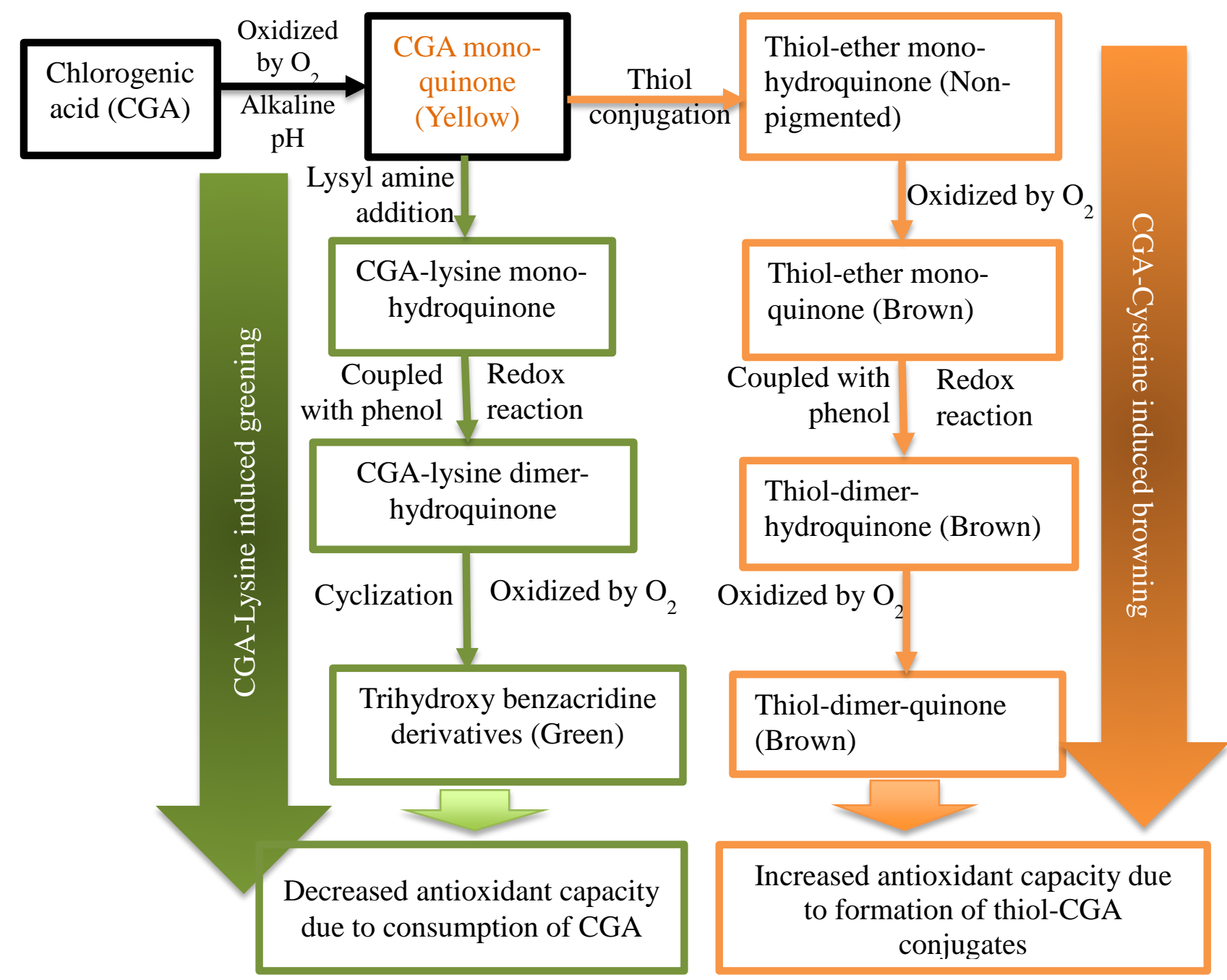

Figure 5 Postulated mechanism and effects on antioxidant capacity of $\varepsilon$-lysyl amine and cysteinyl thiol groups competing for chlorogenic acid o-quinone, which induced greening and browning respectively in aqueous solution at alkaline $\mathrm{pH}$ (cysteinyl thiol $\mathrm{pKa}$ ), adapted from Yabuta et al. (2001), Pierpoint (1966) and Penalver et al. (2002).

\subsection{Rationale and Significance}

Greening in CGA-lysine solutions and alkali baked foods is attributed to formation of green TBA derivatives at alkaline pH (Liang \& Were, 2018b). CGA quinones and primary amine groups are precursors for green pigments with TBA chromophore structures and brown melanoidins. Amino acids with a nucleophilic side group: cysteine, serine and threonine, do not covalently bond with CGA quinones to form amine-CGA conjugates and subsequently to form green pigments under alkaline 
conditions. Between two commonly used dough conditioners, glutathione and cysteine, Kuijpers (2013) found that L-cysteine had a higher tendency to form adducts with oquinones, indicating L-cysteine as a potentially more effective greening inhibitor than glutathione. Furthermore, a heated CGA-cysteine mixture produces thiol-quinone derivatives with significantly higher radical scavenging activity compared with a heated CGA-lysine mixture (Hwang et al., 2018). However, whether L-cysteine, a U.S. FDAapproved GRAS dough conditioner, de-greens CGA quinone-amine induced greening under alkaline conditions is unknown. In addition, Lund and Ray (2017) and Pierpoint (1966) reported that cysteinyl thiol had a higher affinity for chlorogenic acid o-quinones $\alpha$-amine groups, so cysteinyl thiol was assumed to conjugate with o-quinones before $\alpha$ lysyl amine. However, affinities of $\varepsilon$-lysyl amine (pKa 10.5) and cysteinyl thiol (pKa 8.3) groups for chlorogenic acid quinone at alkaline $\mathrm{pH}$ were not reported in any studies.

The overall research goal was to investigate how different concentrations of Lcysteine react in CGA-lysine solutions, and its subsequent effects on antioxidant reducing capacity. This overall goal was accomplished through the following aims:

I. Investigate the effect of 5 cysteine concentrations alone and combined with different alkaline $\mathrm{pH}$ levels $(7.75,8.0$ and 9.0) on formation of green TBA derivatives in CGA-lysine solutions. It was hypothesized that L-cysteine inhibits greening in a concentration dependent manner by forming colorless cysteineCGA conjugates (Bongartz et al., 2016; Schilling et al., 2008). Greater antigreening of the same cysteine concentration was expected at higher $\mathrm{pH}$ as formation of cysteine-CGA conjugates is promoted by higher $\mathrm{pH}$ (Rawel \& Rohn, 2010). 
II. Elucidate the mechanism of how L-cysteine reacts with CGA in the presence of L-lysine, a reactive amino acid that forms TBA chromophores, in buffered CGA: lysine: cysteine solutions (2.55 mM: $5.09 \mathrm{mM}: 0-5.091 \mathrm{mM})$ at $\mathrm{pH}$ 7.75, 8.0 and 9.0. It was hypothesized that $\varepsilon$-lysyl amine and cysteinyl thiol competed for initial CGA oxidation products, mono-ortho-quinone; in addition, green TBA derivatives and brown cysteinyl-CGA quinone (dimer) were formed as yellow electrophilic mono-ortho-CGA quinones were stabilized by amine or thiol addition (Cilliers \& Singleton, 1990; Penalver et al., 2002).

III. Determine the effect of cysteinyl-CGA conjugates on antioxidant capacity. It was hypothesized that adding cysteine into alkaline CGA-lysine solutions enhanced radical scavenging capacity of the final reaction products by forming cysteinyl-CGA conjugates (Bassil et al., 2005; Fujimoto et al., 2013).

Mechanism of how L-cysteine reacts with CGA in the presence of L-lysine forming chromophores is significant for filling the knowledge gap of how thiol and $\varepsilon$ amine groups, two highly reactive amino acid side chains compete for electrophilic oquinones under alkaline conditions.

This information can be applicable to develop chlorogenic acid-containing sunflower protein products in the future, which are recognized as one of the top six plantbased protein ingredients (Ferrer, 2019). The results will provide an innovative method of high yield minimally green protein from sunflower meal with enhanced antioxidant capacity but no negative influence on sustainability. For chlorogenic acid-rich baked goods, using cysteine could be a potential anti-greening strategy with enhanced antioxidant capacity. 


\section{Materials and Methods}

\subsection{Experimental design}

L-cysteine effects were first investigated in unbuffered pH 9.0 CGA: lysine (Lys): cysteine (Cys) solutions, and then in pH 7.75, 8.0 and 9.0 CGA: lysine: cysteine solutions prepared with phosphate buffers, which were incubated for $48 \mathrm{~h}$ at ambient temperature. A factorial design for cysteine effects on chlorogenic acid quinone-lysine induced greening over $48 \mathrm{~h}$ was implemented with 6 cysteine concentrations (0 to $5.091 \mathrm{mM}$ ), and $3 \mathrm{pH}$ (buffered $\mathrm{pH}$ 7.75, 8.0, 9.0, and unbuffered $\mathrm{pH} 9.0$ ) and 10 or 3 time points (10 for color intensities and 3 for antioxidant capacity as variables. Color intensities and $\mathrm{pH}$ were monitored hourly between 1 to $8 \mathrm{~h}$ and after 24 and $48 \mathrm{~h}$. Antioxidant capacity assessments by FCRC (Folin-Ciocalteu reagent reducing capacity) and TEAC (Trolox Equivalents Antioxidant Capacity) assays, CGA-amino acid conjugates quantification by HPLC and identification by LC/MS were conducted after $48 \mathrm{~h}$ of incubation (Figure 6). Forty-eight hour incubated solutions were aliquoted in $1.5 \mathrm{~mL}$ Eppendorf tubes and stored in $-80{ }^{\circ} \mathrm{C}$ freezer for further analysis. Three replicates were prepared for each treatment. Measurements were repeated three times for each replicate in all analysis. 


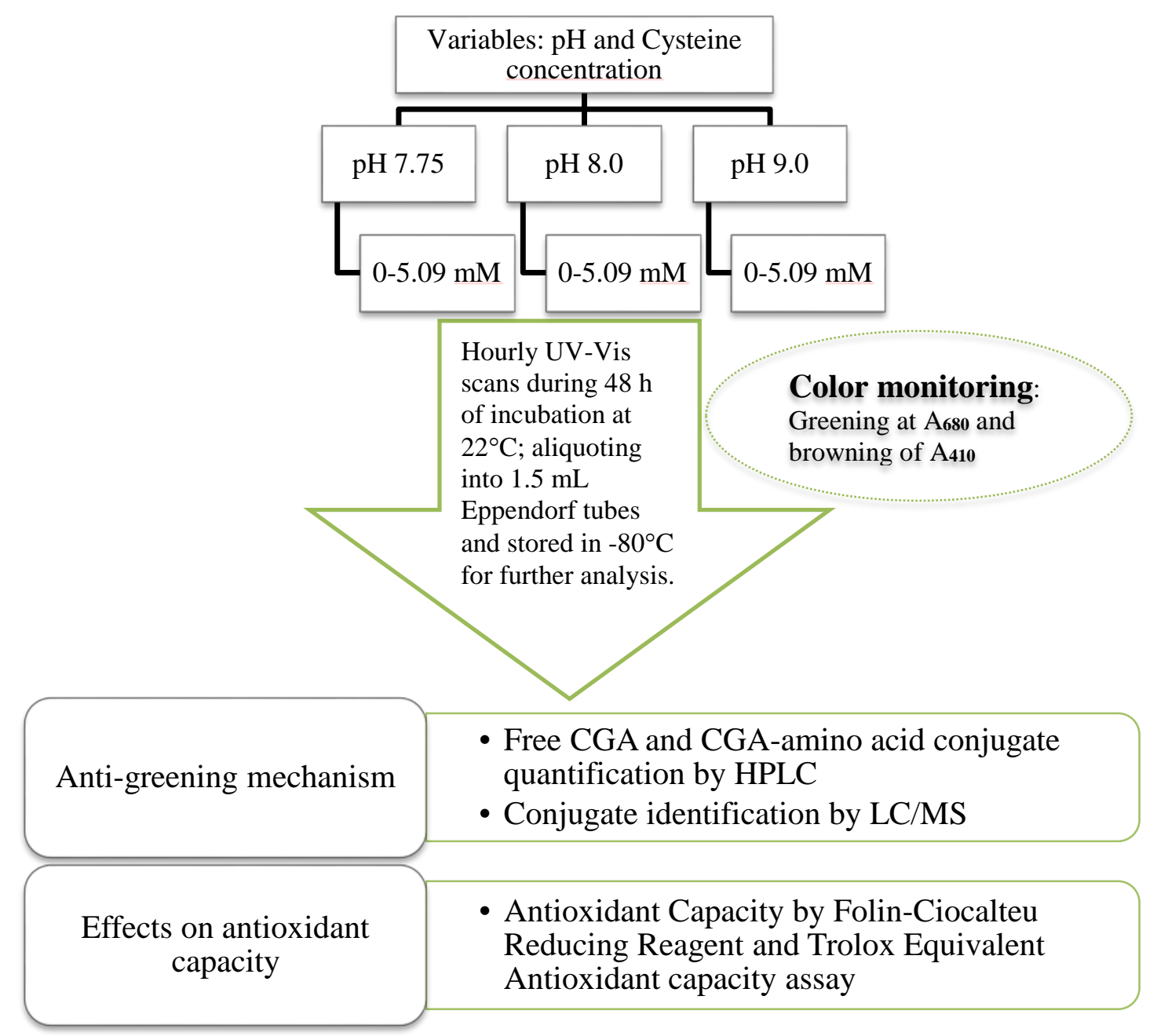

Figure 6 Experimental design for cysteine effects on color intensities, reaction products and antioxidant capacity as a function of $\mathrm{pH}$ and cysteine concentration in chlorogenic acid: L-lysine: L-cysteine solutions.

\subsection{Solution and buffer preparation and UV-Vis scans of model solutions}

Anhydrous L-cysteine hydrochloride of 98\% purity, purchased from Acros

Organics (New Jersey, USA), chlorogenic acid (>95\%; TLC) and L-lysine (>98\%; TLC) purchased from Sigma-Aldrich (St. Louis, Missouri, USA), were dissolved in pH 7.75, 8.0 and 9.0 phosphate buffers, and deionized water to prepare L-cysteine, CGA, and Llysine buffered and unbuffered stock solutions. Phosphate buffers at $\mathrm{pH}$ 7.75, 8.0 and 9.0 
were prepared $24 \mathrm{~h}$ in advance. Buffers were adjusted to corresponding $\mathrm{pH}$ with $6 \mathrm{~N} \mathrm{HCl}$ or $6 \mathrm{~N} \mathrm{NaOH}$ solutions initially, and again 90 mins before CGA-amino acid solutions were prepared. Unbuffered stock CGA, L-lysine and L-cysteine solutions were dissolved in DI water before adjusted to $\mathrm{pH} 8$ and 9 using $6 \mathrm{~N} \mathrm{HCl}$ or $\mathrm{NaOH}$ solutions. One hundred milliliters of 5.6 mM CGA, $11.2 \mathrm{mM}$ lysine solutions, and $20 \mathrm{~mL} 1980$ L-cysteine solutions were prepared by dissolving $0.1984 \mathrm{~g}$ chlorogenic acid, $0.1637 \mathrm{~g}$ L-lysine and $0.0396 \mathrm{~g}$ L- cysteine $\bullet \mathrm{HCl}$ solids respectively into deionized (DI) water or phosphate buffers. Two milliliters of buffered 5.6 mM CGA, $11.2 \mathrm{mM}$ lysine solutions with 0.4 milliliter of $\mathrm{pH} 8.0$ or 9.0 phosphate buffer were prepared as negative controls.

Buffered and unbuffered cysteine solutions were diluted with phosphate buffer and DI water respectively to achieve a final cysteine concentration of 0 to $5.09 \mathrm{mM}$. For each concentration of L-cysteine, triplicates of each reaction mixture were prepared at a ratio of 1:1:0.2 CGA: lysine: cysteine solutions for a final concentration of $2.55 \mathrm{mM}$ CGA: 5.09 mM Lys: 0-5.09 mM Cys. L-cysteine and L-lysine solutions were mixed first, and reaction starting time points were recorded after adding CGA solutions into the mixture.

To investigate the effect of $\mathrm{pH}$ on chlorogenic acid-amino acid non-enzymatic color reactions, buffered and unbuffered CGA: lysine: cysteine solutions were monitored at $410 \mathrm{~nm}$ for browning and $680 \mathrm{~nm}$ for greening in a flat bottom Falcon 96 microplate with FLUOstar Omega Microplate Reader (BMG Labtech, Ortenberg, Germany) hourly from 1 to 8 , after 24 and $48 \mathrm{~h}$ of incubation at ambient temperature $\left(\sim 22^{\circ} \mathrm{C}\right)$. $\mathrm{pH}$ by Vernier pH sensor (Vernier Software and Technology, Beaverton, OR) was measured after UV-Vis scans at each time point. 


\subsection{Folin-Ciocalteu Reagent reducing and Trolox equivalent antioxidant capacity (TEAC) assays of CGA: lysine: cysteine solutions}

Folin-Ciocalteu Reagent reducing capacity of CGA: Lys: Cys solutions was performed as outlined by Yu, Nanguet and Beta (2013). A Gallic acid standard curve was used to measure the Reducing capacity of reaction solutions, recorded as mg gallic acid equivalents per milliliter of solution.

Trolox equivalent antioxidant capacity (TEAC) assay by measuring ABTS+ radical scavenging activity was slightly modified from Hwang et al. (2018). The stock radical solution was prepared by mixing $2.45 \mathrm{mM}$ potassium persulfate with $7 \mathrm{mM}$ ABTS + in the dark for $24 \mathrm{~h}$ at ambient temperature $\left(\sim 22^{\circ} \mathrm{C}\right)$. Twenty microliters of CGA: Lys: Cys solutions and $180 \mu \mathrm{L}$ ABTS+ solution was reacted in 96-well Falcon plate. Absorbance at $734 \mathrm{~nm}$ was taken after $10 \mathrm{~min}$ on a microplate reader (FLUOstar Omega Microplate Reader, BMG Labtech, Ortenberg, Germany). Trolox was used as the positive control, and results were recorded as millimoles of Trolox per milliliter of solution.

\subsection{Unreacted CGA and CGA conjugates quantification by HPLC}

Twenty percent diluted CGA: Lys: Cys solutions with HPLC water were used for HPLC analysis. Unreacted CGA and cysteinyl-CGA conjugates quantifications were carried out on an Agilent 1100 series HPLC (Agilent Technologies, Inc. Santa Clara, CA, USA) with G1315B diode array detector monitoring at $294 \mathrm{~nm}, 320 \mathrm{~nm}, 450 \mathrm{~nm}$, and 680 nm. A Phenomenex ${ }^{\circledR}$ Luna Omega $5 \mu$ C18 (2) $100 \AA \AA(150 \times 2$ mm, $1.5 \mu$ m particle size) column was used with HPLC water acidified with $0.1 \%(\mathrm{v} / \mathrm{v})$ formic acid and acetonitrile acidified with $0.1 \%(\mathrm{v} / \mathrm{v})$ formic acid were as mobile phase A and B. The gradient used 
was 0 min, $0 \%$ B; 1.5 min, 22.0\% B; 4.5 min, 26.0\% B; 6 min, 28.0\% B; 7-7.1 min, isocratic on $30 \% \mathrm{~B}$; 7.1-8 min, linear gradient from $30 \%$ to $100 \% \mathrm{~B}$; $8.0-10 \mathrm{~min}$, isocratic on $100 \%$; $10-11 \mathrm{~min}$, isocratic on $0 \% \mathrm{~B}$, at $1.0 \mathrm{~mL} / \mathrm{min}$ flow rate with the oven temperature controlled at $30^{\circ} \mathrm{C}$.

\subsection{Identification of CGA-cys adducts and TBA derivatives in solution model by LC-MS}

Chlorogenic acid, lysine and cysteine standards prepared in $\mathrm{pH} 8$ and 9 were diluted to $10 \%$ for LC-MS analysis. Phenolic-amino acid adducts were determined with Dionex UltiMate3000 UHPLC equipped with an autosampler and diode array detector. A Bruker Impact IITM UHR-QTOF MS (Bruker, Billerica, MA, USA) was used. Temperature was set at $30{ }^{\circ} \mathrm{C}$, monitored at wavelengths of 280, 320, 450 and $680 \mathrm{~nm}$ and a flow rate of $0.3 \mathrm{~mL} / \mathrm{min}$. Mobile phase A was $0.1 \%$ formic acid in HPLC-grade water. Gradient used was 0 min, $0 \%$ B; $1-1.5$ mins, linear gradient from $0 \%$ to $15 \% \mathrm{~B}$; 1.5 - 4.5 mins, linear gradient from $15 \%$ to $30 \%$; 4.5 to 6.0 mins $30 \%$ to $45 \%$ B; 6 - 8 mins, linear gradient $45 \%$ to $100 \%$ B; 8 - 9 mins isocratic on $100 \%$ and 9 -10 mins $0 \%$ of mobile phase B (0.1\% formic acid in HPLC-grade acetonitrile).

Mass spectrometry analysis was carried out in the positive mode on a HP 1100MSD series (Agilent Technologies) equipped with an ESI interface and LC/ MSD ChemStation software (Agilent Technologies, 2002). The MS detector was in API-ES, positive polarity mode, drying gas flow was $4.0 \mathrm{~L} / \mathrm{min}$ at $180^{\circ} \mathrm{C}$, nebulizer pressure was 35 psi, capillary voltage was $4500 \mathrm{~V}$, and the mass spectra of eluate positive ions ranged from m/z 50 to 1200. 


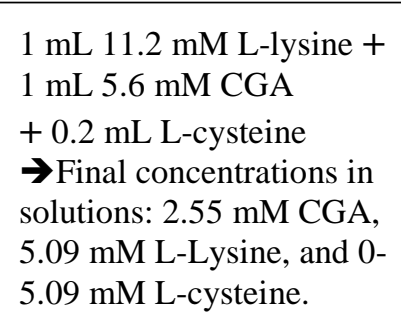

Primary expected

$[\mathrm{M}+\mathrm{H}]^{-}$ion fragments from LC/MS/MS:

$\Rightarrow \mathrm{m} / \mathrm{z} 700$, TBA core structure;

$\Rightarrow \mathrm{m} / \mathrm{z}$ 829, CGA quinone-lys adduct; $\Rightarrow \mathrm{m} / \mathrm{z} 473$, CGA-cys adduct (2'-S-cysteinyl5-O-caffeolquinic acid).

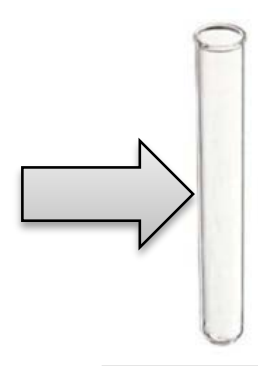

Incubation for 48 hours at $22^{\circ} \mathrm{C}$

\section{LC/MS/MS}

-Monitoring at $\mathbf{2 8 0} \mathbf{~ n m}$ for benzacridine derivatives (Iacomino et al., 2017), $320 \mathbf{~ n m}$ for hydroxyl cinnamic acid derivatives (Kuijpers et al., 2012), $450 \mathbf{~ n m}$ and $680 \mathrm{~nm}$ for TBA isomers (Bongartz et al., 2016)

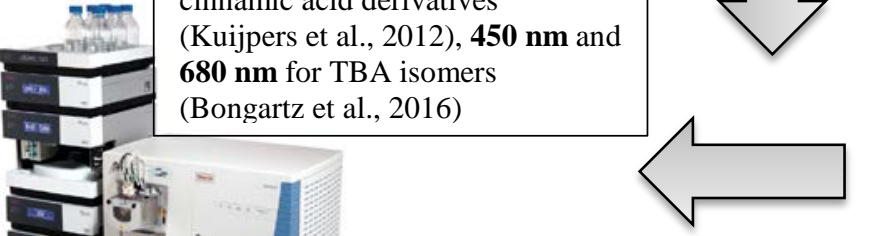

Diluted to $5 \%(\mathrm{v} / \mathrm{v})$ with HPLC grade water.

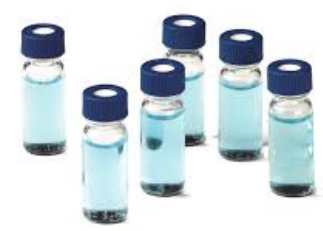

Figure 7 Conjugation analysis on 1: 1: 0.22 .55 mM CGA: 5.09 mM L-Lysine: 0-5.09 mM L-cysteine solutions.

\subsection{Statistical analysis}

A complete 2 × 6 × 10 factorial design for color intensities and a $2 \times 6 \times 3$ factorial design for antioxidant capacity were implemented with $2 \mathrm{pH}$ (8 and 9), 6 cysteine concentrations $(0,1.02,1.27,2.55,3.82$ and $5.09 \mathrm{mM})$ and 3 or 10 different incubation time points (hourly for the first $8 \mathrm{hrs}$ and after 24 and $48 \mathrm{~h}$ ), and each of 36 treatment (Figure 6) was prepared in triplicates. Three measurements were taken for each sample.

The effect of cysteine concentration, $\mathrm{pH}$, incubation time and their interactions were evaluated in a three-way factorial Analysis of Variance (ANOVA): two fixed effects (cysteine concentration and $\mathrm{pH}$ ) as variables were repeated at 10 different incubation time points. Duncan’s Multiple Range test was followed to determine the 
differences between browning $/ \mathrm{A}_{410 \mathrm{~nm}}$, greening $/ \mathrm{A}_{680 \mathrm{~nm}}$, and antioxidant capacity) and three-way interactions of independent variables (pH and cysteine concentrations). Linear regression analysis to model the relationship between color intensities ( $\mathrm{A}_{410} \mathrm{~nm}$ and $\mathrm{A}_{680}$ $\mathrm{nm}$ ) and cysteine concentration (0 to $5.091 \mathrm{mM}$ ) at 2 different $\mathrm{pH}(\mathrm{pH} 8$ and 9) and 10 different incubation time points were assessed with Statistical Analysis Software 9.3 (SAS, 2014) at a significance level $\alpha=0.05$.

\section{Results and Discussion Color intensities in unbuffered CGA: Lys: Cys solutions}

Data from the first experiment in unbuffered pH 8 and 9 CGA: Lys: Cys solutions (Figure 8A\&C) showed browning decreased after incubation attributed to consumption of brown CGA quinones to form green TBA derivatives (Figure 8B\&D). Browning was higher in alkaline CGA: Lys: Cys solutions with higher cysteine concentration after $48 \mathrm{~h}$ incubation. 
A. Browning intensity in unbuffered $\mathrm{pH} 8$ CGA: Lys: Cys solutions

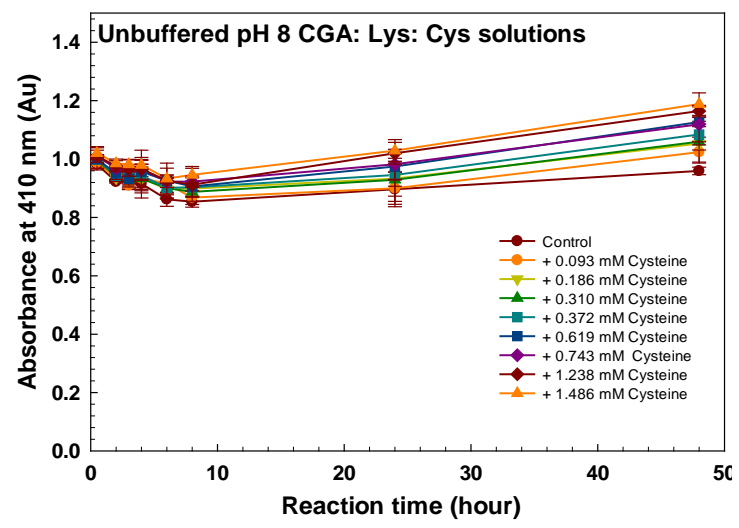

C. Browning intensity in unbuffered $\mathrm{pH} 9$ CGA: Lys: Cys solutions ( $\mathrm{pH}_{48 \mathrm{~h}}$ was from 7.88 to 8.09 due to oxidation and deprotonation)

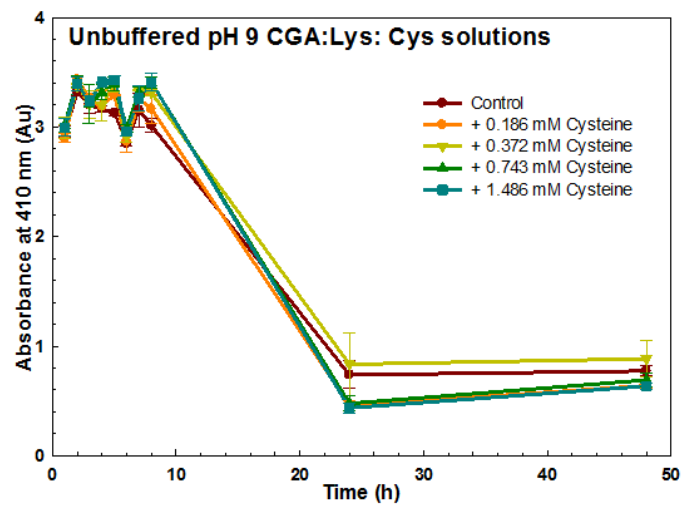

B. Greening intensity in unbuffered $\mathrm{pH} 8$ CGA: Lys: Cys solutions

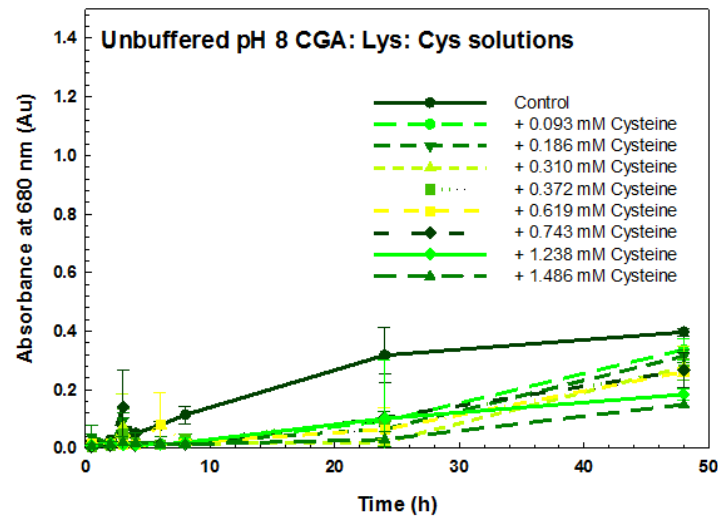

D. Greening intensity in unbuffered $\mathrm{pH} 9$ CGA: Lys: Cys solutions $\left(\mathrm{pH}_{48 \mathrm{~h}}\right.$ was from 7.88 to 8.09 due to oxidation and deprotonation)

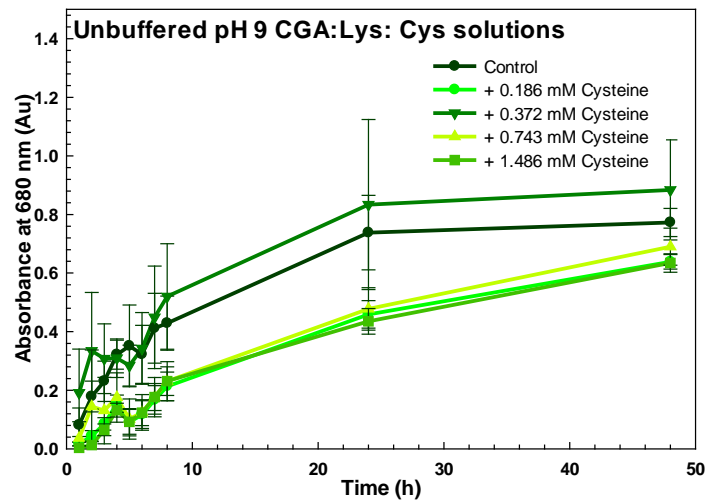

Figure 8 Browning measured by absorbance at $410 \mathrm{~nm}(\mathrm{~A}, \mathrm{C})$ and greening measured by absorbance at $680 \mathrm{~nm}(\mathrm{~B}, \mathrm{D})$ in unbuffered $\mathrm{pH} 8$ and 9 CGA: lysine: cysteine solutions as a function of reaction time for $48 \mathrm{~h}$ when cysteine increased from 0 to $1.486 \mathrm{mM}$.

A strong negative correlation was found between cysteine concentration and greening while a positive correlation was found for browning. Since covalent bond formations for TBA and amino acid-CGA conjugates lowered $\mathrm{pH}$ of all the unbuffered solutions, $\mathrm{pH}$ was a confounding factor (Table 3) because the greening inhibition was not all attributed to addition of cysteine, either forming colorless conjugates or reducing CGA o-quinones back to CGA diphenols. 
Table 3 pH of unbuffered pH 9 CGA: Lys: Cys solutions over 48 h incubation at ambient temperature.

\begin{tabular}{cccccc}
\hline Time (h) & $\mathbf{0 ~} \mathbf{~ m M}$ & $\mathbf{0 . 1 8 6} \mathbf{~ m M}$ & $\mathbf{0 . 3 7 2} \mathbf{~ m M}$ & $\mathbf{0 . 7 4 3} \mathbf{~ m M}$ & $\mathbf{1 . 4 8 6} \mathbf{~ m M}$ \\
\hline $\mathbf{0}$ & $9.00 \pm 0.002$ & $9.00 \pm 0.012$ & $9.00 \pm 0.015$ & $9.00 \pm 0.008$ & $9.00 \pm 0.027$ \\
$\mathbf{1 . 5}$ & $8.67 \pm 0.025$ & $8.53 \pm 0.061$ & $8.50 \pm 0.046$ & $8.53 \pm 0.006$ & $8.45 \pm 0.006$ \\
$\mathbf{3 . 5}$ & $8.28 \pm 0.031$ & $8.15 \pm 0.006$ & $8.17 \pm 0.015$ & $8.18 \pm 0.015$ & $8.23 \pm 0.017$ \\
$\mathbf{5}$ & $8.47 \pm 0.049$ & $8.46 \pm 0.041$ & $8.49 \pm 0.029$ & $8.43 \pm 0.289$ & $8.42 \pm 0.040$ \\
$\mathbf{6}$ & $8.77 \pm 0.075$ & $8.74 \pm 0.021$ & $8.68 \pm 0.061$ & $8.61 \pm 0.070$ & $8.43 \pm 0.095$ \\
$\mathbf{7}$ & $8.20 \pm 0.062$ & $8.28 \pm 0.029$ & $8.27 \pm 0.036$ & $8.36 \pm 0.006$ & $8.37 \pm 0.015$ \\
$\mathbf{8}$ & $8.31 \pm 0.055$ & $8.26 \pm 0.025$ & $8.29 \pm 0.015$ & $8.31 \pm 0.006$ & $8.33 \pm 0.006$ \\
$\mathbf{2 4}$ & $8.28 \pm 0.115$ & $8.30 \pm 0.032$ & $8.29 \pm 0.031$ & $8.26 \pm 0.015$ & $8.21 \pm 0.031$ \\
$\mathbf{4 8}$ & $8.09 \pm 0.049$ & $8.03 \pm 0.012$ & $7.96 \pm 0.025$ & $7.89 \pm 0.050$ & $7.88 \pm 0.031$ \\
$\mathbf{\%} \mathbf{\%} \mathbf{1}$ & $-10.15 \%$ & $-10.81 \%$ & $-11.52 \%$ & $-12.30 \%$ & $-12.48 \%$ \\
reduction & & & & & \\
\hline
\end{tabular}

Solutions with higher cysteine concentrations had a lower $\mathrm{pH}$. Since both lower $\mathrm{pH}$ and higher cysteine could decrease greening in alkaline CGA: Lys: Cys solutions, preparing buffered CGA: Lys: Cys solutions was necessary to rule out $\mathrm{pH}$ change with added cys as a confounding factor in subsequent solutions.

\subsection{Effects of cysteine concentration on color intensities}

Cysteine competitive inhibition of greening (A680 nm, Figure 9B) was concentration-dependent after $24 \mathrm{~h}$ at all tested $\mathrm{pH}\left(\mathrm{r}_{\mathrm{pH} 7.75}=-0.5053, \mathrm{r}_{\mathrm{pH} 8}=-0.7041, \mathrm{r}_{\mathrm{pH} 9}\right.$ $=-0.8791$ ) when cysteine concentration was increased from 0 to $1.486 \mathrm{mM}$. Browning (A410 nm) positively correlated with cysteine concentration at pH 7.75-9 after $24 \mathrm{~h}$ incubation $\left(\mathrm{r}_{\mathrm{pH} 9}=0.91171, \mathrm{r}_{\mathrm{pH} 8}=0.61524, \mathrm{r}_{\mathrm{pH} 7.75}=0.56401\right)$. Initial browning $\left(\mathrm{A}_{410 \mathrm{~nm}}\right)$ in CGA: Lys: 0-1.486 mM Cys solutions was primarily due to formation of brown yellow CGA o-quinones. Higher browning was observed with higher cysteine concentration after incubation because more amine precursors of melanoidins were present in higher cysteine solutions (Figure 9). 
A. Browning intensity in $\mathrm{pH} 7.75,8$ and 9 buffered CGA: lysine: cysteine solutions

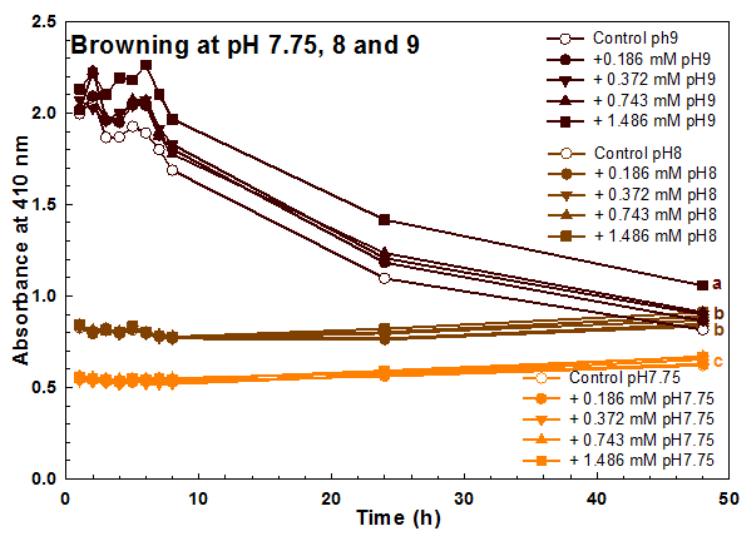

B. Greening intensity in $\mathrm{pH} 7.75,8$ and 9 buffered CGA: lysine: cysteine solutions

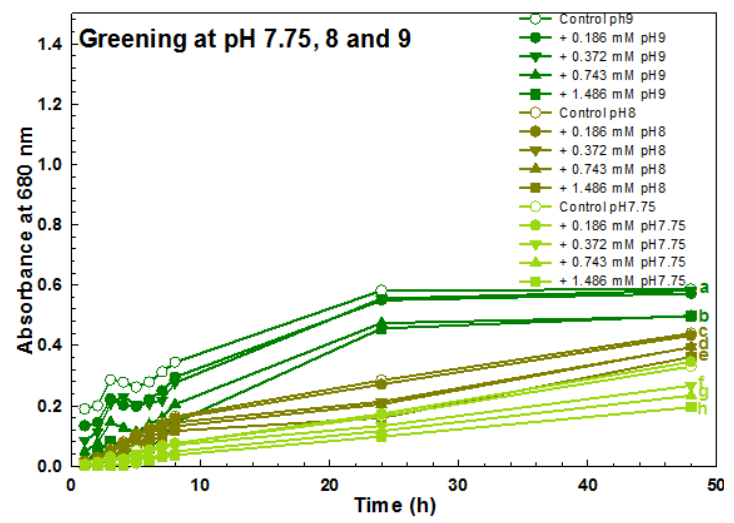

Figure 9 Browning measured by absorbance at $410 \mathrm{~nm}$ (A) and greening measured by absorbance at $680 \mathrm{~nm}(\mathrm{~B})$ in $\mathrm{pH}$ 7.75, 8 and 9 buffered CGA: lysine: cysteine solutions as a function of reaction time for $48 \mathrm{~h}$ when cysteine increased from 0 to $1.486 \mathrm{mM}$. The data presented were means of 3 replicates. Means with the same letter after $48 \mathrm{~h}$ were not significantly different.

As greening $\left(\mathrm{A}_{680 \mathrm{~nm}}\right)$ occurred at $\mathrm{pH} 7.75,8.0$ and 9.0 at highest cysteine concentration $1.486 \mathrm{mM}$ (Figure 9B), cysteine concentration range was then increased from 0 to $5.09 \mathrm{mM}$ in subsequent experiments, and pH7.75 was eliminated because hyperchromic shifts at $410 \mathrm{~nm}$ and $680 \mathrm{~nm}$ followed a similar trend throughout incubation at $\mathrm{pH} 7.75$ and 8.0 (Figure 9). When cysteine concentration was increased to $5.09 \mathrm{mM}$, no visible greening was observed at both $\mathrm{pH} 8.0$ and 9.0 after 24 h incubation (Figure 10 F\&L). Therefore, buffered pH 8.0 and 9.0, 2.55 mM CGA: 5.09 mM Lys: 05.09 mM Cys solutions were studied from 0 to $5.09 \mathrm{mM}$ cysteine.

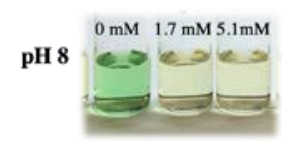

A. $1 \mathrm{~h}$

pH 9

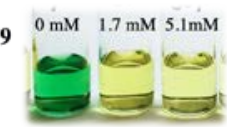

G. $1 \mathrm{~h}$

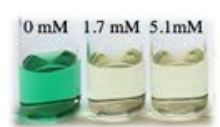

B. $2.5 \mathrm{~h}$

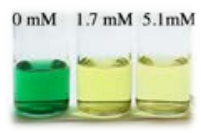

H. $2.5 \mathrm{~h}$

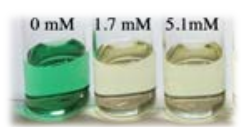

C. $4 \mathrm{~h}$

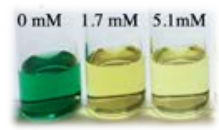

I. $4 \mathrm{~h}$

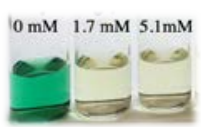

D. $5.5 \mathrm{~h}$

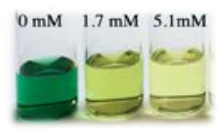

J. $5.5 \mathrm{~h}$

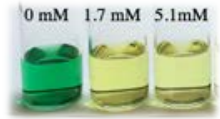

E. $7 \mathrm{~h}$

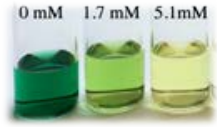

K. $7 \mathrm{~h}$

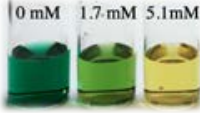

F. $24 \mathrm{~h}$

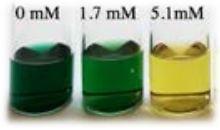

L. $24 \mathrm{~h}$

Figure 10 pH 8 (A to F) and pH 9 (G to L) buffered CGA: Lys: Cys solutions with $0 \mathrm{mM}$ (control), $1.7 \mathrm{mM}$ and $5.1 \mathrm{mM}$ cysteine incubated for $24 \mathrm{~h}$ at ambient temperature. 
In 2.55 mM CGA: 5.09 mM Lys: 0-5.09 mM Cys solutions, a hypochromic shift at $680 \mathrm{~nm}$ was observed as cysteine increased at both $\mathrm{pH} 8$ and 9 (Figure 11C\&D). Stronger negative correlations between cysteine concentration and greening $\left(\mathrm{A}_{680} \mathrm{~nm}\right)$ were observed as solutions were incubated over time, which confirmed that cysteine inhibited CGA quinone-lysine greening in a concentration dependent manner. After 48 h, greening occurred at lower cysteine concentration while no visible greening was observed when cysteine was $5.09 \mathrm{mM}$ at pH 8 and 9 (Figure $10 \mathrm{~F} \& \mathrm{~L}$; Figure $11 \mathrm{C} \& \mathrm{D}$ ). Cysteine of $5.09 \mathrm{mM}$ was the same concentration as lysine in non-green solutions, indicating that cysteinyl thiols (-SH) had a higher affinity than $\varepsilon$-lysyl amine for CGA $o$ quinones for nucleophilic conjugation. This was similar to findings by Lund and Ray (2017) and Pierpoint (1966) who found that cysteinyl thiols have a higher affinity to CGA $o$-quinones than $\alpha$-amines from amino acids. Greening was observed at cysteine concentrations less than $5.09 \mathrm{mM}$ because more lysine was present. When cysteine concentrations were less than that of lysine, lack of greening was temporary as CGA quinones that were reduced to CGA hydroquinones could regenerate CGA hydroquinones undergoing further oxidative reactions to form green TBA or yellow TBA derivatives with time. The 830 Da yellow TBA derivatives (Figure 12) were not oxidized to green TBAs in the first $24 \mathrm{~h}$.

Initial browning $\left(\mathrm{A}_{410 \mathrm{~nm}}\right)$ in CGA: Lys: Cys solutions were primarily because CGA was oxidized to brown CGA quinones in alkaline $\mathrm{pH}$ solutions. Hypochromic shift at $410 \mathrm{~nm}$ over $48 \mathrm{~h}$ incubation was due to consumption of brown CGA quinones. Besides forming green pigments with amines, CGA quinones can also be consumed by cysteine to form colorless cysteinyl-CGA conjugates, or cysteine could reduce CGA 
quinones to colorless CGA diphenols, leading to lowered browning. From 24 to $48 \mathrm{~h}$, hyperchromic shift at $410 \mathrm{~nm}$ was observed in all treatments except controls at $\mathrm{pH} 8$ and 9 because regenerated CGA diphenols were oxidized again to brown CGA quinones. After 48 h, higher browning was observed with higher cysteine concentration, which might be due to more amines present in high cysteine solutions (Figure 11 A\&B). Amines can react with quinones to form imines, which can then subsequently undergo Maillard reaction to form melanoidins (Lund et al., 2011; Lund \& Ray, 2017).

Cysteine inhibited CGA quinone induced greening in buffered pH 8 and 9 CGA: Lys: $5.09 \mathrm{mM}$ Cys solutions in a concentration dependent manner by two different pathways. One is by forming colorless cysteinyl-CGA conjugates (Figure 12), and the other is by reducing quinones to CGA di-phenols (Figure 13) to limit availability of CGA quinones, one of the precursors of green trihydroxy benzacridine. At pH 8 and 9, CGA quinones were first attacked at the most electrophilic position 2 of the phenol ring by nucleophiles (cysteinyl thiols and $\varepsilon$-lysyl amines) to form colorless cysteinyl-CGA conjugates (compound 3\&4, Table 6). Formation of di-cysteinyl CGA conjugates (compound 4) was reported by Pierpoint (1966) and Schilling et al. (2008), when CGA oquinones was substituted at position 5 of the phenol ring when excess thiols were present because the $5^{\text {th }}$ position was the second most electrophilic position of a CGA $o$-quinone. When CGA reacted with lysine, the $2^{\text {nd }}$ position of CGA quinone could also be nucleophilically attacked by lysyl amines, leading to formation of green trihydroxy benzacridine (Figure 12).

A. Browning in $\mathrm{pH} 8$ buffered CGA: lysine: cysteine solutions incubated at ambient
B. Browning in pH 9 buffered CGA: lysine: cysteine solutions incubated at ambient 
temperature for $48 \mathrm{~h}$

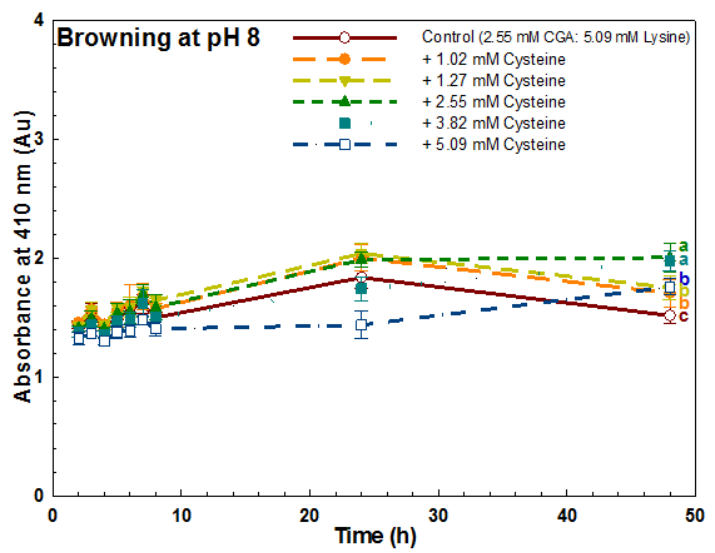

C. Greening in pH 8 buffered CGA: lysine: cysteine solutions incubated at ambient temperature for $48 \mathrm{~h}$

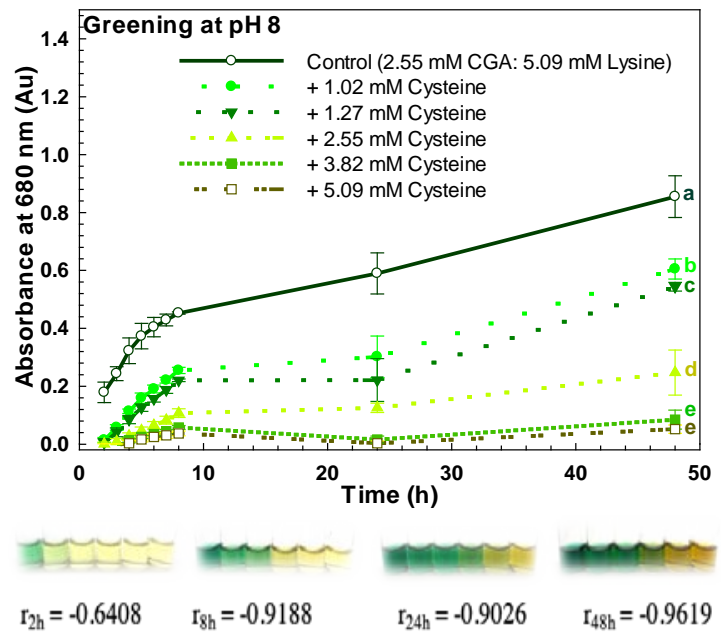

temperature for $48 \mathrm{~h}$

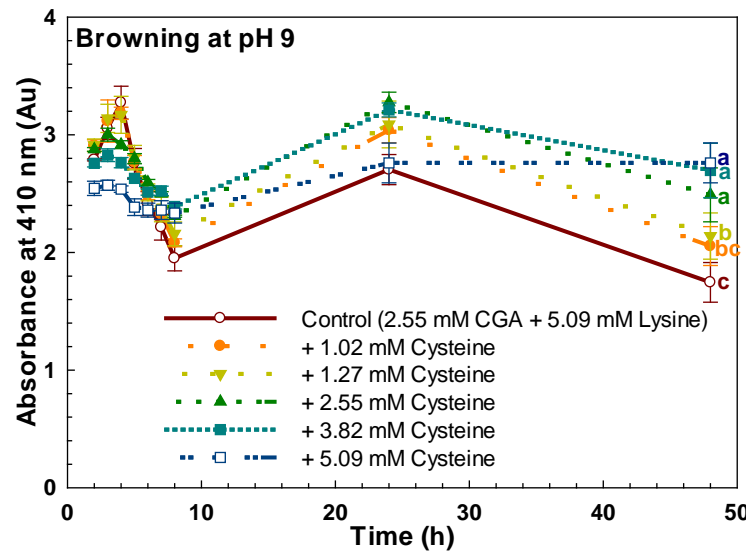

D. Greening in pH 9 buffered CGA: lysine: cysteine solutions incubated at ambient temperature for $48 \mathrm{~h}$

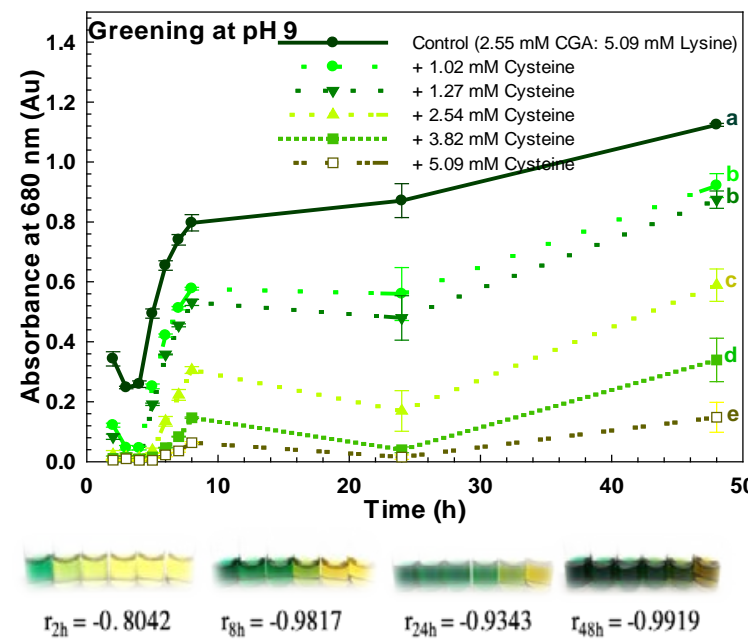

Figure 11 Browning measured by absorbance at $410 \mathrm{~nm}$ (A, B), greening measured by absorbance at $680 \mathrm{~nm}(\mathrm{C}, \mathrm{D})$ and photos representing visual appearance with corresponding correlation coefficients between -0.6408 and -0.9919 of $\mathrm{pH} 8$ and 9 buffered chlorogenic acid (CGA): lysine: cysteine solutions incubated at ambient temperature when cysteine increased from 0 to $5.091 \mathrm{mM}$. The data were means \pm standard deviations of 3 replicates. Means with the same letter after $48 \mathrm{~h}$ were not significantly different. 

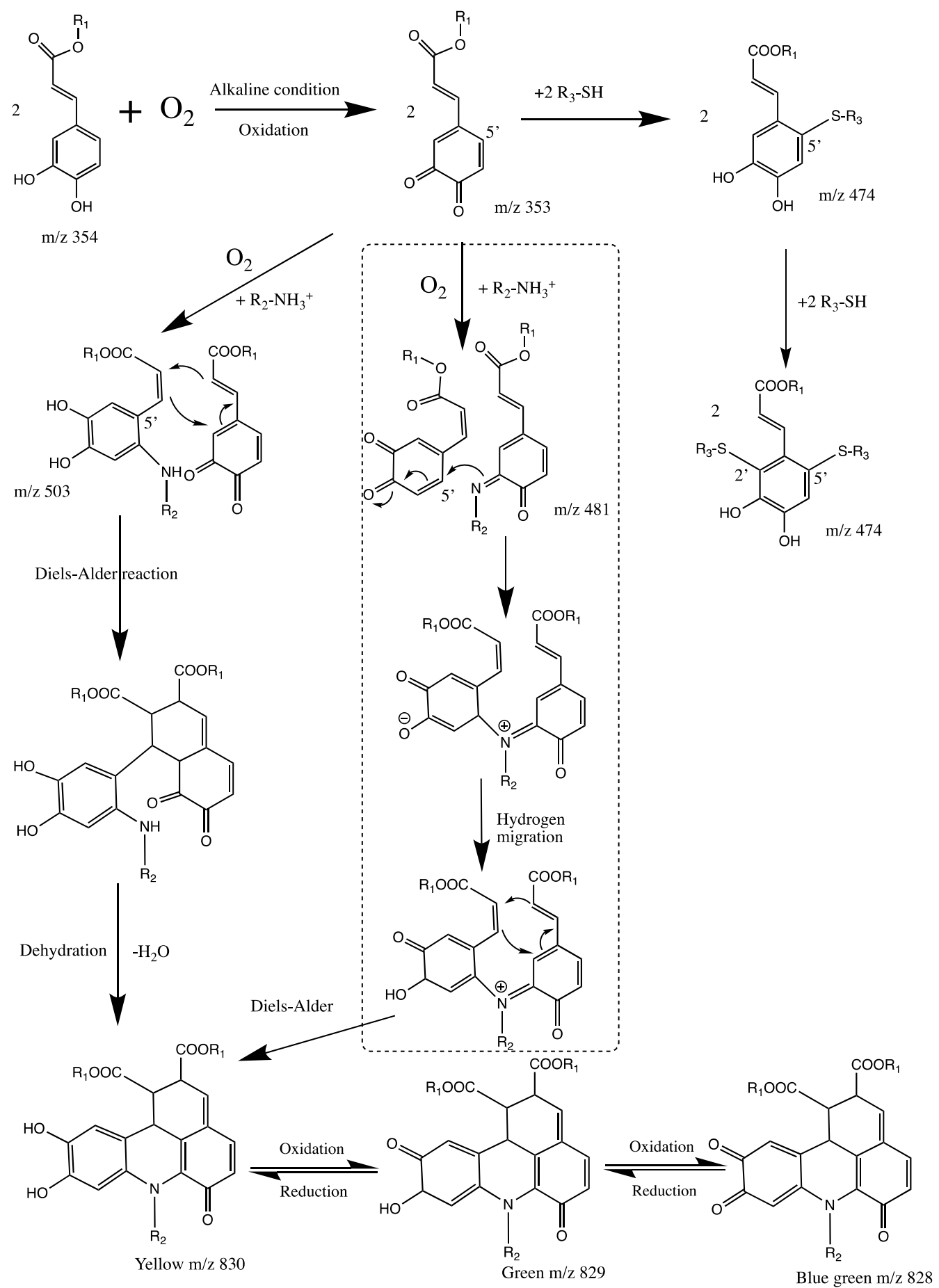

Figure 12 Postulated cysteine inhibition of chlorogenic acid-lysine greening at alkaline $\mathrm{pH}$. $\mathrm{R}_{1}, \mathrm{R}_{2}$, and $\mathrm{R}_{3}$ refers to a quinic acid, lysine and cysteine moiety, respectively. The CGA quinone derivatives may be monomer, dimer or polymers. Dash-line framed route was adapted from Yabuta et al. (2001), which was a potential but less predominant route in our study. 
Given the reducing capacity of thiols, cysteine was able to reduce CGA quinone monomers to CGA hydroquinone dimers (Figure 13).

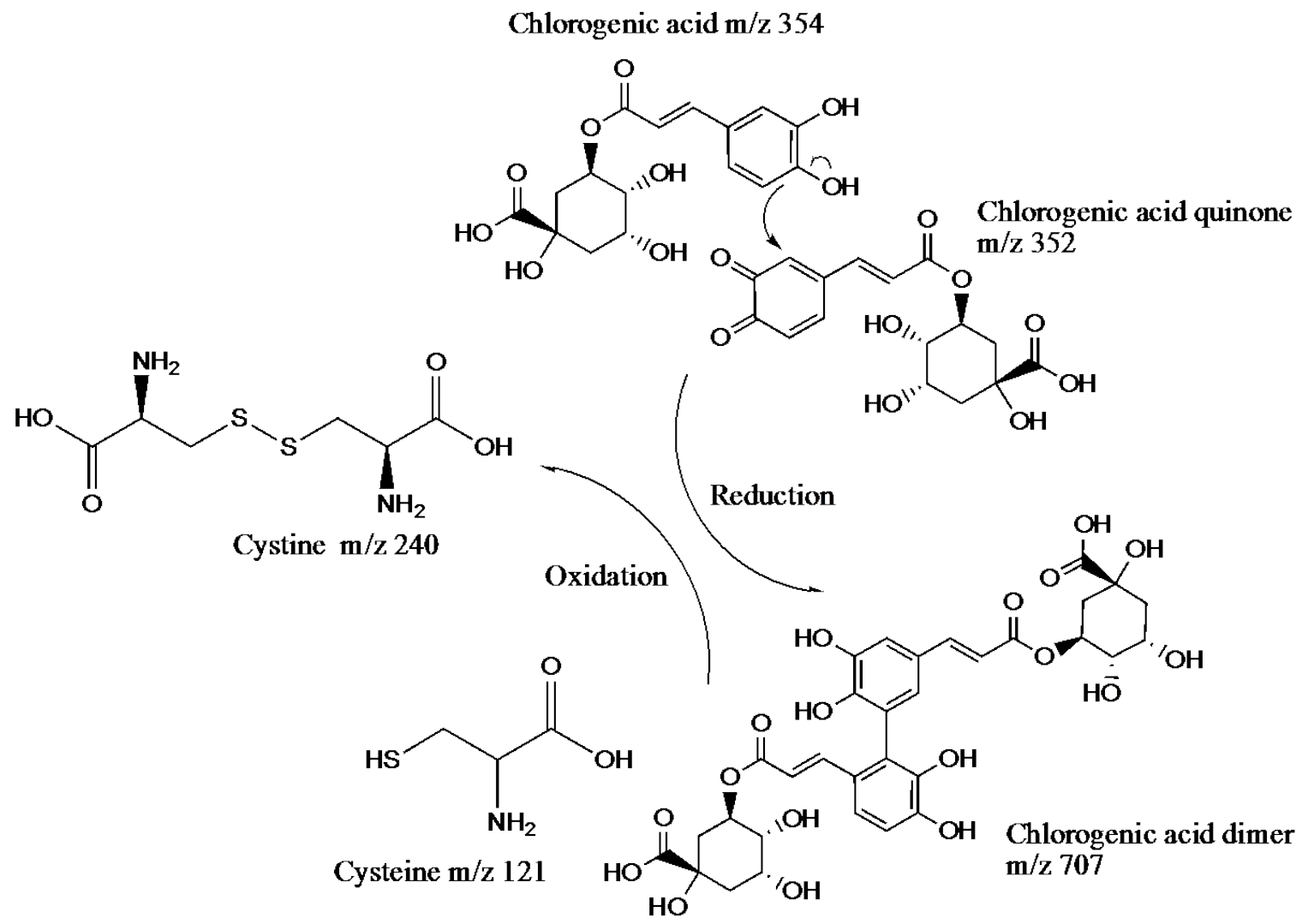

Figure 13 Postulated mechanism of cysteine regenerating chlorogenic acid hydroquinone dimer by redox reaction.

Once regenerated CGA hydroquinone dimers were oxidized to quinones. Dimer CGA quinones can be attacked by amines to form green TBA derivatives or substituted with cysteinyl thiols, consistent with Cao and Xiong (2015) who reported that nucleophilic amine and thiol could conjugate to CGA quinone monomers, and dimers. Overall, changes in cysteine had a significant anti-greening effect (Table 4). 
Table 4 General linear model effect and interaction of $\mathrm{pH}$, cysteine concentration and incubation time on color intensities and antioxidant capacity.

\begin{tabular}{|c|c|c|c|c|c|}
\hline $\begin{array}{l}\text { Independent } \\
\text { variables }\end{array}$ & & $\begin{array}{l}\text { Greening } \\
\left(\text { A680 nm) }^{2}\right.\end{array}$ & $\begin{array}{l}\text { Browning } \\
(\text { A } 410 \mathrm{~nm})\end{array}$ & $\begin{array}{l}\text { Trolox } \\
\text { equivalent } \\
\text { antioxidant } \\
\text { capacity }\end{array}$ & $\begin{array}{l}\text { Folin-Ciocalteu } \\
\text { reagent reducing } \\
\text { capacity }\end{array}$ \\
\hline \multirow[t]{3}{*}{ Cysteine } & $\mathrm{df}^{\mathrm{a}}$ & 5 & 5 & 5 & 5 \\
\hline & $\mathrm{ms}$ & 1.780 & 0.361 & 0.858 & 0.015 \\
\hline & $\mathrm{P}$ & $<0.0001$ & $<0.0001$ & $<0.0001$ & $<0.0001$ \\
\hline \multirow[t]{3}{*}{$\mathrm{pH}$} & $\mathrm{df}$ & 1 & 1 & 1 & 1 \\
\hline & $\mathrm{ms}$ & 1.027 & 87.617 & 2.244 & 0.063 \\
\hline & $\mathrm{P}$ & $<0.0001$ & $<0.0001$ & $<0.0001$ & $<0.0001$ \\
\hline \multirow[t]{3}{*}{ Time } & df & 9 & 9 & 2 & 2 \\
\hline & $\mathrm{ms}$ & 0.903 & 1.915 & 0.139 & 0.028 \\
\hline & $\mathrm{P}$ & $<0.0001$ & $<0.0001$ & $<0.0001$ & $<0.0001$ \\
\hline \multirow{3}{*}{$\begin{array}{l}\text { Interaction } \\
\text { (pH*cysteine) }\end{array}$} & $\mathrm{df}$ & 5 & 5 & 5 & 5 \\
\hline & $\mathrm{ms}$ & 0.062 & 0.041 & 1.536 & 0.035 \\
\hline & $\mathrm{P}$ & $<0.0001$ & $<0.0001$ & $<0.0001$ & $<0.0001$ \\
\hline \multirow{3}{*}{$\begin{array}{l}\text { Interaction } \\
\text { (cysteine*time) }\end{array}$} & df & 45 & 45 & 10 & 10 \\
\hline & $\mathrm{ms}$ & 0.060 & 0.099 & 0.295 & 0.001 \\
\hline & $\mathrm{P}$ & $<0.0001$ & $<0.0001$ & $<0.0001$ & $<0.0001$ \\
\hline \multirow{3}{*}{$\begin{array}{l}\text { Interaction } \\
\text { (pH*time) }\end{array}$} & $\mathrm{df}$ & 9 & 9 & 2 & 2 \\
\hline & $\mathrm{ms}$ & 0.084 & 1.861 & 0.331 & 0.003 \\
\hline & $\mathrm{P}$ & $<0.0001$ & $<0.0001$ & $<0.0001$ & $<0.0001$ \\
\hline \multirow{3}{*}{$\begin{array}{l}\text { Interaction } \\
\text { (pH*Cys*time) }\end{array}$} & $\mathrm{df}$ & 45 & 45 & 10 & 10 \\
\hline & $\mathrm{ms}$ & 0.007 & 0.044 & 0.664 & 0.002 \\
\hline & $\mathrm{P}$ & $<0.0001$ & $<0.0001$ & $<0.0001$ & $<0.0001$ \\
\hline
\end{tabular}

adf refers to degrees of freedom; ms refers to mean square; P refers to $\mathrm{p}$ value.

\subsection{Effect of $\mathrm{pH}$ on brown and green color intensities}

When cysteine concentration was increased from 0 to $1.486 \mathrm{mM}$, greening at $\mathrm{pH}$

9 was strongly negatively correlated with cysteine concentration ( $r=-0.79817$ to -

0.97579, $\mathrm{p}<0.0001$ ) throughout incubation (Figure 9). Moderate and weak correlations between cysteine concentration and greening were observed at $\mathrm{pH} 8$ and $7.75\left(\mathrm{r}_{\mathrm{pH} 8}=\right.$ -

0.70406, $\left.\mathrm{p}=0.0034 ; \mathrm{r}_{\mathrm{pH7} .75}=-0.50525, \mathrm{p}=0.0547\right)$. Stronger correlations between 
cysteine concentration and greening were observed at $\mathrm{pH}$ 9, since $\mathrm{pH} 9$ was above both CGA phenolic pKa 8.42 and cysteine thiol pKa 8.3. This greater anti-greening effect by cysteine at higher $\mathrm{pH}$ was consistent with observations in buffered $\mathrm{pH} 8$ and 9 CGA: Lys: Cys solutions when cysteine was increased to $5.09 \mathrm{mM}$ (Figure 11 C\&D).

The phenolic pKa of CGA of 8.42 is lower than that in $\mathrm{pH} 9$ solutions resulting in higher browning in buffered $\mathrm{pH} 9$ than $\mathrm{pH} 8$ solutions (Figure 11A\&B). At alkaline $\mathrm{pH}$, only CGA phenolates were oxidized to brown CGA o-quinone monomers, so lower $\mathrm{pH}$ has a protective effect on free CGA (Figure 16C). As phenolic ions gave rise to CGA oquinone formation (Cilliers \& Singleton, 1989), initial browning in pH 9 buffered solutions was twice as high as in $\mathrm{pH} 8.0$ buffered solutions ( $<<0.0001$, Figure $11 \mathrm{~A} \& \mathrm{~B}$ ).

Highly electrophilic CGA o-quinones formed by non-enzymatic oxidation could be stabilized by both thiol and amine nucleophilic addition (Penalver et al., 2002). When amines nucleophilically attacked CGA quinones, formation of green trihydroxy benzacridine led to hyperchromic shift at $680 \mathrm{~nm}$ after $48 \mathrm{~h}$ in buffered CGA: Lys: Cys solutions at all tested pHs and cysteine concentrations (Figure $11 \mathrm{C} \& \mathrm{D}$ ). Greening in $\mathrm{pH}$ 9.0 CGA: lysine: cysteine solutions was significantly higher than that in $\mathrm{pH} 8.0$ buffered solutions $(\mathrm{p}<0.0001)$ at each tested cysteine concentration. Higher greening in $\mathrm{pH} 9$ buffered solutions was attributed to deprotonation of both CGA (pKa of phenol: 8.42) and lysine (pKa of $\alpha$-amine: 8.95). At pH 9, besides facilitating CGA o-quinones formation, deprotonation of $\alpha$-lysyl amine group (pKa 8.90) made $\varepsilon$-lysyl amine group (pKa 10.28), the primary site to covalently bind with CGA $o$-quinones to form aminequinone adducts, followed by Diels-Alder cyclization and dehydration to yellow pigmented TBA derivatives (Figure 12), which became green once oxidized (Yabuta et 
al., 2001). In pH 8.0 buffered solutions, lower greening after $48 \mathrm{~h}$ incubation occurred because less $o$-quinones were available at less alkaline $\mathrm{pH}$ to form green TBA derivatives and CGA o-quinones have a lower reactivity in less alkaline conditions (Bongartz et al., 2016). At the same cysteine concentration, pH 9 CGA: Lys: Cys solutions had significantly higher greening because higher $\mathrm{pH}$ promoted oxidative reactions including formation of CGA-quinones and TBA. More thiyl radicals would form at higher $\mathrm{pH}$, facilitating oxidation to green TBA derivatives. In addition, more thiyl radicals gave rise to cysteinyl-CGA conjugates. Thus, greater inhibition of greening was observed at $\mathrm{pH} 9$ than 8 , as $5.09 \mathrm{mM}$ cysteine inhibited $94.0 \%$ greening at $\mathrm{pH} 9$ compared to $86.8 \%$ greening inhibition at $\mathrm{pH} 8$.

\subsection{Formation of reaction products over incubation}

At alkaline pH, electrophilic CGA-quinones (353 Da, Figure 12) were subject to nucleophilic attack by amines or thiols in buffered CGA: Lys: Cys solutions. When the highly reactive CGA-quinones (353 Da) were stabilized by amine addition, aminehydroquinone adducts (499 Da) were formed, which were subsequently cyclized with another CGA-quinone by Diels-Alder reaction and oxidative elimination to form a trihydroxy benzacridine (TBA) structure (830 Da). The hydroxy groups oxidized into ketones at alkaline $\mathrm{pH}(829,828 \mathrm{Da}$, Figure 12), and thus green pigmentation was observed in pH 8 and 9 CGA: Lys: Cys solutions except with 5.09 mM cysteine. Formation of TBA derivatives led to a hyperchromic shift at $680 \mathrm{~nm}$ in $\mathrm{pH} 8$ and 9 buffered CGA: Lys: Cys solutions (Figure 11 C\&D), implying a simultaneous consumption of brown CGA quinones (correlations for browning and cysteine concentration: $\mathrm{r}_{\mathrm{pH} 8}=-0.5707$ to $0.9149 ; \mathrm{r}_{\mathrm{pH9}}=-0.7130$ to 0.9551$)$. 
Table 5 Relative abundance of eluted compounds at 1.60 and 1.75 minutes by HPLC peak area at $320 \mathrm{~nm}$.

\begin{tabular}{|c|c|c|c|c|c|c|}
\hline & pH 8 & & & pH 9 & & \\
\hline $\begin{array}{l}\text { Cysteine } \\
(\mathbf{m M})\end{array}$ & $8 \mathrm{~h}$ & $24 \mathrm{~h}$ & $48 \mathrm{~h}$ & $8 \mathrm{~h}$ & $24 \mathrm{~h}$ & $48 \mathrm{~h}$ \\
\hline \multicolumn{7}{|c|}{ Compound eluted at $1.60 \mathrm{~min}^{\mathrm{a}}$} \\
\hline 0 & 740.57 & 1457.23 & 1870.00 & 3268.67 & 3457.27 & 3891.87 \\
\hline 1.02 & 710.26 & 1345.35 & 1736.49 & 2979.39 & 3414.17 & 3385.75 \\
\hline 1.27 & 683.81 & 1405.27 & 1701.61 & 2834.30 & 3281.49 & 3371.49 \\
\hline 2.55 & 832.99 & 1457.65 & 1411.35 & 2287.25 & 3049.73 & 3123.02 \\
\hline 3.82 & 953.79 & 1414.71 & 1434.47 & 2036.34 & 2682.04 & 2785.24 \\
\hline 5.09 & 896.76 & 1214.52 & 1539.28 & 1885.01 & 2519.79 & 2657.22 \\
\hline \multicolumn{7}{|c|}{ Compound eluted at $1.75 \mathrm{~min}^{\mathrm{b}}$} \\
\hline 0 & $1.03 \mathrm{E}+04$ & 7382.13 & 6300.74 & 5348.54 & 2365.87 & 2284.20 \\
\hline 1.02 & $1.14 \mathrm{E}+04$ & 7450.91 & 6426.08 & 5552.52 & 3601.03 & 2574.61 \\
\hline 1.27 & $1.05 \mathrm{E}+04$ & 8244.17 & 7048.81 & 4135.43 & 2738.76 & 3101.64 \\
\hline 2.55 & $1.09 \mathrm{E}+04$ & 8299.54 & 7821.39 & 6666.02 & 4958.46 & 3996.77 \\
\hline 3.82 & $1.10 \mathrm{E}+04$ & $1.01 \mathrm{E}+04$ & 8358.80 & 7487.74 & 5222.59 & 4976.34 \\
\hline 5.09 & $1.13 E+04$ & 8313.35 & 7971.60 & 8352.71 & 6646.72 & 5647.92 \\
\hline
\end{tabular}

${ }^{\mathrm{a} C o m p o u n d}$ eluted at 1.60 min was determined to be green trihydroxy benzacridine derivatives.

${ }^{\mathrm{b}}$ Compound eluted at 1.75 min was suspected to be amine/thiol-CGA conjugates. 
A. pH 8 buffered CGA: Lys: Cys solutions absorbance at $320 \mathrm{~nm}$

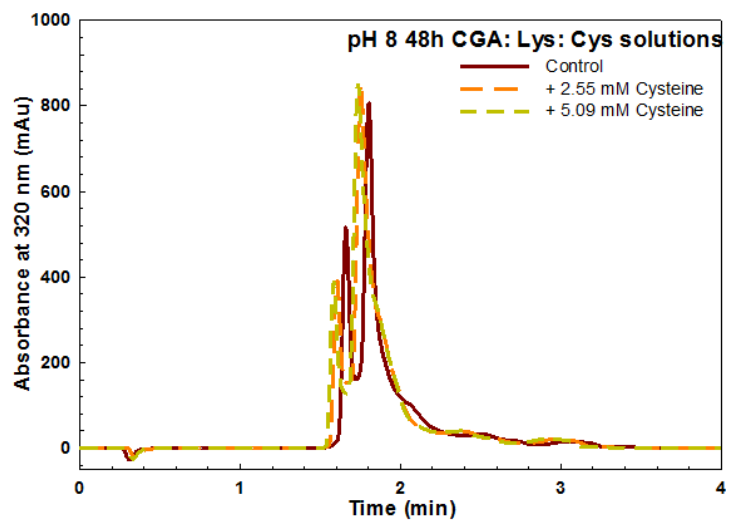

C. pH 8 buffered CGA: Lys: Cys solutions absorbance at $450 \mathrm{~nm}$

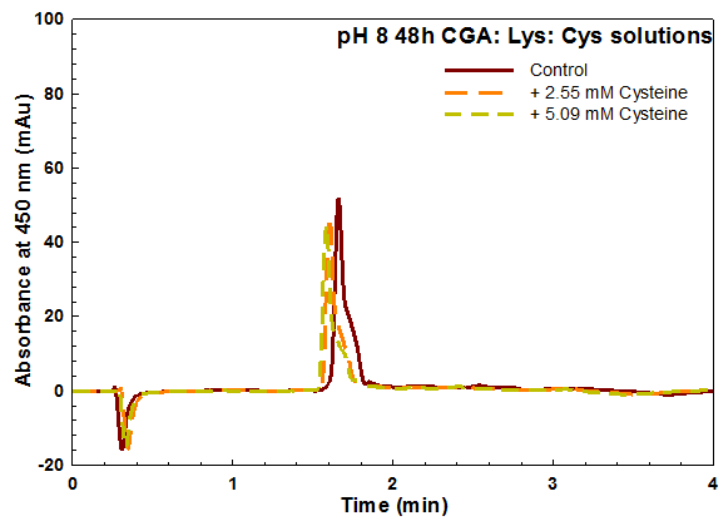

E. pH 8 buffered CGA: Lys: Cys solutions absorbance at $680 \mathrm{~nm}$

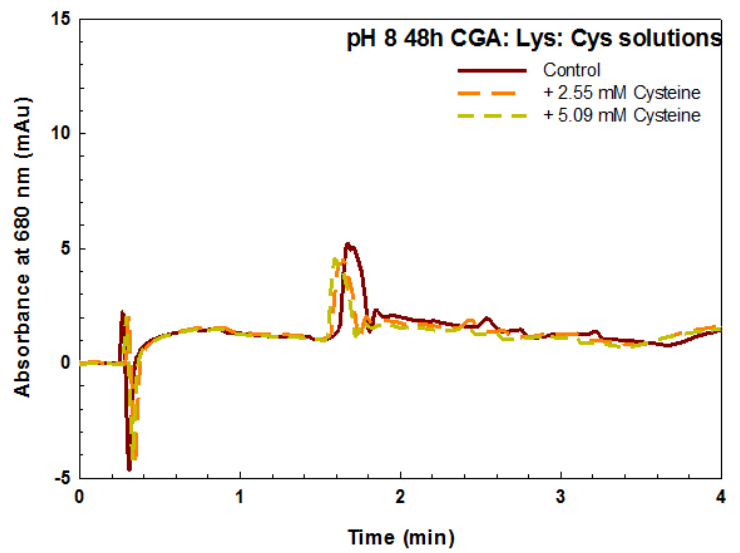

B. pH 9 buffered CGA: Lys: Cys solutions absorbance at $320 \mathrm{~nm}$

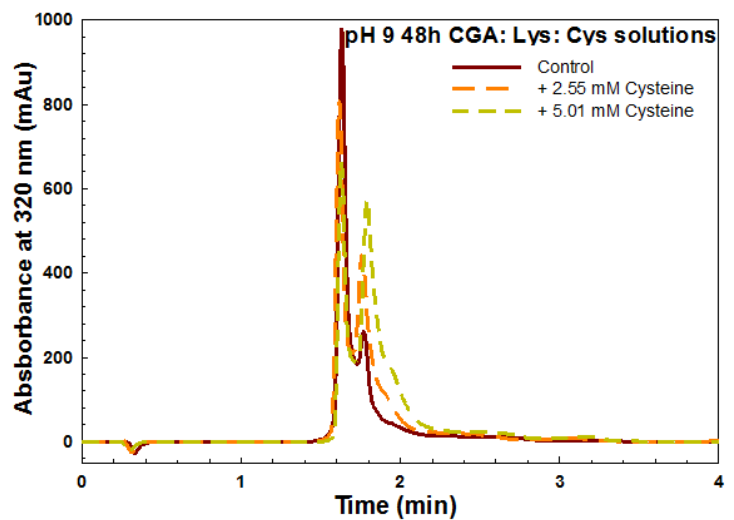

D. pH 9 buffered CGA: Lys: Cys solutions absorbance at $450 \mathrm{~nm}$

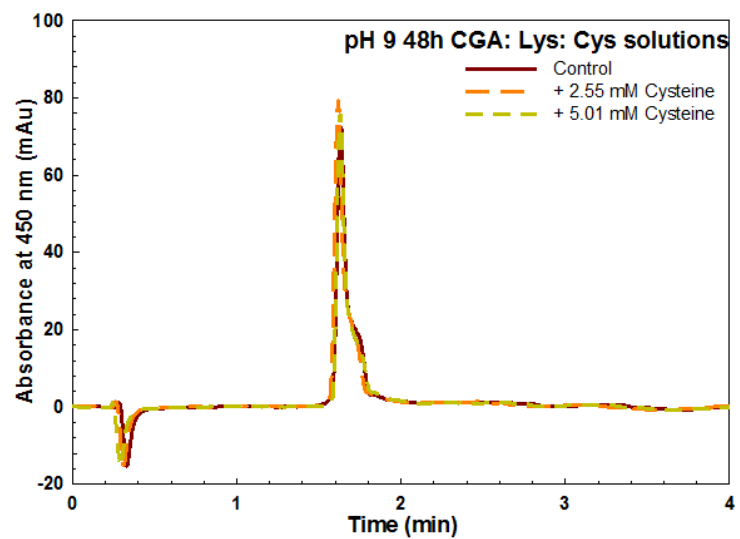

F. pH 9 buffered CGA: Lys: Cys solutions absorbance at $680 \mathrm{~nm}$

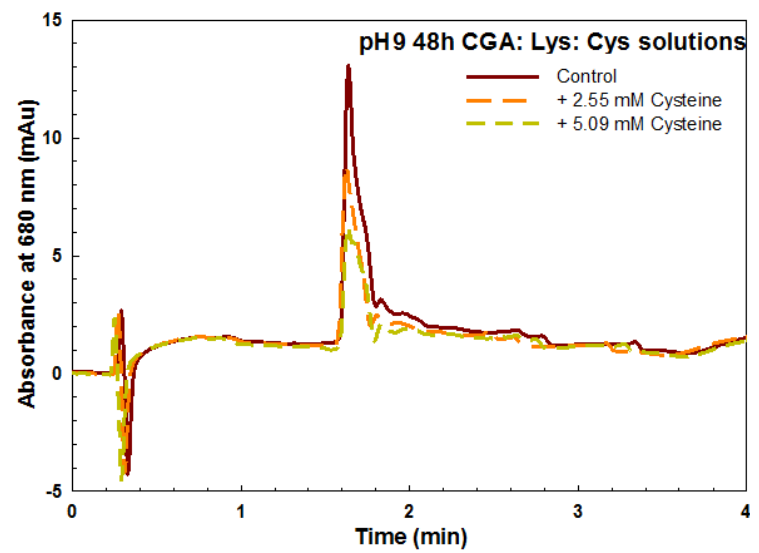

Figure 14 HPLC chromatograms at $320 \mathrm{~nm}$ (A, B), $450 \mathrm{~nm}$ (C, D), and $680 \mathrm{~nm}$ (E, F) of pH 8 and 9 buffered CGA: Lys: Cys solutions ( Control, $-+2.55 \mathrm{mM}$ Cysteine,$---+5.01 \mathrm{mM}$ Cysteine $)$ after $48 \mathrm{~h}$ incubation at ambient temperature. 
Formation of green TBA derivatives was inhibited at $5.09 \mathrm{mM}$ cysteine concentration and lowered pH (Figure $11 \mathrm{C} \& D$ ), consistent with HPLC data shown in Figure 14. Two absorbance peaks were observed at $320 \mathrm{~nm}$ (Figure $14 \mathrm{~A} \& \mathrm{~B}$ ): one at 1.60 minutes, the other eluted at 1.75 minutes. The three-way interactions between cysteine concentration $\mathrm{X} \mathrm{pH} \mathrm{X}$ storage time were significant for compounds eluting at the two times, 1.60 and 1.75 minutes (Table 4, Table 5). The compounds eluting at 1.60 minutes were detected at all $3 \lambda \mathrm{s}: 320,450$ and $680 \mathrm{~nm}$, and were thus identified as green TBA derivatives. After 48h, the relative abundance of TBA derivatives given by HPLC absorbance peak area decreased from 1870.00 to 1539.28 and from 3891.87 to 2657.22, as cysteine increased at pH 8 and 9, respectively (Table 5), which was consistent with hypochromic shift at $680 \mathrm{~nm}$ at $\mathrm{pH} 8$ and 9 (Figure $11 \mathrm{C} \& \mathrm{D}$ ).

Signals of compounds that eluted at 1.75 minutes were only detected at $320 \mathrm{~nm}$ but not $450 \mathrm{~nm}$ and $680 \mathrm{~nm}$, indicating a hydroxycinnamic acid (HCA) based phenolic structure (C3-C6 phenolics). Since CGA and caffeic acid standards eluted at 2.40 and 2.71 minutes respectively, the compounds that eluted at 1.75 minutes were possibly CGA conjugates, whose relative abundance increased from 6300.74 to 7971.60 and from 2284.2 to 5647.92, as cysteine increased at $\mathrm{pH} 8$ and 9, respectively. The 1.75 min eluted compounds were tentatively identified as co-eluted lysyl amine-CGA and cysteinyl-CGA conjugates because the peak height decreased while the peak bottom became wider (peak tailing) as cysteine concentration increased and the relative abundance in controls were not zero at both $\mathrm{pH}$. In addition, the relative abundance of this compound(s) decreased throughout incubation (Table 5). It was thus concluded that CGA quinones react with lysyl amines to form amine-CGA conjugates before cyclization with another CGA 
quinone to form green TBA derivatives (Figure 12).

Lysyl amine-CGA conjugates and green TBA derivatives were identified in all CGA: Lys: Cys solutions at pH 8 and 9. Compound 2 (Table 6, Figure 15B) was identified as a lysine-CGA conjugate with a sodium ion, as all CGA: Lys: Cys solutions were prepared in sodium phosphate buffer. The predominant fragment $[\mathrm{M}+\mathrm{H}]+$ of compound 1 was at m/z 147, consistent with a lysine moiety in the structure, which can be further fragmented to m/z 130 as $\mathrm{C}_{6} \mathrm{H}_{12} \mathrm{NO}_{2}$ (2-Aminohexanoate isomer), and m/z 84 as $\mathrm{C}_{4} \mathrm{H}_{6} \mathrm{NO}$ (1-Oxo-2-buten-2-yl-amino). Ion m/z 311 was 164 Da higher than fragment at $\mathrm{m} / \mathrm{z} 147$, indicating a $\mathrm{C}_{9} \mathrm{H}_{8} \mathrm{O}_{3}$ ion (caffeic aldehyde isomer) was attached.

The precursor ion m/z 527 was 23 Da higher than predicted lysine-CGA conjugate at $\mathrm{m} / \mathrm{z}$ 504, suggesting a sodium ion was attached to lysine-CGA conjugates (Figure 15B). Higher signal intensity (89433 at pH 8; 79336 at pH 9) of compound 2 in pH 8 than $\mathrm{pH} 9$ solutions at the same cysteine concentrations, indicated that lysyl amineCGA conjugate was a precursor of green TBA in alkaline CGA: Lys: Cys solutions, more of which was consumed in pH 9 than 8 solutions. Lysine-CGA iminoquinone (m/z 481, Figure 12) was postulated as an intermediate of green TBA formation (Yabuta et al., 2001), which was not a predominant reaction product identified in this study. Given the high abundance of lysyl amine-CGA conjugates (Figure 15B), lysine reacting with CGA quinones preferentially to form lysyl amine-CGA conjugates, which was 10 times more than cysteinyl-CGA conjugates, followed by a dehydro-Diels-Alder reaction was the primary route to form green TBA (Figure 12).

Compound 5 (Table 6, Figure 15E) was identified as trihydroxy benzacridine (TBA, m/z 830) minus a carboxy group (44 Da) on a quinic acid moiety (174 Da). Ion 
$\mathrm{m} / \mathrm{z} 263$ was a trihydroxy benzacridine core structure, $\mathrm{C}_{16} \mathrm{H}_{8} \mathrm{NO}_{3}$ (possibly a 6-Oxo-2,6dihydronaphtho-indole-1-carboxylate), consistent with TBA fragments reported by Bongartz et al. (2016) and Schilling et al. (2008). Ion m/z 525 was a trihydroxy benzacridine core structure, $\mathrm{C}_{16} \mathrm{H}_{8} \mathrm{NO}_{3}$, attached with a quinic acid (191 Da), a carboxy group (44 Da) and a carbonyl group (28 Da), when a quinic acid moiety (174 Da) and a lysine moiety (129 Da) were fragmented from a parent TBA molecule (m/z 830). Higher signal intensity of compound 5 in $\mathrm{pH} 9$ than $\mathrm{pH} 8$ solutions was consistent with TBA quantification by HPLC (Table 5) where higher concentrations of green TBA derivatives were formed at higher $\mathrm{pH}$.

Cysteine reacted with CGA-quinones in alkaline CGA: Lys: Cys solutions primarily by redox phenol regeneration with formation of cystine (240 Da) and CGA dimers (707 Da, Figure 13). Compound 6 and 7 were in high abundance and were identified as cystine-cystine crosslinks (m/z 481, Figure 15F) and CGA dimers (m/z 708) respectively. Compound 7 (m/z 731) was 23 Da higher than a CGA dimer (m/z 708, Figure 15G), identified as a CGA dimer with a sodium ion, whose abundance was $60 \%$ higher in $\mathrm{pH} 8$ than $\mathrm{pH} 9$ solutions. Thus, cysteine was more prone to regenerate CGA diphenols at pH 8 when $\mathrm{pH}$ was below its thiol pKa 8.3 . 
Table 6 Identification of reaction products in $\mathrm{pH} 8$ and 9 buffered chlorogenic acid /CGA: Lys: Cys solutions after $48 \mathrm{~h}$ incubation.

\begin{tabular}{|c|c|c|c|c|}
\hline Compound & $\begin{array}{l}\text { Retention } \\
\text { time } \\
\text { (min) }\end{array}$ & {$[\mathbf{M}+\mathbf{H}]^{+}$} & $\begin{array}{l}\text { MS fragments } \\
\text { (\%relative } \\
\text { abundance) }\end{array}$ & Tentative identification \\
\hline 1 & 2.3 & 430.91 & $\begin{array}{l}81 \text { (18.1), } 91 \text { (56.6), } \\
159 \text { (29.0), } 217(55.4), \\
219 \text { (17.1), } 227(100), \\
243 \text { (14.2), } 363 \text { (13.8), } \\
421 \text { (13.2), } 431(12.6)\end{array}$ & $\begin{array}{l}\text { Cysteinyl-CGA conjugate (475) - } \\
\text { Carboxyl group (44) }\end{array}$ \\
\hline 2 & 4.1 & 527.25 & $\begin{array}{l}84(12.1), 130 \text { (55.6), } \\
147(100), 148(7.1), \\
169 \text { (26.6), } 191(4.5), \\
289 \text { (5.3), } 311(5.2), \\
359(4.1), 527(4.0)\end{array}$ & $\begin{array}{l}\text { Lysine-CGA conjugates }(504)+ \\
{\left[\mathrm{Na}^{+}\right]}\end{array}$ \\
\hline 3 & 4.4 & 946.84 & $\begin{array}{l}91 \text { (33.2), } 159 \text { (16), } \\
227 \text { (100), } 363 \text { (19.9), } \\
431 \text { (28.2), } 499 \text { (8.9), } \\
567 \text { (10.6), } 635 \text { (12.7), } \\
703 \text { (12.3), } 947(6.9)\end{array}$ & $\begin{array}{l}\text { Cysteinyl-CGA-Cysteinyl-CGA } \\
\text { crosslink (947) }\end{array}$ \\
\hline 4 & 4.5 & 662.82 & $\begin{array}{l}91 \text { (40.8), } 159 \text { (33), } \\
227 \text { (100), } 255 \text { (14.8), } \\
323 \text { (26.3), } 391 \text { (18.9), } \\
459 \text { (23.2), } 527(24.4), \\
595 \text { (24.4), } 663 \text { (18.6) }\end{array}$ & $\begin{array}{l}\text { Di-cysteinyl-CGA conjugate (595) } \\
+ \text { Carboxyl group (44) }+\left[\mathrm{Na}^{+}\right]\end{array}$ \\
\hline 5 & 4.6 & 786.71 & $\begin{array}{l}143 \text { (100), } 165 \text { (86.1), } \\
263 \text { (70.5), } 285 \text { (56.0), } \\
383 \text { (44.5), } 405 \text { (65.8), } \\
525 \text { (49.0), } 645 \text { (48.7), } \\
667 \text { (40.2), } 787 \text { (40.5) }\end{array}$ & $\begin{array}{l}\text { Trihydroxy benzacridine (830) - } \\
\text { Carboxyl group (44) }\end{array}$ \\
\hline 6 & 5 & 480.84 & $\begin{array}{l}86 \text { (72.5), } 99 \text { (100), } \\
100 \text { (44.1), } 125 \text { (58.6), } \\
146 \text { (42.9), } 241(58.0), \\
263 \text { (56.6), } 361 \text { (45.3), } \\
459 \text { (42.7), } 481 \text { (51.6) }\end{array}$ & Cystine-cystine crosslink (480) \\
\hline 7 & 8.7 & 731.18 & $\begin{array}{l}163 \text { (31.4), } 164(4.5), \\
225 \text { (6.6), } 355 \text { (24.9), } \\
377(100), 378(13.0), \\
399 \text { (27.5), } 435(5.7), \\
457 \text { (7.0), } 731(9.6)\end{array}$ & $\begin{array}{l}\text { Chlorogenic acid dimer (708) + } \\
{\left[\mathrm{Na}^{+}\right]}\end{array}$ \\
\hline
\end{tabular}

At $\mathrm{pH}$ 9, more cysteine reacted with CGA o-quinones by thiol-conjugation: thiyl radical nucleophilically added to yellow electrophilic o-quinones to form colorless thiolether conjugates, cysteinyl-CGA conjugates, which were subject to secondary thiol substitution to form di-cysteinyl CGA conjugates (Cilliers \& Singleton, 1990; Schilling et 
al., 2008). Both mono-cysteinyl-CGA (compound 1), and di-cysteinyl-CGA (compound 4) conjugates were identified in alkaline CGA: Lys: Cys solutions. Compound 1 (Table 6, Figure 15A) was recognized as mono-cysteinyl-CGA conjugate (m/z 475) lost a carboxyl group (44 Da). The predominant fragment $[\mathrm{M}+\mathrm{H}]^{+}$of compound 1 was at $\mathrm{m} / \mathrm{z} 227$, which was a thiol-methyl caffeate conjugate, $\mathrm{C}_{10} \mathrm{H}_{10} \mathrm{O}_{4} \mathrm{~S}$. Ion m/z 431 was 164 Da higher than fragment at $\mathrm{m} / \mathrm{z} 147$, indicating a $\mathrm{C}_{9} \mathrm{H}_{8} \mathrm{O}_{3}$ ion was attached. As $\mathrm{pH}$ decreased from 9 to 8 , $50 \%$ decreased signal intensity of compound 1 was observed when cysteine concentration was kept constant. Compound 3 (Table 6), which fragmented in a similar pattern as compound 1 did, was identified as a 2 mono-cysteinyl-CGA conjugate (m/z 475) crosslinked together (m/z 947). Compound 4 (Table 6, Figure 15D) was identified as dicysteinyl-CGA conjugates (m/z 595) plus a carboxy group with a sodium ion. The predominant fragment $[\mathrm{M}+\mathrm{H}]^{+}$was the same as that of compound 1 and 3 , a thiol-methyl caffeate conjugate, $\mathrm{C}_{10} \mathrm{H}_{10} \mathrm{O}_{4} \mathrm{~S}$, at m/z 227. Parent ion m/z 663 was 68 Da higher than dicysteinyl-CGA conjugates at $\mathrm{m} / \mathrm{z}$ 595, which could be a protonated carboxy group (45 Da) and a sodium ion (23 Da). More di-cysteinyl CGA conjugates were formed in pH 9 than pH 8 CGA: Lys: Cys solutions. pH 8.0 solutions were below cysteinyl thiol pKa ( 8.3), and the ratio of thiyl radical (-S-) to sulfhydryl (-SH) was higher in $\mathrm{pH} 9.0$ than 8.0 buffered solutions, so cysteinyl thiols were more prone to form colorless cysteinylCGA conjugates at $\mathrm{pH} 9$ while more reductively regenerated CGA dimers were found in CGA: Lys: Cys solutions. However, brown solutions were observed in CGA: Lys: 5.09 mM Cys solutions (Figure 10 \& Figure 11C\&D). As imine formation was retarded with presence of cysteine (Zeng et al., 2017; Zhang et al., 2018), hypochromic shift at $410 \mathrm{~nm}$ in non-green solution might be induced by oxidation cysteinyl-CGA conjugates. 
Overall, at the same alkaline $\mathrm{pH}$, the abundance of mono-cysteinyl CGA conjugates increased while the formation of lysyl amine-CGA conjugates was not influenced by cysteine concentration from 0 to $5.09 \mathrm{mM}$. When cysteine was kept constant at $5.09 \mathrm{mM}$, no visible greening was observed at either $\mathrm{pH} 8$ or $\mathrm{pH}$ 9, and simultaneously the amount of CGA dimers in pH 8 was 60\% higher than in pH 9 solutions, indicating cysteine was primarily reducing brown CGA o-quinones to CGA hydroquinone dimers when $\mathrm{pH}$ was below cysteinyl thiol pKa 8.3. 
A. Compound 1: Cysteinyl-CGA conjugate $(\mathrm{m} / \mathrm{z} 475)$ minus a carboxyl group (44

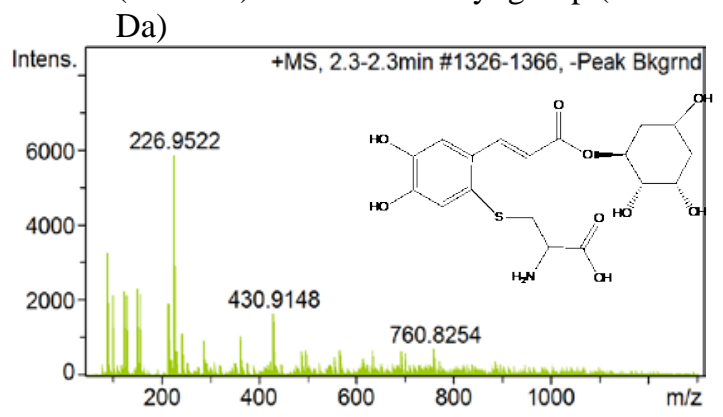

C. Compound 3: Mono-cysteinyl CGA crosslink (m/z 947)

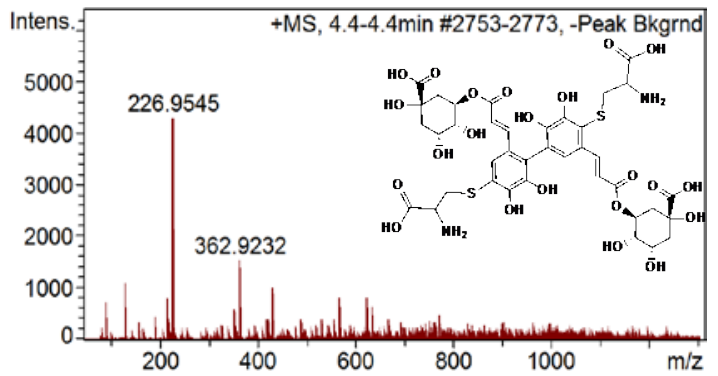

E. Trihydroxy benzacridine ( $\mathrm{m} / \mathrm{z} 830)$ minus a carboxyl group (44 Da)

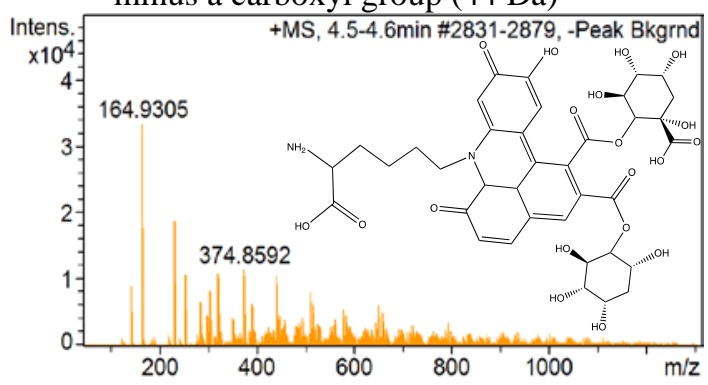

B. Compound 2: Lysyl amine-CGA conjugate $(\mathrm{m} / \mathrm{z} 504)+\left[\mathrm{Na}^{+}\right]$

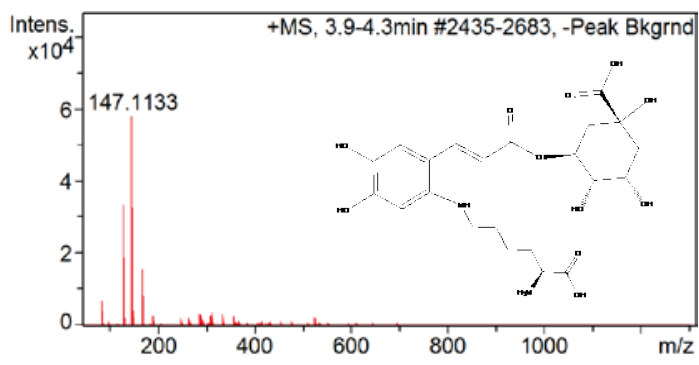

D. Compound 4: Di-cysteinyl CGA conjugate $(\mathrm{m} / \mathrm{z} 595)+$ carboxyl group $(44 \mathrm{Da})+$ $\left[\mathrm{Na}^{+}\right]$

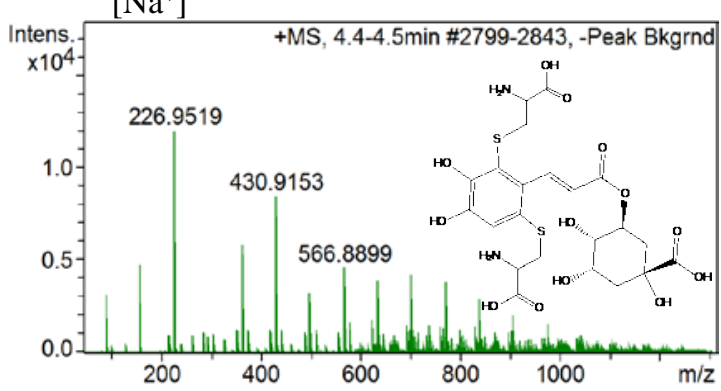

F. Cystine-cystine crosslink (m/z 481)

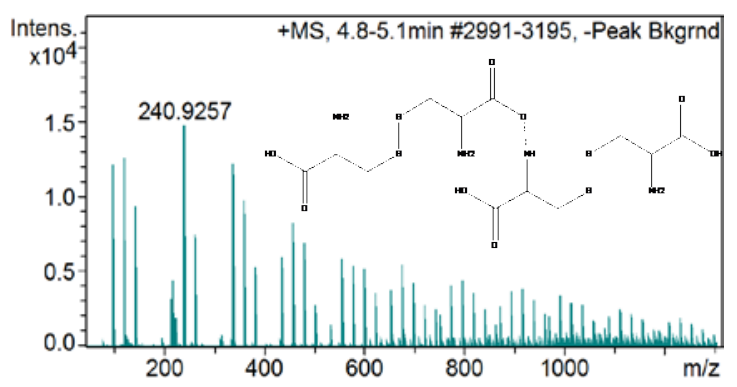

G. Chlorogenic acid dimer $(\mathrm{m} / \mathrm{z} 708)+\left[\mathrm{Na}^{+}\right]$

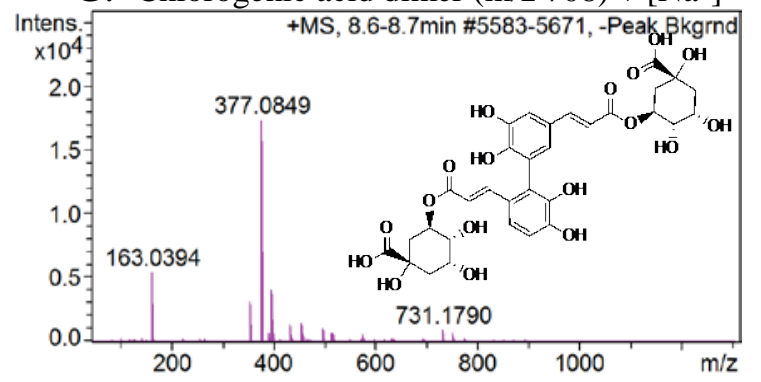

Figure 15 Mass spectrometric chromatograms and chemical structures of identified compounds in $\mathrm{pH} 8$ and 9 chlorogenic acid: Lys: Cys solutions.

A to $\mathrm{G}$ correspond to compound 1 to 7 in Table 6 . 


\subsection{Cysteine effects on Trolox equivalents antioxidant capacity (TEAC) and Folin- Ciocalteu Reagent reducing Capacity (FCRC) in CGA: Lys: Cys solutions}

Cysteinyl thiol and di-thiol CGA conjugates were expected to exhibit antioxidant capacity, given similarity of caffeic acid and CGA, an ester of caffeic acid. Fujimoto et al. (2013) reported mono-thiol methyl caffeate adduct scavenged ethyl linoleate hydroperoxide radicals nearly twice as much as methyl caffeate did. Miura et al. (2014) found cysteinylcaffeic acids successfully maintained the desirable bright red color of meat by reducing metmyoglobin to oxymyoglobin.

Antioxidant capacity by Trolox equivalent antioxidant (TEAC) assay primarily measures the potential to neutralize free radicals, as TEAC measures the ionization potential equivalent. Antioxidant capacity by TEAC at pH 8 was significantly higher than pH 9 (Figure 16A), because $\varepsilon$-lysyl amine became more reactive at pH 9 than $\mathrm{pH}$ 8. Thus more CGA was consumed to produce green TBA and less free CGA was present in pH 9 solutions (Figure 16C). As cysteine concentration increased, TEAC at both pH 8 and 9 increased as more mono-cysteinyl-CGA conjugates (Figure 15A\&C) were formed. Plateaued TEAC was observed at both tested $\mathrm{pH}$ when cysteine was higher than 1.27 mM, consistent with Fujimoto et al. (2013) who found that additional cysteinyl thiol would not cause further antioxidant capacity enhancement, as excess cysteine might be consumed by secondary thiol substitution (Figure 15D).

Antioxidant capacity by FCRC assay assessing electron transferring ability (Gabriel, Joe, \& Patrick, 2014), showed that increasing FCRC was observed at both tested $\mathrm{pH}$ as cysteine concentration increased, except in $\mathrm{pH} 8$ control. Higher FCRC in pH 8 control might be attributed to the reducing capacity of free CGA (Figure 16B). 
Higher quantity of unreacted CGA in pH 8 control after 48 h (Figure 16C) was because less amino acids were available to form CGA conjugates.

Overall, lowering pH from 9 to 8 resulted in 4.7\%-22\% higher FCRC and a 7.3\%-36\% higher TEAC because cysteine protected more CGA at pH 8 by reducing CGA quinones to CGA hydroquinone dimers, which had 60\% higher signal intensity in pH 8 than pH 9 solutions (Figure 15G). As cysteine concentration increased from 1.02 $\mathrm{mM}$ to $5.09 \mathrm{mM}, 20 \%$ and $39 \%$ higher FCRC were found when $\mathrm{pH}$ remained the same at 8 and 9 respectively. When cysteine concentration was kept constant, reducing capacity was increased when $\mathrm{pH}$ was lowered from 9 to 8, indicating antioxidant capacity could be enhanced by lowering pH. Compared to controls with no added cysteine, FCRC at pH 8 was nearly $60 \%$ higher than that at $\mathrm{pH} 9$. With addition of cysteine, the FCRC increased to a greater extent (21.5\%) at $1.02 \mathrm{mM}$ cysteine while it only increased by $4.7 \%$ at 5.09 $\mathrm{mM}$ cysteine when $\mathrm{pH}$ was lowered from 9 to 8, suggesting the enhanced reducing capacity might be due to formation of cysteinyl-CGA conjugates instead of increased cysteine in the solutions. Although adding cysteine and lowering $\mathrm{pH}$ both enhanced antioxidant capacity ( $<<0.0001$, Table 4), cysteine exhibited a greater effect. The interaction effect of cysteine concentration and $\mathrm{pH}$ enhanced more on reducing than radical scavenger capacity (Table 4). 
A. Trolox equivalent antioxidant capacity (mM) as a function of cysteine concentration

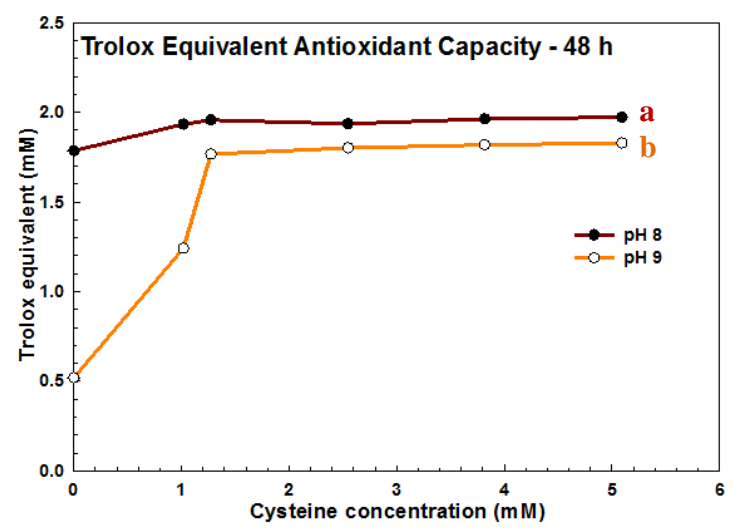

B. Gallic acid equivalent reducing capacity $(\mathrm{mg} / \mathrm{mL})$ as a function of cysteine concentration

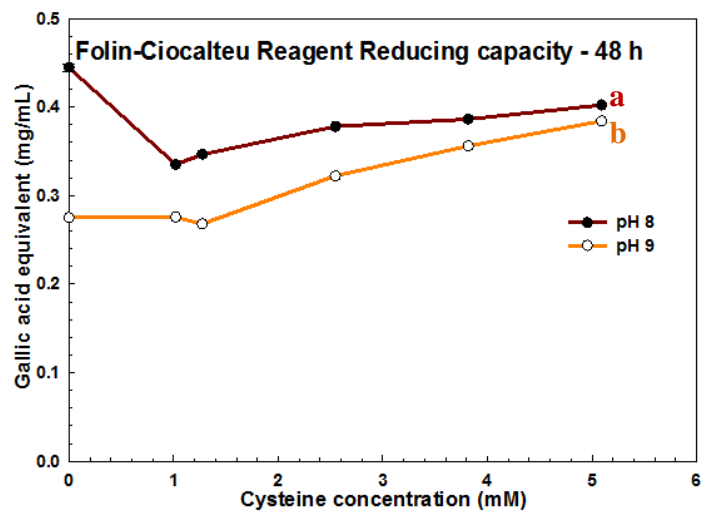

C. Free CGA (mM) as a function of cysteine concentration

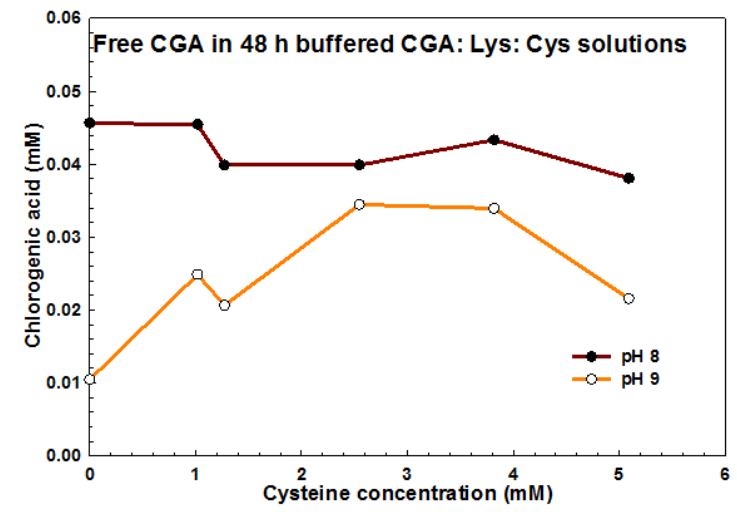

Figure 16 Trolox equivalents antioxidant capacity (A) and gallic acid equivalent reducing capacity (B) as a function of cysteine concentration (0 - $5.091 \mathrm{mM})$ in buffered $\longrightarrow \mathrm{pH} 8$ and $-\mathrm{o}-\mathrm{pH} 9$ CGA: Lys: Cys solutions.

\section{Summary and Conclusions}

Adding cysteine to buffered pH 8 and 9 CGA-lysine mixtures inhibited CGA quinone induced greening in a concentration dependent manner by forming cysteinylCGA conjugates. At pH lower than 8.3 (cysteinyl thiol pKa), cysteine lowered alkaline CGA-lysine greening by reducing CGA o-quinones to CGA diphenols; cysteine lowers CGA-lysine greening by preferentially forming cysteinyl-CGA conjugates which also contributes to antioxidant capacity. Visible greening was fully inhibited with a cysteine: 
lysine 1:1 molar ratio in pH 8 and 9 CGA: Lys: Cys solutions, indicating that cysteinyl thiol was a stronger nucleophile than $\varepsilon$-lysyl amine to react with CGA o-quinones. Cysteine had a greater effect to promote browning while lowering $\mathrm{pH}$ was more effective on anti-greening (Table 4).

Lowering $\mathrm{pH}$ from 9 to 8, retained up to 50\% higher antioxidant capacity without cysteine; with addition of cysteine, differences in given by lowered $\mathrm{pH}$ diminished because formation of cysteinyl CGA conjugates compensated lost antioxidant capacity by CGA diphenols. The individual and combined effects of cysteine concentration and $\mathrm{pH}$ were all significant to enhance reducing than radical scavenger capacity (Table 4).

\section{Recommendations for future studies}

Addition of cysteine during alkaline $\mathrm{pH}$ protein extraction from sunflower meal is recommended to determine an optimal cysteine-pH combination for industrial alkaline protein extraction from sunflower meal that is not de-phenolized with organic solventwater mixture.

Serine and threonine are two amino acids with nucleophilic side chain, which do not form green TBA derivatives with CGA quinones at alkaline pH (Bongartz et al., 2016). Addition of serine and threonine to inhibit CGA-lysine greening are also recommended. Inhibition of serine and threonine on CGA quinone induced greening (Bongartz et al., 2016) would indicate that any nucleophile stronger than $\alpha$-amines can competitively inhibit formation of green TBA derivatives, which will complement knowledge of CGA-protein interactions and benefit future development of CGA-rich sunflower protein products.

Experiments of di-thiol compounds, such as dithiothreitol, and a different mono- 
thiol compound, glutathione, are also recommended. Comparison experiments of cysteine and glutathione, ubiquitous in plant sources such as mushrooms, added into CGA-lysine mixtures at the same concentrations are necessary to generalize the cysteine anti-greening mechanism to other thiol-containing food ingredients, as preliminary results show similar decolorization (Figure 17).

A) Greening in pH 9 buffered CGA: lysine: glutathione solutions incubated at ambient temperature for $24 \mathrm{~h}$

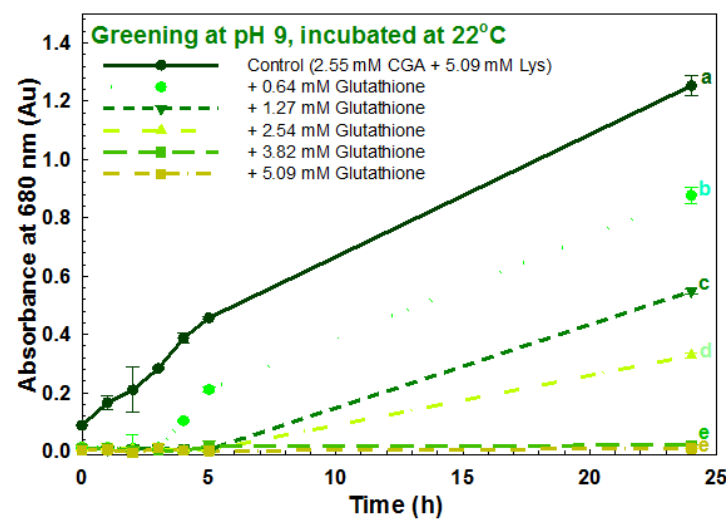

C) Browning in pH 9 buffered CGA: lysine: glutathione solutions incubated at ambient temperature for $24 \mathrm{~h}$

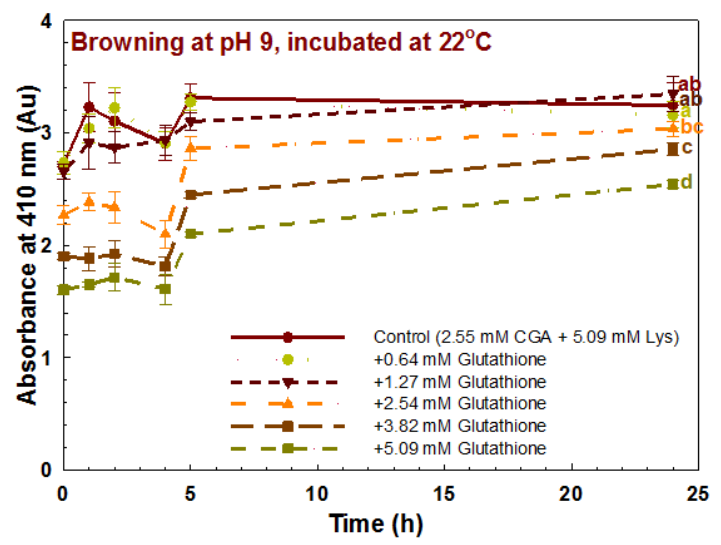

B) Greening in pH 8 buffered CGA: lysine: glutathione solutions incubated at ambient temperature for $24 \mathrm{~h}$

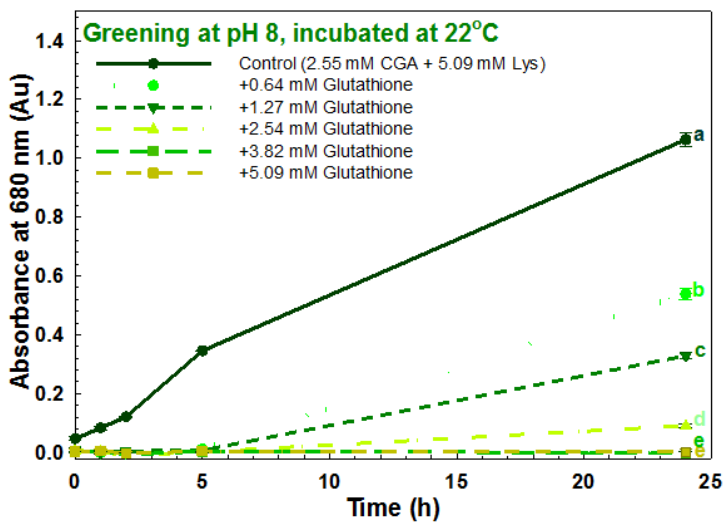

D) Browning in pH 8 buffered CGA: lysine: glutathione solutions incubated at ambient temperature for $24 \mathrm{~h}$

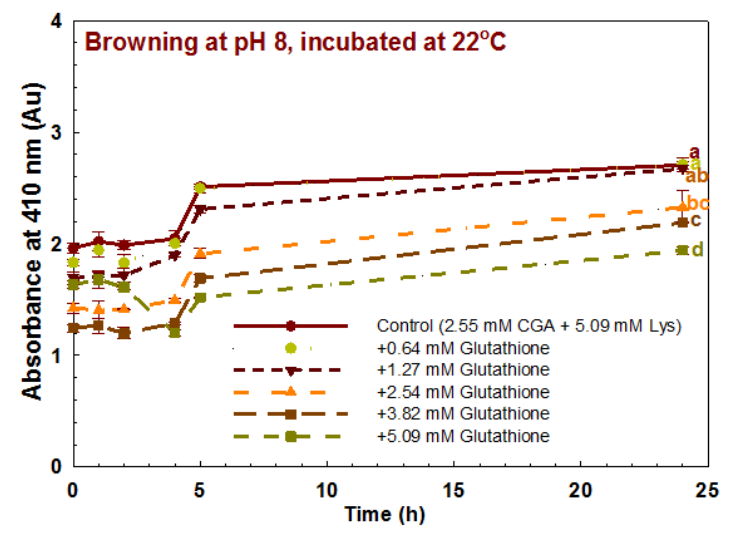

Figure 17 Greening measured by absorbance at $680 \mathrm{~nm}$ (A, B), browning by absorbance at $410 \mathrm{~nm}$ (C, D) of pH 8 and 9 buffered chlorogenic acid (CGA): lysine: glutathione solutions incubated at ambient temperature for $24 \mathrm{~h}$ when glutathione increased from 0 to $5.091 \mathrm{mM}$. 
Glutathione may be a less effective anti-greening compound compared to cysteine as shown in Figure 11, consistent with Kuijpers et al. (2012) found that cysteine had a higher tendency to form thiol-conjugates with CGA o-quinones than glutathione. Comparing dithiothreitol and cysteine in terms of anti-greening can determine the difference and correlation between inhibited greening and sulfhydryl concentrations.

\section{References}

Ajandouz, E. H., \& Puigserver, A. (1999). Nonenzymatic browning reaction of essential amino acids: effect of $\mathrm{pH}$ on caramelization and maillard reaction kinetics. Journal of agricultural and food chemistry(5), 1786-1793.

Altunkaya, A., \& Gökmen, V. (2009). Effect of various anti-browning agents on phenolic compounds profile of fresh lettuce (L. sativa). Food Chemistry, 117, 122-126. doi:10.1016/j.foodchem.2009.03.085

Ashoor, S. H., \& Zent, J. B. (1984). Maillard browning of common amino acids and sugars. Journal of Food Science (USA)(4), 1206-1207.

Atonfack, J. T., Ataman, Z. A., \& Were, L. M. (2019). Acidulant Effect on Greening, Reducing Capacity, and Tryptophan Fluorescence of Sunflower Butter Cookie Dough During Refrigerated Storage. Journal of the Science of Food and Agriculture, 99(5), 2186-2193.

Bassil, D., Makris, D. P., \& Kefalas, P. (2005). Oxidation of caffeic acid in the presence of Lcysteine: isolation of 2-S-cysteinylcaffeic acid and evaluation of its antioxidant properties. Food research international(4), 395-402.

Billaud, C., Roux, E. m., Brun-Mérimee, S., Maraschin, C., \& Nicolas, J. (2003). Inhibitory effect of unheated and heated D-glucose, D-fructose and L-cysteine solutions and Maillard reaction product model systems on polyphenoloxidase from apple. I. Enzymatic browning and enzyme activity inhibition using spectrophotometric and polarographic methods. Food chemistry, 81(1), 35-50. doi:10.1016/S0308-8146(02)00376-X

Bittner, S. (2006). When quinones meet amino acids: chemical, physical and biological consequences. Amino Acids, 30(3), 205-224.

Bongartz, V., Brandt, L., Gehrmann, M. L., Zimmermann, B. F., Schulze-Kaysers, N., \& Schieber, A. (2016). Evidence for the Formation of Benzacridine Derivatives in AlkalineTreated Sunflower Meal and Model Solutions. Molecules, 21(1), 91-99.

Budryn, G., \& Rachwal-Rosiak, D. (2013). Interactions of Hydroxycinnamic Acids with Proteins and Their Technological and Nutritional Implications. Food Reviews International, 29(3), 217-230. doi:10.1080/87559129.2012.751545

Cao, Y., \& Xiong, Y. L. (2015). Chlorogenic acid-mediated gel formation of oxidatively stressed myofibrillar protein. Food Chemistry, 180, 235-243. doi:10.1016/j.foodchem.2015.02.036

Chapin, M. M., Rochette, L. M., Annest, J. L., Haileyesus, T., Conner, K. A., \& Smith, G. A. (2013). Nonfatal choking on food among children 14 years or younger in the United States, 2001-2009. Pediatrics(2), 275-284.

Cilliers, J. J. L., \& Singleton, V. L. (1989). Nonenzymic autoxidative phenolic browning reactions in a caffeic acid model system. Journal of agricultural and food chemistry, 890896. 
Cilliers, J. J. L., \& Singleton, V. L. (1990). Caffeic acid autoxidation and the effects of thiols. Journal of agricultural and food chemistry (USA)(9), 1789-1796

Cilliers, J. J. L., \& Singleton, V. L. (1991). Characterization of the products of nonenzymic autoxidative phenolic reactions in a caffeic acid model system. Journal of agricultural and food chemistry (USA)(7), 1298-1303.

Eardley, M. (2018). Capitalize on the Growing Trend of Plant-Based Proteins. Winsight Grocery Business. Retrieved from https://www.winsightgrocerybusiness.com/retailfoodservice/capitalize-growing-trend-plant-based-proteins

Eric, K., Raymond, L. V., Abbas, S., Song, S., Zhang, Y., Masamba, K., \& Zhang, X. (2014). Temperature and cysteine addition effect on formation of sunflower hydrolysate Maillard reaction products and corresponding influence on sensory characteristics assessed by partial least square regression. Food Research International, 57, 242-258. doi:10.1016/j.foodres.2014.01.030

Eric, K., Raymond, L. V., Huang, M., Cheserek, M. J., Hayat, K., Savio, N. D., . . Zhang, X. (2013). Sensory attributes and antioxidant capacity of Maillard reaction products derived from xylose, cysteine and sunflower protein hydrolysate model system. Food Research International, 54, 1437-1447. doi:10.1016/j.foodres.2013.09.034

FDA. (2019). 2018 Recalls, Market Withdraws, and Safety Alerts. Retrieved from https://www.fda.gov/safety/archive-recalls-market-withdrawals-safety-alerts/2018recalls-market-withdrawals-safety-alerts

Ferrer, B. (Producer). (2019). Givaudan unveils "most promising new plant-based proteins” in collaboration with UC Berkeley. Retrieved from https://www.foodingredientsfirst.com/news/givaudan-unveils-most-promising-new-plantbased-proteins-in-collaboration-with-uc-berkeley.html

Fujimoto, A., Inai, M., \& Masuda, T. (2013). Chemical evidence for the synergistic effect of a cysteinyl thiol on the antioxidant activity of caffeic and dihydrocaffeic esters. Food Chemistry, 138, 1483-1492. doi:10.1016/j.foodchem.2012.11.073

Gabriel, A. A., Joe, A. V., \& Patrick, E. D. (2014). Folin-Ciocalteau Reagent for Polyphenolic Assay. International Journal of Food Science, Nutrition and Dietetics (IJFS)(8), 147156.

Gonzales-Perez, S., Merck, K. B., Vereijken, J. M., Koningsveld, v., G.A., \& Gruppen, H., Voragen, A.G.J. (2002). Isolation and characterization of undenatured chlorogenic acid free sunflower (Helianthus annuus) Proteins. Journal of Agricultural and Food Chemistry, 50, 1713-1719.

Hartman, G. (Producer). (2019). Saying No to Meat. What's Behind the Rising Interest in PlantBased Protein? Newsletter.

Hayase, F., Kim, S. B., \& Kato, H. (1984). Decolorization and degradation products of the melanoidins by hydrogen peroxide. Agricultural and Biological Chemistry (Japan)(11), 2711-2717.

Hwang, S. H., Zuo, G., Wang, Z., \& Lim, S. S. (2018). Novel aldose reductase inhibitory and antioxidant chlorogenic acid derivatives obtained by heat treatment of chlorogenic acid and amino acids. Food Chemistry, 266, 449-457. doi:10.1016/j.foodchem.2018.06.053

Iacomino, M., Weber, F., Gleichenhagen, M., Pistorio, V., Panzella, L., Pizzo, E., . . . Napolitano, A. (2017). Stable Benzacridine Pigments by Oxidative Coupling of Chlorogenic Acid with Amino Acids and Proteins: Toward Natural Product-Based Green Food Coloring. 65, 6519-6528. doi:10.1021/acs.jafc.7b00999

Indrani, D., Prabhasankar, P., Rajiv, J., \& Rao, G. V. (2003). Scanning Electron Microscopy, Rheological Characteristics, and Bread-Baking Performance of Wheat-Flour Dough as Affected by Enzymes. Journal of Food Science(9), 2804-2812.

Jackson, H., \& Kendal, L. P. (1949). The Oxidation of Catechol and Homocatechol by Tyrosinase in the Presence of Amino-acids. Journal of Biochemistry, 44, 477-487. 
Kalaras, M. D., Richie, J. P., Calcagnotto, A., \& Beelman, R. B. (2017). Mushrooms: A rich source of the antioxidants ergothioneine and glutathione. 233, 429-433. doi:10.1016/j.foodchem.2017.04.109

Kuijpers, T. F. M. (2013). Inhibition of tyrosinase-induced enzymatic browning by sulfite and natural alternatives. (PHD). Wageningen University, Wageningen University, Wageningen, NL. (978-94-6173-668-0)

Kuijpers, T. F. M., Narvaez Cuenca, C. E., Vincken, J. P., Verloop, J. W., Berkel, v., W.J.H., \& Gruppen, H. (2012). Inhibition of Enzymatic Browning of Chlorogenic Acid by SulfurContaining Compounds. 3507-3514.

Kumar, N., Khatkar, B. S., \& Kaushik, R. (2013). Effect of reducing agents on wheat gluten and quality characteristics of flour and cookies. Annals of the University Dunarea de Jos of Galati Fascicle VI -- Food Technology, 37(2), 68-81.

Lan, X., Liu, P., Xia, S., Jia, C., Mukunzi, D., Zhang, X., . . Xiao, Z. (2010). Temperature effect on the non-volatile compounds of Maillard reaction products derived from xylosesoybean peptide system: Further insights into thermal degradation and cross-linking. Food chemistry(4), 967-972. doi:10.1016/j.foodchem.2009.11.033

Lester, M. R. (1995). Sulfite sensitivity: significance in human health. Journal of the American College of Nutrition (USA)(3), 229-235.

Li, W.-W., Wolfgang, H., \& Jurgen, H. (2005). Site-specific binding of quinones to proteins through thiol addition and addition-elimination reactions. Journal of the American Chemical Society(17), 6140-6141.

Liang, S., Were, L., \& Hanh Lan, T. (2018). Lowering greening of cookies made from sunflower butter using acidic ingredients and effect on reducing capacity, tryptophan and protein oxidation. Food Chemistry, 252, 318-326.

Liang, S., \& Were, L. M. (2018a). Chlorogenic acid induced colored reactions and their effect on carbonyls, phenolic content, and antioxidant capacity in sunflower butter cookies. LWTFood Science and Technology, 87, 16-22. doi:10.1016/j.lwt.2017.08.069

Liang, S., \& Were, L. M. (2018b). Chlorogenic acid oxidation-induced greening of sunflower butter cookies as a function of different sweeteners and storage conditions. Food Chemistry, 241, 135-142.

Lund, M. N., Heinonen, M., Baron, C. P., \& Estevez, M. (2011). Protein oxidation in muscle foods: A review. Molecular Nutrition \& Food Research, 55, 83-95. doi:10.1002/mnfr.201000453

Lund, M. N., \& Ray, C. A. (2017). Control of Maillard Reactions in Foods: Strategies and Chemical Mechanisms. Journal of Agricultural and Food Chemistry, 65, 4537-4552. doi:10.1021/acs.jafc.7b00882

Maegawa, Y., Sugino, K., \& Sakurai, H. (2007). Identification of free radical species derived from caffeic acid and related polyphenols. Free Radical Research, 41(1), 110-119. doi:10.1080/10715760600943892

Miura, Y., Inai, M., Honda, S., Masuda, A., \& Masuda, T. (2014). Reducing Effects of Polyphenols on Metmyoglobin and the in Vitro Regeneration of Bright Meat Color by Polyphenols in the Presence of Cysteine. Journal of agricultural and food chemistry(39), 9472-9478.

Murata, M., Sugiura, M., Sonokawa, Y., Shimamura, T., \& Homma, S. (2002). Properties of chlorogenic acid quinone: Relationship between browning and the formation of hydrogen peroxide from a quinone solution. Bioscience, Biotechnology, and Biochemistry (Japan)(12), 2525-2530.

Namiki, M., Yabuta, G., Koizumi, Y., \& Yano, M. (2001). Development of free radical products during the greening reaction of caffeic acid esters (or chlorogenic acid) and a primary amino compound. Bioscience, Biotechnology, and Biochemistry (Japan)(10), 2131-2136. 
Ningjian, L., \& David, D. K. (2015). Role of Chlorogenic Acids in Controlling Oxidative and Inflammatory Stress Conditions. Nutrients, 8(1), 16-35. doi:10.3390/nu8010016

Ooms, N., Jansens, K. J. A., Pareyt, B., Reyniers, S., Brijs, K., \& Delcour, J. A. (2018). The impact of disulfide bond dynamics in wheat gluten protein on the development of fermented pastry crumb. Food Chemistry, 242, 68-74. doi:10.1016/j.foodchem.2017.09.007

Panera, B. (2018). The No No List, Panera bread. Retrieved from https://www.panerabread.com/panerabread/documents/panera-no-no-list-05-2015.pdf

Penalver, M. J., Rodriguez-Lopez, J. N., Garcia-Molina, F., Garcia-Canovas, F., \& Tudela, J. (2002). Method for the determination of molar absorptivities of thiol adducts formed from diphenolic substrates of polyphenol oxidase. Analytical Biochemistry(2), 180-185.

Pierpoint, W. S. (1966). The enzymic oxidation of chlorogenic acid and some reactions of the quinone produced. Biochem J, 2(98), 567-580.

Pierpoint, W. S. (1969). O-Quinones formed in plant extracts; their reactions with amino acids and peptides. Bio-chemical journal(112), 609-616.

Prigent, S. V. E., Voragen, A. G. J., Li, F., Visser, A. J. W. G., Koningsveld, v., G.A., \& Gruppen, H. (2008). Covalent interactions between amino acid side chains and oxidation products of caffeoylquinic acid (chlorogenic acid). Journal of the Science of Food and Agriculture, 88, 1748-1754.

Radiana Maria, T. B., Ciprian Nicolae, P., \& Stela, P. (2013). Assessment of the E920 additive (L-cysteine) in relation to some problems of modern food industry. Scientific Papers Series : Management, Economic Engineering in Agriculture and Rural Development, 13(1), 413-418.

Rawel, H. M., \& Rohn, S. (2010). Nature of hydroxycinnamate-protein interactions. Phytochemistry Reviews(1), 93-109.

Richard, F. C., Goupy, P. M., Nicolas, J. J., Lacombe, J. M., \& Pavia, A. A. (1991). Cysteine as an inhibitor of enzymatic browning. 1. Isolation and characterization of addition compounds formed during oxidation of phenolics by apple polyphenol oxidase. Journal of agricultural and food chemistry (USA)(5), 841-849.

Richard-Forget, F. C., Goupy, P. M., \& Nicolas, J. J. (1992). Cysteine as an inhibitor of enzymatic browning. 2. Kinetic studies. Journal of agricultural and food chemistry (USA), 40(11), 2108-2113.

Rogers, A., Lan, H., Vu, P., \& Were, L. (2018). Greening in sunflower butter cookies as a function of egg replacers and baking temperature. In (Vol. 55, pp. 1478-1488).

Rong, T. (2010). Chemistry and Biochemistry of Dietary Polyphenols. Nutrients, Vol 2, Iss 12, Pp 1231-1246 (2010)(12), 1231. doi:10.3390/nu2121231

SAS, I. I. (2014). Statistical Analysis Software (Version SAS/ACCESS® 9.3). Cary, NC, USA: SAS Institute Inc. Retrieved from https://www.sas.com/en_us/home.html

Schilling, S., Sigolotto, C. I., Carle, R., \& Schieber, A. (2008). Characterization of covalent addition products of chlorogenic acid quinone with amino acid derivatives in model systems and apple juice by high-performance liquid chromatography/electrospray ionization tandem mass spectrometry. Rapid Communications In Mass Spectrometry(4), 441-448.

Schlichtherle-Cerny, H., \& Amado, R. (2002). Analysis of Taste-Active Compounds in an Enzymatic Hydrolysate of Deamidated Wheat Gluten. Journal of Agricultural and Food Chemistry(6), 1515-1528.

Snell, J. M., \& Weissberger, A. (1939). Reaction of thiol compounds with quinones. Journal of the American Chemical Society, 61, 450-453.

Timbo, B., Koehler, K. M., Wolyniak, C., \& Klontz, K. C. (2004). Sulfites--a Food and Drug Administration review of recalls and reported adverse events. Journal of food protection(8). 
Tosovic, J., Markovic, S., Markovic, J. M. D., Mojovic, M., \& Milenkovic, D. (2017). Antioxidative mechanisms in chlorogenic acid. Food Chemistry, 237, 390-398. doi:10.1016/j.foodchem.2017.05.080

Whole Foods Market, I. L. P. (2018). Whole Foods Market's Unacceptable Ingredients \& Food. Retrieved from https://www.wholefoodsmarket.com/values-matter/unacceptableingredients-food

Yabuta, G., Koizumi, Y., Namiki, K., Hida, M., \& Namiki, M. (2001). Structure of green pigment formed by the reaction of caffeic acid esters (or chlorogenic acid) with a primary amino compound. Bioscience, Biotechnology, and Biochemistry (Japan), 65(10), 2121-2130.

Yu, L., Nanguet, A.-L., \& Beta, T. (2013). Comparison of Antioxidant Properties of Refined and Whole Wheat Flour and Bread. Antioxidants(4), 370-383. doi:10.3390/antiox2040370

Zeng, X., Bai, W., Zhu, X., \& Dong, H. (2017). Browning Intensity and Taste Change Analysis of Chicken Protein-Sugar Maillard Reaction System with Antioxidants and Different Drying Processes. Journal of Food Processing \& Preservation, 41(2), 1-7. doi:10.1111/jfpp.13117

Zhang, Z., Elfalleh, W., He, S., Tang, M., Zhao, J., Wu, Z., . . . Sun, H. (2018). Heating and cysteine effect on physicochemical and flavor properties of soybean peptide Maillard reaction products. International Journal of Biological Macromolecules, 120(Part B), 2137-2146. doi:10.1016/j.ijbiomac.2018.09.082 


\section{APPENDIX: Preliminary studies with cookies}

\subsection{Cysteine as a dough conditioner in baked products}

L-cysteine/L-cysteine $\mathrm{HCl}$ is affirmed as a generally recognized as safe (GRAS) ingredient by the U.S. Food and Drug Administration (21 CFR. 184. 1271/ 1272). European Food Safety Authority permits the use of L-cysteine (E920) at levels indicated by quantum satis under regulation No 1129/2011. “Quantum satis” indicates that no maximum level is specified. Baked foods are category of food products with added cysteine.

Biscuits/ cookies/ crackers caused 4.6\% non-fatal choking incidents for 0 to 4 years old children, and was classified as one of the top three choking inducing food for children less than 12 months of age (Chapin et al., 2013). Dough conditioners such as cysteine and glutathione in industrial manufactured cookies (Indrani, Prabhasankar, Rajiv, \& Rao, 2003) can prevent cookie cracking and thus reduce the risk of choking. Cysteine prevents biscuits, cookies and crackers' cracking in the baking industry because the reducing capacity of its thiol group can dissociate disulfide bonds of gluten network (Ooms et al., 2018). This gluten network formed during mixing and kneading of wheat dough can be undesirable in cookies because it leads to cracking and increased choking risk for young children (Radiana Maria et al., 2013).

Three commonly used reducing agents in baked goods include L-cysteine, sulfites (sodium bisulfites and sodium metabisulfites), and inactivated yeast, a natural source of glutathione. Glutathione is also found high content in Pioppini (Agrocybe aegerita) mushrooms (Kalaras, Richie, Calcagnotto, \& Beelman, 2017). Among these three reducing agents, reported sensitivity of sulfites for up to 3\% adults with asthma (Lester, 
1995) has led to limited use of sulfites in U.S.A. From 1996 to 1999, 55\% of Class I food recalls (Timbo, Koehler, Wolyniak, \& Klontz, 2004) and 13 food product recalls in 2018 were due to undeclared sulfites (FDA, 2019). Comparing glutathione and cysteine, Kuijpers et al. (2012) found that L-cysteine had a higher tendency to form adducts with o-quinones than glutathione while dough treated with glutathione had less gluten recovery than dough treated with cysteine (Kumar, Khatkar, \& Kaushik, 2013), indicating glutathionyl thiol was more prone to reduce disulfide of gluten network while cysteinyl thiol was more prone to trap CGA $o$-quinones. It was the goal to investigate cysteine’s anti-greening effects on sunflower butter cookies at FDA approved levels.

\subsection{Formulation, baking parameters and L-cysteine addition methods of sunflower butter cookies}

A total mass of $760.0 \mathrm{~g}$ cookie dough was prepared for each treatment, which was composed of 303.75 g Gold Medal® all-purpose baking flour/ UPC: 0001600010610 (39.97\% by weight), 182.25 g Once Again ${ }^{\circledR}$ un-sweetened and un-salted sunflower seeds butter (Once Again ${ }^{\circledR}$, Nunda, NY)/UPC: 044082530413 (23.98\% by weight), 153.0 g Trader Joe’s® 100\% pure U.S. Grade A dark maple syrup/ UPC: 700736111697 (20.13\% by weight), $97.50 \mathrm{~g}$ egg (12.83\% by weight), $4.50 \mathrm{~g}$ of Trader Joe’s ${ }^{\circledR}$ baking soda/ UPC: 085239991466, Trader Joe’s® alcohol-free pure vanilla flavor/UPC: 008281230000 and Tradewinds ${ }^{\circledR}$ salt/ UPC: 041512005275 (0.59\% by weight). L-cysteine was added by dissolving L-cysteine-HCl in $10 \mathrm{~mL}$ DI water before mixing with other ingredients in order to achieve an even distribution in cookie doughs. $\mathrm{pH}$ of L-cysteine solutions was not adjusted.

Cookie dough pieces were obtained using a $5 \mathrm{~cm}$ diameter Kispowwin ${ }^{\circledR}$ round 
stainless steel cookie cutter, and thickness of each dough piece was controlled using a Milimoli ${ }^{\circledR}$ adjustable stainless steel rolling pin with a $0.635 \mathrm{~cm}(0.25$ in.) thickness controlling ring. Dough was baked in a Hobart Hec 20 convection oven (Troy. OH. USA) at $149^{\circ} \mathrm{C}\left(300^{\circ} \mathrm{F}\right)$ for $7 \mathrm{~min}$. After baking, cookies were stored in open air at ambient temperature before color analysis.

\subsection{Color intensity analysis}

Upper surface and internal (transection) color analysis of sunflower butter cookies included measuring Hunter $\mathrm{L}^{*}$ [darkness to lightness $(0-100)$ ], $\mathrm{a}^{*}$ [greenness $\left(-\mathrm{a}^{*}\right)$ to redness $\left(\mathrm{a}^{*}\right)$ ], and $\mathrm{b}^{*}$ [blueness $\left(-\mathrm{b}^{*}\right)$ to yellowness $\left(\mathrm{b}^{*}\right)$ ] values using a spectrophotometer (CM-2500d, Konica Minolta, Inc. Japan) and \% greening using an image analyzing camera (CV-X422A) with associated software CA-H1DB Vision Database Ver 1.2 (Keyence America, Corp. CA. USA) . Upper surface and internal color changes were tracked after 1, 4, 8, and $24 \mathrm{~h}$ of baking, and analyzed using SpectraMagic NX color data software. Surface and internal percent greening were calculated by dividing green area by the whole selected cookie area.

\section{Cysteine effects on greening and browning of sunflower butter cookies}

Sunflower butter cookies with addition of cysteine as an anti-greening approach

were studied as a CGA-rich food model. As cysteine concentration increased from $0 \mathrm{mM}$ to $3.72 \mathrm{mM}$ (Figure 18, Table 8), internal \% greening increased as a function of storage time, which was consistent with Liang \& Were (2018b) that formation of green TBA in sunflower butter cookies was a function of storage time. 
Hunter $\mathrm{L}^{*}$, $\mathrm{a}^{*}$, or $\mathrm{b}^{*}$ values (Table 7) were consistent with \% greening (Figure Figure 18 Internal greening (\%) of

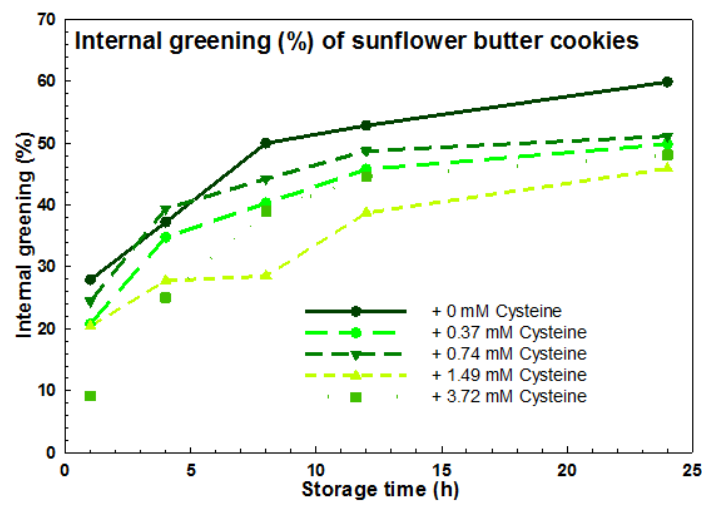
sunflower butter cookies as a function of storage time during uncorvered storage at ambient temperature when L-cysteine was increased from 0 to $3.72 \mathrm{mM}$.

18). Photos of cookies (Table 8) also showed that internal greening accumulated during $24 \mathrm{~h}$ of un-sealed storage at ambient temperature. Cysteine concentration did not affect internal \%greening and Hunter L*, a*, or b* values of sunflower butter cookies as Internal Hunter $\mathrm{L}^{*}, \mathrm{a}^{*}$ and $\mathrm{b} *$ values were a function of storage time $(\mathrm{F}=84.24$, $\mathrm{p}<0.0001$ for $\mathrm{L}^{*} ; \mathrm{F}=87.54, \mathrm{p}<0.0001$ for $\mathrm{a}^{*} ; \mathrm{F}=9.88, \mathrm{p}<0.001$ for $\left.\mathrm{b}^{*}\right)$. Hunter $\mathrm{a}^{*}$ and b* values decreased corresponding to greener cookies over time (Table 8). Increased Hunter $L^{*}$ value due to longer storage time simultaneously indicated that CGA quinones and proteins primarily reacted to form green TBA derivatives but not melanoidins by Maillard reaction, because formation of melanoidins would led to darkness and thus decreased $L^{*}$ values. 
Table 7 Hunter L*, a*, or b* values of sunflower butter cookies internal transections as a function of storage time when L-cysteine was increased from 0 to $3.72 \mathrm{mM}$.

\begin{tabular}{|c|c|c|c|c|c|}
\hline L-cysteine (mM) & $1 \mathrm{~h}$ & $4 \mathrm{~h}$ & $8 \mathrm{~h}$ & $12 \mathrm{~h}$ & $24 \mathrm{~h}$ \\
\hline \multicolumn{6}{|c|}{$\mathbf{L}^{*}$} \\
\hline 0 & 12.59 & 33.58 & 33.73 & 30.50 & 27.97 \\
\hline 0.37 & 13.22 & 35.15 & 31.91 & 32.10 & 30.20 \\
\hline 0.74 & 11.03 & 35.63 & 32.11 & 33.23 & 29.22 \\
\hline 1.49 & 13.61 & 37.97 & 33.07 & 31.84 & 30.40 \\
\hline 3.72 & 11.72 & 37.62 & 36.82 & 33.51 & 31.54 \\
\hline \multicolumn{6}{|c|}{$\mathbf{a}^{*}$} \\
\hline 0 & -4.845 & -13.16 & -13.45 & -12.08 & -11.02 \\
\hline 0.37 & -5.325 & -12.55 & -12.37 & -12.49 & -11.59 \\
\hline 0.74 & -5.500 & -12.83 & -12.19 & -12.60 & -11.32 \\
\hline 1.49 & -5.865 & -12.69 & -12.65 & -12.09 & -11.68 \\
\hline 3.72 & -4.965 & -10.77 & -11.23 & -12.23 & -11.19 \\
\hline \multicolumn{6}{|c|}{ b* } \\
\hline 0 & 12.12 & 12.51 & 9.91 & 7.656 & 4.891 \\
\hline 0.37 & 11.28 & 15.78 & 12.18 & 10.70 & 7.814 \\
\hline 0.74 & 10.12 & 14.91 & 12.89 & 9.819 & 6.635 \\
\hline 1.49 & 10.98 & 17.32 & 13.73 & 11.40 & 7.624 \\
\hline 3.72 & 10.99 & 18.05 & 14.23 & 11.58 & 8.221 \\
\hline
\end{tabular}

Internal color changes over time were consistent with findings in alkaline CGA: Lys: Cys solutions (Figure 12) that primary amines would nucleophilically substituted CGA quinones to form amine-CGA hydroquinones, the predominant green TBA precursor. Cysteine could hinder formation of Schiff bases (Zeng et al., 2017; Zhang et al., 2018) but promoted formation of CGA dimers (Figure 13). In addition, cysteine would act as a reducing agent to break down the gluten network of cookie dough. Therefore, no detectable decolorization at higher cysteine concentration might be attributed to limited availability of cysteinyl thiol to trap highly reactive CGA $o$-quinones before they react with amines. 
Table 8 Progression of surface and internal color in sunflower butter cookies as a function of time and L-cysteine concentration during uncovered storage at ambient temperature.

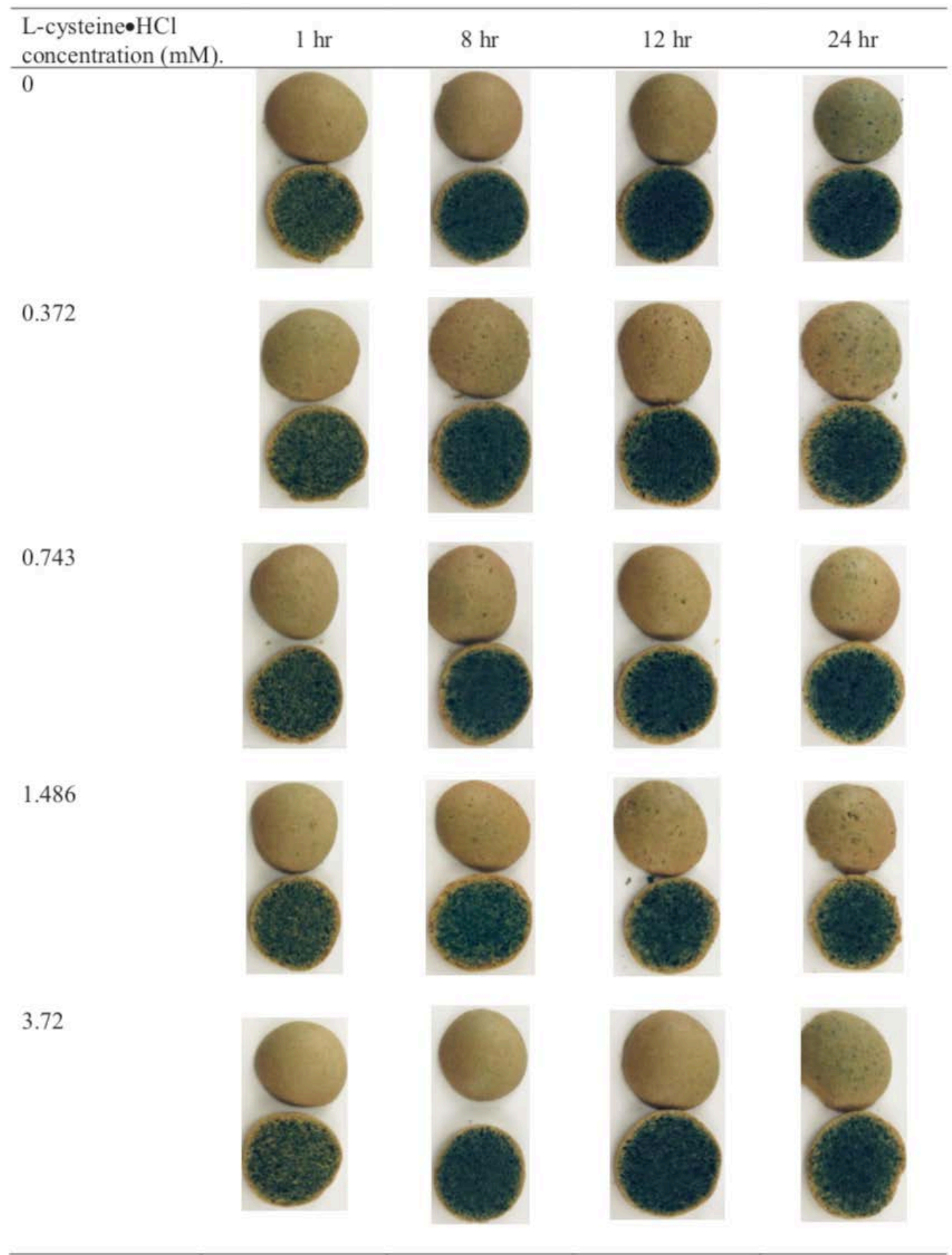

The use of L-cysteine in USA is limited in baked products to 90ppm. It is also identified as one of the unacceptable ingredients for food products sold by restaurants and 
grocery chains such as Panera and Whole Foods Market, due to the negative perception of cysteine amongst some customers (Panera, 2018; Radiana Maria et al., 2013; Whole Foods Market, 2018). However, there is no usage limit of L-cysteine in Europe and Australia (Radiana Maria et al., 2013). L-cysteine use can be more widely used in CGAcontaining food products in Europe and Australia markets where there is less negative consumer perception, or in niche markets in USA to provide a potential anti-greening solution in CGA-containing products. 


\section{MANUSCRIPT Title}

2 Cysteine's effects on chlorogenic acid quinone induced greening and browning:

3 mechanism and effect on antioxidant reducing capacity

4

\section{Author names and affiliations}

$6 \quad$ Yundi LIANG, yuliang@chapman.edu

$7 \quad$ Lilian WERE, were@chapman.edu

8

9 Food Science Program, Schmid College of Science and Technology, Chapman

10 University,

11 One University Drive, Orange CA 92866, USA

12

\section{Corresponding Author}

14 Lilian Were, Ph. D. were@chapman.edu

15 Phone: 714-744-7895

16 Keck Center for Science and Engineering 256

17 One University Drive, Orange, CA 92866 
19 Formation of green trihydroxy benzacridine (TBA) derivatives when chlorogenic acid

20 (CGA) quinones and amino acids react can be unappealing for some consumers. Cysteine

21 was studied as an anti-greening strategy, because cysteine-CGA conjugates are colorless.

22 Buffered 2.55 mM CGA: 5.09 mM lysine: (0- 5.09) mM cysteine solutions at pH 8 and 9

23 were prepared and incubated for a maximum of $48 \mathrm{~h}$ at room temperature. Color intensity

24 and conjugate formation was monitored spectrophotometrically and by HPLC and LC-

25 MS respectively, while antioxidant capacity was measured by Folin-Ciolcateau and

26 Trolox equivalent antioxidant capacity assays. Green TBA formation was promoted at

27 higher $\mathrm{pH}$, and inhibited as cysteine concentration increased. Concentration-dependent

28 cysteine inhibition of CGA-lysine greening was by redox diphenol regeneration and

29 formation of cysteinyl-CGA conjugates, which also contributed to antioxidant capacity.

$30 \mathrm{pH}$ had a greater effect on antioxidant capacity than cysteine. Results provided a potential

31 anti-greening approach for alkaline CGA quinone-amine greening.

33 Keywords: Antioxidant capacity; browning; chlorogenic acid quinones; cysteine;

34 greening

35 Chemical Compounds studied in this article:

36 Chlorogenic acid (PubChem CID:1794427)

37 L-cysteine (PubChem CID: 5862)

38 L-lysine (PubChem CID: 5962) 


\section{1. Introduction}

2 Chlorogenic acid (CGA) contributes to the color, aroma and taste profile of foods.

3 Intense greening can however occur at alkaline $\mathrm{pH}$ when CGA ortho-quinones react with

4 primary amino acids without sulfhydryl, $\alpha$-hydroxyl group or an indole ring as side chain

5 to form green trihydroxy benzacridine (TBA) derivatives (Bongartz et al., 2016).

6 Chlorogenic acid $o$-quinones and primary amine groups are the primary substrates for this

7 TBA greening (Yabuta et al., 2001). Under high pH conditions, such as alkaline

8 sunflower protein isolate extraction, primary amines nucleophilically attack CGA o-

9 quinones to form N-quinonyl amino acid conjugates, followed with nucleophilic

10 cyclization and loss of a water molecule to form green pigmented trihydroxy

11 benzacridine (TBA) derivatives (Bongartz et al., 2016; Namiki et al., 2001; Rawel \&

12 Rohn, 2010; Yabuta et al., 2001).

13 Incorporating acidic ingredients such as honey, yogurts, and dough acidulants, into

14 cookie dough formulation was shown to inhibit CGA quinone induced greening by

15 lowering pH (Atonfack et al., 2019; Liang et al., 2018; Liang \& Were, 2018b; Rogers,

16 Lan, Vu, \& Were, 2018b), because acidic $\mathrm{pH}$ prevents deprotonated phenolic ions from

17 oxidizing to form CGA quinones (Cilliers \& Singleton, 1989). However, up to 51\%

18 intersectional greening occurred within $24 \mathrm{~h}$ post baking when sunflower butter cookies

19 formulated with acidic dairy ingredients (Liang et al., 2018). In addition to potential sour

20 taste due to acidic dairy ingredients, no anti-greening method was reported to fully inhibit

21 greening.

22 Chlorogenic acid quinone-amino acid induced greening does not occur when CGA o-

23 quinones react with primary amino acids having a nucleophile other than an amine, or an 
24 indole ring as their side groups. Bongartz et al. (2016) reported the formation of colorless

25 S-quinonyl amino acid conjugates formed by CGA and cysteine at $\mathrm{pH}$ 9. Unlike the thiol-

26 ether sulfur group of methionine, the cysteinyl thiol can ionize to a highly nucleophilic

27 thiolate above its $\mathrm{pKa}$ of 8.3. When thiol groups react with quinones at alkaline $\mathrm{pH}$, a

28 covalent attachment of thiolates to quinones forms colorless mono- or di-thiol-substituted

29 quinone conjugates (Li et al., 2005; Schilling et al., 2008; Snell \& Weissberger, 1939).

30 Cysteine can also inhibit Maillard browning by hindering imine formation and

31 polymerization because thiol groups are more nucleophilic than amines. Zhang et al.

32 (2018) reported that thiol groups are more prone to react with carbonyls which hinders

33 imine formation and thus cysteine lowers melanoidin formation in the Maillard reaction.

34 Eric et al. (2013) also found that adding cysteine to a mixture of sunflower protein isolate

35 and xylose decreased melanoidins, as cysteine competitively consumed CGA carbonyls.

36 Decreased formation of melanoidins during Maillard reaction could also be a result of

37 cysteine inhibited crosslinking of low molecular weight (MW) peptides and promoted

38 degradation of high MW peptides, suppressing the formation of high MW Maillard

39 reaction products (MRPs) including melanoidins (Eric et al., 2014). To evaluate whether

40 cysteine is a potential anti-greening and anti-browning compound, CGA-lysine solutions

41 with and without cysteine at alkaline $\mathrm{pH}$ were compared. Given that cysteinyl thiol and $\varepsilon$ -

42 lysyl amine are two nucleophilic amino acid side groups competing for electrophilic o-

43 quinones at alkaline $\mathrm{pH}$, the mechanism of how cysteine inhibits the greening reaction

44 between lysine and CGA o-quinones at alkaline $\mathrm{pH}$ was elucidated. In the present study,

45 alkaline CGA-lysine solutions were prepared with different concentrations of cysteine to

46 simultaneously investigate cysteine’s role in non-enzymatic greening and browning by 
47 oxidized CGA reacting with amino acids in the presence of cysteine.

48 2. Materials and Methods Experimental design and preparation of CGA: lysine: cysteine solutions

50 L-cysteine effects on greening and browning were investigated in phosphate buffer $\mathrm{pH}$

518.0 and 9.0 CGA: lysine: cysteine solutions. Anhydrous L-cysteine hydrochloride of 98\%

52 purity, purchased from Acros Organics (New Jersey, USA), chlorogenic acid (>95\%;

53 TLC) and L-lysine (>98\%; TLC) purchased from Sigma-Aldrich (St. Louis, Missouri,

54 USA), were dissolved in $\mathrm{pH}$ 7.75, 8.0 and 9.0 phosphate buffers, and deionized water to

55 prepare L-cysteine, CGA, and L-lysine buffered stock solutions. Phosphate buffers were

56 prepared 24 hours in advance with $0.1 \mathrm{M}$ sodium phosphate solutions. Buffers were

57 adjusted to corresponding $\mathrm{pH}, 1.5$ hour before solutions were prepared. One hundred

58 milliliters of 5.6 mM CGA, 11.2 mM lysine solutions, and 10 mL 56 mM L-cysteine

59 solutions were prepared by dissolving $0.1984 \mathrm{~g}$ chlorogenic acid, $0.1637 \mathrm{~g}$ L-lysine and

$60 \quad 0.0883 \mathrm{~g} \mathrm{~L}$-cysteine $\cdot \mathrm{HCl}$ solids respectively into phosphate buffers. Buffered L-cysteine

61 solutions were diluted with phosphate buffer to achieve an L-cysteine concentration of 0 ,

62 1.02, 1.27, 2.55, 3.82 and $5.09 \mathrm{mM}$ in the final solutions. For each concentration of L-

63 cysteine, triplicates of each reaction mixture were prepared by mixing 1:1:0.2 (v:v:v)

64 stock solution of CGA: L-lysine :L-cysteine for a final concentration of $2.55 \mathrm{mM}$

65 CGA:5.09 mM Lys: 0-5.09 mM Cys. Two milliliters of buffered 5.6 mM CGA+ 2mL of

$66 \quad 11.2 \mathrm{mM}$ lysine solutions $+0.4 \mathrm{~mL}$ of $\mathrm{pH} 8.0$ or 9.0 phosphate buffer were prepared as

67 negative controls. As both amino acids are reactive with CGA, L-cysteine and L-lysine

68 solutions were mixed first, and reaction starting time points were recorded after adding

69 CGA into the mixture. 


\section{2 pH and UV-Vis scans of CGA: lysine: cysteine solutions}

71 To investigate the effect of $\mathrm{pH}$ on chlorogenic acid-amino acid non-enzymatic

72 browning and greening, buffered and unbuffered CGA: lysine: cysteine solutions were

73 monitored at $400 \mathrm{~nm}$ to $750 \mathrm{~nm}$ in a flat bottom Falcon 96 microplate with FLUOstar

74 Omega Microplate Reader (BMG Labtech, Ortenberg, Germany) over 48 hours of

75 incubation at ambient temperature.

$76 \mathrm{pH}$ readings were measured at each time point using a Vernier $\mathrm{pH}$ sensor (Vernier

77 Software and Technology, Beaverton, OR) after UV-Vis scans were taken.

$78 \quad 2.3$ Folin-Ciocalteu Reagent reducing and Trolox equivalent antioxidant capacity of

79 CGA: lysine: cysteine solutions

80 Folin-Ciocalteu Reagent reducing capacity of CGA: Lys: Cys solutions was

81 performed as outlined by Yu, Nanguet and Beta (2013). Gallic acid was used to construct

82 a standard curve. Reducing capacity of reaction solutions were recorded as mg gallic acid

83 equivalents per milliliter of solution.

84 Trolox equivalent antioxidant capacity assay conducted by measuring ABTS+ radical

85 scavenging activity was modified from Hwang, Zou, Wang and Lim (2018). The stock

86 radical solution was prepared by mixing $2.45 \mathrm{mM}$ potassium persulfate with $7 \mathrm{mM}$

$87 \mathrm{ABTS}+$ in the dark for $24 \mathrm{~h}$ at ambient temperature $\left(\sim 22^{\circ} \mathrm{C}\right)$. Twenty microliters of CGA:

88 Lys: Cys solutions and $180 \mu \mathrm{L} \mathrm{ABTS}+$ solution was reacted in 96-well Falcon plate.

89 Absorbance at $734 \mathrm{~nm}$ was taken after 10 mins on a microplate reader (FLUOstar Omega

90 Microplate Reader, BMG Labtech, Ortenberg, Germany), with results recorded as

91 millimoles of Trolox per milliliter of solution.

$92 \quad$ 2.4 HPLC quantification of unreacted chlorogenic acid and cysteinyl conjugates in 
94 Unreacted CGA and cysteinyl-CGA conjugates quantifications of 20\% diluted CGA:

95 Lys: Cys solutions with HPLC water were carried out on an Agilent 1100 series HPLC

96 (Santa Clara, CA, USA) with G1315B diode array detector monitoring at $294 \mathrm{~nm}, 320$

$97 \mathrm{~nm}, 450 \mathrm{~nm}$, and $680 \mathrm{~nm}$. A Phenomenex ${ }^{\circledR}$ Luna Omega $5 \mu \mathrm{C} 18$ (2) $100 \AA$ (150 × $2 \mathrm{~mm}$,

$98 \quad 1.5 \mu \mathrm{m}$ particle size) column was used with HPLC water acidified with $0.1 \%$ (v/v) formic

99 acid and acetonitrile acidified with $0.1 \%(\mathrm{v} / \mathrm{v})$ formic acid were as mobile phase A and B.

100 The gradient used was 0 min, $0 \% \mathrm{~B}$; $1.5 \mathrm{~min}, 22.0 \% \mathrm{~B} ; 4.5 \mathrm{~min}, 26.0 \% \mathrm{~B} ; 6 \mathrm{~min}, 28.0 \%$

$101 \mathrm{~B}$; 7-7.1 min, isocratic on $30 \% \mathrm{~B}$; 7.1-8 min, linear gradient from $30 \%$ to $100 \% \mathrm{~B}$; 8.0-10

$102 \mathrm{~min}$, isocratic on $100 \%$; $10-11 \mathrm{~min}$, isocratic on $0 \% \mathrm{~B}$ at $1.0 \mathrm{~mL} / \mathrm{min}$ flow rate with the

103 oven temperature controlled at $30^{\circ} \mathrm{C}$.

$104 \quad 2.5$ LC-MS analysis for chlorogenic acid-amino acid adducts

105 Chlorogenic acid, lysine and cysteine standards prepared in pH 8 and 9 buffers were

106 diluted 1:10 for LC-MS analysis. Phenolic-amino acid adducts were determined with

107 Dionex UltiMate3000 UHPLC equipped with an autosampler, and a Bruker Impact IITM

108 UHR-QTOF MS (Bruker, Billerica, MA, USA) was used. Temperature was set at $30{ }^{\circ} \mathrm{C}$,

109 and a flow rate was set at $0.3 \mathrm{~mL} / \mathrm{min}$. Mobile phase A was $0.1 \%$ formic acid in HPLC-

110 grade water. Gradient used was 0 min, 0\% B; 1-1.5 mins, linear gradient from 0\% to $15 \%$

111 B; 1.5 - 4.5 mins, linear gradient from 15\% to 30\%; 4.5 to 6.0 mins 30\% to 45\% B; 6 - 8

112 mins, linear gradient $45 \%$ to $100 \%$ B; 8 - 9 mins isocratic on $100 \%$ and $9-10$ mins $0 \%$ of

113 mobile phase B (0.1\% formic acid in HPLC-grade acetonitrile).

114 Mass spectrometry was carried out in the positive mode on a HP 1100MSD series

115 (Agilent Technologies) equipped with an ESI interface and LC/ MSD ChemStation 
116 software (Agilent Technologies, 1990 2002). The MS detector was in API-ES, positive

117 polarity mode, drying gas flow was $4.0 \mathrm{~L} / \mathrm{min}$ at $180^{\circ} \mathrm{C}$, nebulizer pressure was $35 \mathrm{psi}$,

118 capillary voltage was $4500 \mathrm{~V}$, and the mass spectra of eluate positive ions ranged from

$119 \mathrm{~m} / \mathrm{z} 50$ to 1200.

\section{$120 \quad 2.6$ Statistical Analysis}

121 A complete $2 \times 6 \times 3$ factorial design was implemented with $2 \mathrm{pH}$ (8 and 9), 6

122 cysteine concentrations ( $(0,1.02,1.27,2.55,3.82$ and $5.09 \mathrm{mM})$ and 10 different

123 incubation time points $(1-8,24$, and 48 h). The effect of cysteine concentration, $\mathrm{pH}$,

124 incubation time and their interactions were evaluated in a three-way factorial Analysis of

125 Variance (ANOVA): two fixed effects (cysteine concentration and $\mathrm{pH}$ ) as variables were

126 repeated at 10 different incubation time points. Duncan's Multiple Range test was used to

127 determine the significance level between browning/ $A_{410 n m}$, greening $/ A_{680 n m}$, and

128 antioxidant capacity) and three-way interactions of independent variables ( $\mathrm{pH}$ and

129 cysteine concentrations). Linear regression analysis was conducted to model the

130 relationship between color intensities $\left(\mathrm{A}_{410 \mathrm{~nm}}\right.$ and $\left.\mathrm{A}_{680 \mathrm{~nm}}\right)$ and cysteine concentration (0

131 to $5.091 \mathrm{mM}$ ) at 2 different $\mathrm{pH}(\mathrm{pH} 8$ and 9) and 10 different incubation time points.

132 Statistical Analysis Software 9.3 was used with the level of significance at $\alpha=0.05$ (SAS

133 Institute Inc., 2014).

\section{3. Results and Discussion}

135 3.1. Effects of cysteine concentration on color intensities

136 Colorless chemical compounds with carbonyl and primary amine groups are

137 precursors of brown pigments. Initial browning $\left(\mathrm{A}_{410 \mathrm{~nm}}\right)$ in CGA: Lys: Cys solutions was

138 primarily because CGA was oxidized to brown CGA quinones in alkaline pH solutions. 
139 Hypochromic shifts at $410 \mathrm{~nm}$ over $48 \mathrm{~h}$ incubation were due to consumption of brown

140 CGA quinones. Besides forming green pigments with amines, CGA quinones can also be

141 consumed by cysteine to form colorless cysteinyl-CGA conjugates, or cysteine could

142 reduce CGA quinones to colorless CGA diphenols, leading to lowered browning. From

14324 to $48 \mathrm{~h}$, hyperchromic shift at $410 \mathrm{~nm}$ was observed in all treatments except controls

144 at $\mathrm{pH} 8$ and 9 because regenerated CGA diphenols were oxidized again to brown CGA

145 quinones. After $48 \mathrm{~h}$, browning increased as cysteine concentration increased, which

146 might be due to higher amino acid concentration present in high cysteine solutions (Fig

$1471 \mathrm{~A} \& \mathrm{~B})$. Amines can react with quinones to form imines, which can then subsequently

148 undergo Maillard reaction to form melanoidins (Lund et al., 2011; Lund \& Ray, 2017).

149 Both cysteine and $\mathrm{pH}$ affected browning (Fig 1A\&B, Table S2).

150 No induction period was observed for green color development (Fig 1C\&D) as

151 primary amines are prone to nucleophilically attack CGA quinones under alkaline

152 conditions (Rawel \& Rohn, 2010). Increased greening and decreased browning were

153 observed after $24 \mathrm{~h}$, attributed to consumption of brown CGA quinones to form green

154 pigments over time. Given that free amine groups of lysine primarily react with CGA

155 quinones to form $\mathrm{N}$-quinonyl conjugates which would further oxidatively cyclize to green

156 pigments, lysyl amines became less available to form imines, which are precursors of

157 melanoidins (Lund \& Ray, 2017), as high abundance of lysyl amine-CGA conjugates but

158 not iminoquinones were identified as predominant reaction products in alkaline CGA:

159 Lys: Cys solutions (Table 1).

160 In CGA: Lys: 0-5.09 mM Cys solutions, a hypochromic shift at $680 \mathrm{~nm}$ was

161 observed as cysteine increased at both $\mathrm{pH} 8$ and 9 (Fig 1C\&D). Stronger negative 
162 correlations between cysteine concentration and greening $\left(\mathrm{A}_{680 \mathrm{~nm}}\right)$ were observed as

163 solutions were incubated over time. Cysteine inhibited CGA quinone-lysine greening in a

164 concentration dependent manner. After $48 \mathrm{~h}$, greening occurred at lower cysteine

165 concentration while no visible greening was observed when cysteine was $5.09 \mathrm{mM}$ at

166 both pH 8 and 9 (Fig 1C\&D). Since highest cysteine concentration of $5.09 \mathrm{mM}$ was at the

167 same concentration as lysine in non-green CGA: Lys: Cys solutions, cysteinyl thiols (-

$168 \mathrm{SH}$ ) had a higher affinity than $\varepsilon$-lysyl amine for CGA o-quinones for nucleophilic

169 conjugation, similar to findings by Lund and Ray (2017) and Pierpoint (1966) who found

170 that cysteinyl thiols have a higher affinity to CGA o-quinones than $\alpha$-amines from amino

171 acids. When cysteine concentrations were less than that of lysine, lack of greening was

172 temporary as CGA quinones that were reduced to CGA hydroquinones could result in

173 regenerated CGA hydroquinones undergoing further oxidative reactions to form green

174 TBA or yellow TBA derivatives with time. The 830 Da yellow TBA derivatives (Fig 2)

175 were not oxidized to green color in the first $24 \mathrm{~h}$.

176 As cysteine concentration increased, CGA quinones-lysine induced greening in

177 buffered pH 8 and 9 CGA: Lys: mM Cys solutions decreased by forming colorless

178 cysteinyl-CGA conjugates (Fig 2), and by reducing quinones to CGA di-phenols (Fig 3)

179 to limit availability of CGA quinones, one of the precursors of green trihydroxy

180 benzacridine. At pH 8 and 9, CGA quinones were first attacked at the most electrophilic

181 position 2 of the phenol ring by nucleophiles, cysteinyl thiols or $\varepsilon$-lysyl amines, to form

182 cysteinyl-CGA conjugates (compound 1, 3\&4, Table 1) or lysyl amine-CGA conjugates

183 (compound 2, Table 1). Formation of di-cysteinyl CGA conjugates (compound 4, Table

184 1) was reported by Pierpoint (1966) and Schilling et al. (2008) which was substituted at 
185 position 5 of the phenol ring when excess thiols were present because the $5^{\text {th }}$ position was

186 the second most electrophilic position on a CGA o-quinone. When CGA reacted with

187 lysine, the $2^{\text {nd }}$ position of CGA quinone could also be nucleophilically attacked by lysyl

188 amines, leading to formation of green TBA derivatives (Fig 2).

189 Given the reducing capacity of thiols, cysteine was able to reduce brown CGA

190 quinone monomers to CGA hydroquinone dimers (Fig 3, compound 7 in Table 1). Once

191 regenerated CGA hydroquinone dimers were oxidized to quinones. Dimer CGA quinones

192 can be attacked by amines to form green TBA derivatives or substituted with cysteinyl

193 thiols, consistent with Cao and Xiong (2015) who reported nucleophilic amine and thiol

194 conjugation of CGA quinone monomers and dimers.

\section{3.2. Effects of $\mathbf{p H}$ on color intensities}

196 In the first experiment, when cysteine was increased from 0 to $1.486 \mathrm{mM}$, greening at

$197 \mathrm{pH} 9$ was strongly negatively correlated with cysteine concentration $(\mathrm{r}=-0.79817$ to -

198 0.97579, $\mathrm{p}<0.0001)$ throughout incubation (Fig S2). Moderate and weak correlations

199 between cysteine concentration and greening were observed at $\mathrm{pH} 8$ and $7.75\left(\mathrm{r}_{\mathrm{pH}}=-\right.$

200 0.70406, $\left.\mathrm{p}=0.0034 ; \mathrm{r}_{\mathrm{pH} 7.75}=-0.50525, \mathrm{p}=0.0547\right)$. Stronger correlations between

201 cysteine concentration and greening were observed at pH 9. pH 9 was above both CGA

202 phenolic pKa 8.42 and cysteine thiol pKa 8.3 resulting in high abundance of thiolates in

$203 \mathrm{pH} 9$ solutions. This greater anti-greening effect by cysteine with higher $\mathrm{pH}$ was also

204 observed when cysteine was increased to $5.09 \mathrm{mM}$ in buffered pH 8 and 9 CGA: Lys:

205 Cys solutions (Fig 1C\&D).

206 The phenolic pKa of CGA of 8.42 is lower than that in $\mathrm{pH} 9$ solutions resulting in

207 higher initial browning in $\mathrm{pH} 9$ solutions than in $\mathrm{pH} 8$ solutions (Fig A\&B). At alkaline

$208 \mathrm{pH}$, only CGA phenolates were oxidized to brown CGA o-quinone monomers, so less 
209 CGA were prone to oxidation at lower $\mathrm{pH}$. As phenolic ions gave rise to CGA o-quinone

210 formation (Cilliers \& Singleton, 1989), $\mathrm{A}_{410 \mathrm{~nm}}$ in $\mathrm{pH} 9$ buffered solutions was twice as

211 high as in $\mathrm{pH} 8.0$ buffered solutions ( $<<0.0001$, Fig 1A\&B).

212 Highly electrophilic CGA o-quinones formed by non-enzymatic oxidation could be

213 stabilized by both thiol and amine nucleophilic addition (Penalver et al., 2002). When

214 lysyl amines nucleophilically attacked CGA quinones, formation of green TBA

215 derivatives lead to hyperchromic shift at $680 \mathrm{~nm}$ after $48 \mathrm{~h}$ in buffered CGA: Lys: Cys

216 solutions at all tested pHs and cysteine concentrations (Fig 1C\&D). Greening in pH 9.0

217 CGA: lysine: cysteine solutions was significantly higher than that in $\mathrm{pH} 8.0$ buffered

218 solutions $(\mathrm{p}<0.0001)$ at each tested cysteine concentration. Higher greening in $\mathrm{pH} 9$

219 buffered solutions was attributed to deprotonation of both CGA (pKa of phenol: 8.42)

220 and lysine (pKa of $\alpha$-amine: 8.95). At $\mathrm{pH}$ 9, besides facilitating CGA o-quinones

221 formation, deprotonation of $\alpha$-lysyl amine group (pKa 8.90) made $\varepsilon$-lysyl amine group

222 (pKa 10.28), the primary site to covalently bond with CGA o-quinones to form amine-

223 quinone adducts, followed by Diels-Alder cyclization and dehydration to yellow

224 pigmented TBA derivatives (Fig 2), which became green once oxidized (Yabuta et al.,

225 2001). In pH 8.0 buffered solutions, lower greening after 48 hours incubation occurred

226 because less $\mathrm{o}$-quinones were available at $\mathrm{pH} 8$ compared to $\mathrm{pH} 9$ to form green TBA

227 derivatives and CGA o-quinones had a lower reactivity in $\mathrm{pH} 8$ conditions (Bongartz et

228 al., 2016; Murata et al., 2002). At the same cysteine concentration, pH 9 CGA: Lys: Cys

229 solutions had significantly higher greening because higher $\mathrm{pH}$ promoted oxidative

230 reactions including formation CGA-quinones and TBA. More thiyl radicals would form

231 at higher $\mathrm{pH}$, facilitating oxidation to green TBA derivatives. In addition, more thiyl 
232 radicals gave rise to cysteinyl-CGA conjugates. Thus, greater inhibition of greening was

233 observed at $\mathrm{pH} 9$ than 8 , with a $94 \%$ decrease in greening from 0 to $5.09 \mathrm{mM}$ cysteine at

$234 \mathrm{pH} 9$ compared to 87\% decrease at pH 8 (Fig 1C\&D), and lowering pH had a greater

235 anti-greening effect than cysteine concentration (Table S2).

236 3.3. Formation of conjugates over $48 \mathrm{~h}$ incubation

237 At alkaline pH, electrophilic CGA-quinones (353 Da, Fig 2) were subject to

238 nucleophilic attack by amines or thiols in buffered CGA: Lys: Cys solutions. When the

239 highly reactive CGA-quinones (353 Da) were stabilized by amine addition, amine-

240 hydroquinone adducts (499 Da) were formed, which were subsequently cyclized with

241 another CGA-quinone by Diels-Alder reaction and oxidative elimination to form

242 trihydroxy benzacridine (TBA) structures (830 Da). The hydroxy groups oxidized into

243 ketones at alkaline $\mathrm{pH}(829,828 \mathrm{Da}$, Fig 2), and thus greening was observed in $\mathrm{pH} 8$ and

2449 CGA: Lys: Cys solutions except with $5.09 \mathrm{mM}$ cysteine. Formation of TBA derivatives

245 led to a hyperchromic shift at $680 \mathrm{~nm}$ in pH 8 and 9 buffered CGA: Lys: Cys solutions

246 (Fig 1C\&D), implying a simultaneous consumption of brown CGA quinones

247 (correlations for browning and cysteine concentration: $\mathrm{r}_{\mathrm{pH} 8}=-0.5707$ to $0.9149 ; \mathrm{r}_{\mathrm{pH9}}=-$

$248 \quad 0.7130$ to 0.9551$)$.

249 Formation of green TBA derivatives was inhibited by increased cysteine

250 concentration and lowered pH (Fig 1 C\&D), consistent with HPLC data shown in Fig S1.

251 Two absorbance peaks were observed at $320 \mathrm{~nm}$ (Fig S1 A\&B): one at 1.60 minutes, the

252 other eluted at 1.75 minutes. The three-way interactions between cysteine concentration $\mathrm{x}$

$253 \mathrm{pH}$ x incubation time were significant for compounds eluting at 1.60 and 1.75 minutes

254 (Table S1; Table S2). The compounds eluting at 1.60 minutes were detected at all $3 \lambda \mathrm{s}$ :

255320,450 and $680 \mathrm{~nm}$, and were thus identified as green TBA derivatives. After 48h, the 
256 relative abundance of TBA derivatives quantified by HPLC absorbance peak area

257 decreased from 1870.00 to 1539.28 and from 3891.87 to 2657.22 , as cysteine increased at

258 pH 8 and 9, respectively (Table S1), which was consistent with hypochromic shift at 680

$259 \mathrm{~nm}$ at $\mathrm{pH} 8$ and 9 (Fig 1 C\&D).

260 Signals of the compounds that eluted at 1.75 minutes were only detected at $320 \mathrm{~nm}$

261 but not $450 \mathrm{~nm}$ and $680 \mathrm{~nm}$, indicating a hydroxycinnamic acid (HCA) based phenolic

262 structure (C3-C6 phenolics). Since CGA and caffeic acid standards eluted at 2.40 and

2632.71 minutes respectively, the compounds that eluted at 1.75 minutes were possibly CGA

264 conjugates, whose relative abundance increased from 6300.74 to 7971.60 and from

2652284.2 to 5647.92, as cysteine increased at pH 8 and 9, respectively. The 1.75 min eluted

266 compounds were tentatively identified as coeluted lysyl amine-CGA and cysteinyl-CGA

267 conjugates because the peak height decreased while the peak bottom became wider (peak

268 tailing) as cysteine concentration increased and the relative abundance in controls were

269 not zero at both $\mathrm{pH}$. In addition, the relative abundance of this compound(s) decreased

270 throughout incubation (Table S1). It was thus concluded that CGA quinones react with

271 lysyl amines to form amine-CGA conjugates before cyclization with another CGA

272 quinone to form green TBA derivatives (Fig 2).

273 Lysyl amine-CGA conjugates and green TBA derivatives were identified in all CGA:

274 Lys: Cys solutions at pH 8 and 9. Compound 2 (Table 1) was identified as a lysine-CGA

275 conjugate with a sodium ion, as all CGA: Lys: Cys solutions were prepared in sodium

276 phosphate buffer. The predominant fragment $[\mathrm{M}+\mathrm{H}]+$ of compound 1 was at $\mathrm{m} / \mathrm{z}$ 147,

277 consistent with a lysine moiety in the structure, which can be further fragmented to $\mathrm{m} / \mathrm{z}$

278130 as $\mathrm{C}_{6} \mathrm{H}_{12} \mathrm{NO}_{2}$ (2-Aminohexanoate isomer), and $\mathrm{m} / \mathrm{z} 84$ as $\mathrm{C}_{4} \mathrm{H}_{6} \mathrm{NO}$ (1-Oxo-2-buten- 
279 2-yl-amino). Ion $\mathrm{m} / \mathrm{z} 311$ was 164 Da higher than fragment at m/z 147, indicating a

$280 \mathrm{C}_{9} \mathrm{H}_{8} \mathrm{O}_{3}$ ion (caffeic aldehyde isomer) was attached. The precursor ion $\mathrm{m} / \mathrm{z} 527$ was 23

281 Da higher than predicted lysine-CGA conjugate at $\mathrm{m} / \mathrm{z}$ 504, suggesting a sodium ion was

282 attached to lysine-CGA conjugates (Fig 4B). Higher signal intensity (89433 at pH 8;

28379336 at $\mathrm{pH}$ 9) of compound 2 in $\mathrm{pH} 8$ than $\mathrm{pH} 9$ solutions at the same cysteine

284 concentrations, indicated that lysyl amine-CGA conjugate was a precursor of green TBA

285 in alkaline CGA: Lys: Cys solutions, as more of it was consumed in pH 9 than 8

286 solutions. Lysine-CGA iminoquinone ( $\mathrm{m} / \mathrm{z}$ 481, Fig 2) was postulated as an intermediate

287 of green TBA formation (Yabuta et al., 2001), which was not a predominant reaction

288 product identified in this study. Given the high abundance of lysyl amine-CGA

289 conjugates (Fig 4B), lysine reacting with CGA quinones preferentially to form lysyl

290 amine-CGA conjugates, which was 10 times more than cysteinyl-CGA conjugates,

291 followed by a dehydro-Diels-Alder reaction was the primary route to form green TBA

292 (Fig 2).

293 Compound 5 (Table 1, Fig 4E) was identified as trihydroxy benzacridine (TBA, m/z

294 830) with a loss of a carboxy group (44 Da) on a quinic acid moiety (174 Da). Ion m/z

295263 was a trihydroxy benzacridine core structure, $\mathrm{C}_{16} \mathrm{H}_{8} \mathrm{NO}_{3}$ (6-Oxo-2,6-dihydronaphtho-

296 indole-1-carboxylate), consistent with TBA fragments reported by Bongartz et al. (2016)

297 and Schilling et al (2008). Ion m/z 525 was a trihydroxy benzacridine core structure,

$298 \mathrm{C}_{16} \mathrm{H}_{8} \mathrm{NO}_{3}$, attached with a quinic acid (191 Da), a carboxy group (44 Da) and a carbonyl

299 group (28 Da), when a quinic acid moiety (174 Da) and a lysine moiety (129 Da) were

300 fragmented from a parent TBA molecule ( $\mathrm{m} / \mathrm{z}$ 830). Higher signal intensity of compound 
3015 in $\mathrm{pH} 9$ than $\mathrm{pH} 8$ solutions was consistent with TBA quantification by HPLC (Table

302 S1) that more green TBA derivatives were formed at higher $\mathrm{pH}$.

303 Cysteine reacted with CGA-quinones in alkaline CGA: Lys: Cys solutions primarily

304 by redox phenol regeneration with formation of cystine (240 Da) and CGA dimers (707

305 Da, Fig3). Compound 6 and 7 were in high abundance and were identified as cystine-

306 cystine crosslinks (m/z 481, Fig4F) and CGA dimers (m/z 708) respectively. Compound

3077 (m/z 731) was 23 Da higher than a CGA dimer (m/z 708, Fig 4G), identified as a CGA

308 dimer with a sodium ion, whose abundance was $60 \%$ higher in $\mathrm{pH} 8$ than $\mathrm{pH} 9$ solutions.

309 Cysteine was more prone to regenerate CGA diphenols at $\mathrm{pH} 8$ when $\mathrm{pH}$ was below its

310 thiol pKa 8.3.

311 At pH 9, more cysteine reacted with CGA o-quinones by thiol-conjugation: thiyl

312 radical nucleophilically added to yellow electrophilic $o$-quinones to form colorless thiol-

313 ether conjugates, cysteinyl-CGA conjugates, which were subject to secondary thiol

314 substitution to form di-cysteinyl CGA conjugates (Cilliers \& Singleton, 1990; Schilling et

315 al., 2008). Both mono-cysteinyl-CGA (compound 1), and di-cysteinyl-CGA (compound

316 4) conjugates were identified in alkaline CGA: Lys: Cys solutions. Compound 1 (Table

317 1, Fig A) was identified as a mono-cysteinyl-CGA conjugate (m/z 475) lost a carboxyl

318 group (44 Da). The predominant fragment $[\mathrm{M}+\mathrm{H}]^{+}$of compound 1 was at $\mathrm{m} / \mathrm{z} 227$,

319 identified as a thiol-methyl caffeate conjugate, $\mathrm{C}_{10} \mathrm{H}_{10} \mathrm{O}_{4} \mathrm{~S}$. Ion m/z 431 was $164 \mathrm{Da}$

320 higher than fragment at $\mathrm{m} / \mathrm{z} 147$, indicating a $\mathrm{C}_{9} \mathrm{H}_{8} \mathrm{O}_{3}$ ion (caffeic aldehyde isomer) was

321 attached. As pH decreased from 9 to 8, 50\% decreased signal intensity of compound 1

322 was observed when cysteine concentration was kept constant. Compound 3 (Table 1),

323 which fragmented in a similar pattern as compound 1 did was identified as a 2 mono- 
324 cysteinyl-CGA conjugate (m/z 475) crosslinked together (m/z 947). Compound 4 (Table

325 1, Fig 4D) was identified as di-cysteinyl-CGA conjugates (m/z 595) plus a carboxy group

326 with a sodium ion. The predominant fragment $[\mathrm{M}+\mathrm{H}]^{+}$was the same as that of compound

3271 and 3, a thiol-methyl caffeate conjugate, $\mathrm{C}_{10} \mathrm{H}_{10} \mathrm{O}_{4} \mathrm{~S}$, at $\mathrm{m} / \mathrm{z}$ 227. Precursor ion m/z 663

328 was 68 Da higher than di-cysteinyl-CGA conjugates at $\mathrm{m} / \mathrm{z} 595$, which could be a

329 protonated carboxy group (45 Da) and a sodium ion (23 Da). More di-cysteinyl CGA

330 conjugates were formed in $\mathrm{pH} 9$ than $\mathrm{pH} 8$ CGA: Lys: Cys solutions. Compared with $\mathrm{pH}$

3319.0 solutions, $\mathrm{pH} 8.0$ solutions were below cysteinyl thiol $\mathrm{pKa}(\sim 8.3)$. The ratio of thiyl

332 radical (-S-) to sulfhydryl (-SH) was higher in $\mathrm{pH} 9.0$ than 8.0 buffered solutions, so

333 cysteinyl thiols were more prone to form colorless cysteinyl-CGA conjugates at $\mathrm{pH} 9$

334 while more reductively regenerated CGA dimers were found in CGA: Lys: Cys solutions.

335 However, brown solutions were observed in CGA: Lys: 5.09 mM Cys solutions (Fig

336 1C\&D). As imine formation was retarded with presence of cysteine (Zeng et al., 2017;

337 Zhang et al., 2018), hypochromic shift at $410 \mathrm{~nm}$ in non-green solution might be induced

338 by oxidation cysteinyl-CGA conjugates.

339 Overall, at the same alkaline $\mathrm{pH}$, the abundance of mono-cysteinyl CGA

340 conjugates increased while the formation of lysyl amine-CGA conjugates remained

341 unchanged when cysteine concentration was increased from 0 to $5.09 \mathrm{mM}$. When

342 cysteine and lysine were both at $5.09 \mathrm{mM}$, no visible greening was observed at either $\mathrm{pH}$

3438 or $\mathrm{pH}$ 9. Simultaneously, the amount of CGA dimers in $\mathrm{pH} 8$ was $60 \%$ higher than in

$344 \mathrm{pH} 9$ solutions, indicating cysteine was primarily reducing brown CGA $o$-quinones to

345 CGA hydroquinone dimers when $\mathrm{pH}$ was below cysteinyl thiol pKa 8.3. 


\subsection{Individual and combined effects of cysteine concentration and $\mathrm{pH}$ on}

348 Cysteinyl thiol and di-thiol CGA conjugates were expected to exhibit antioxidant

349 capacity, given the similarity of caffeic acid and CGA, an ester of caffeic acid. Fujimoto

350 et al. (2013) reported mono-thiol methyl caffeate adduct scavenged ethyl linoleate

351 hydroperoxide radicals nearly twice as much as methyl caffeate did. Miura et al. (2014)

352 found cysteinyl caffeic acids successfully maintained desirable bright red color of meat

353 by reducing metmyoglobin to oxymyoglobin.

354 Antioxidant capacity by Trolox equivalent antioxidant (TEAC) assay was primarily

355 measuring the potential to neutralize free radicals, as TEAC measures the ionization

356 potential equivalent. Antioxidant capacity by TEAC at pH 8 was significantly higher than

357 pH 9 (Fig 5A), because lysine-CGA interaction was higher at pH 9 than $\mathrm{pH} 8$ and thus

358 more CGA was consumed to produce green TBA. As cysteine concentration increased,

359 TEAC at both pH 8 and 9 increased as more mono-cysteinyl-CGA conjugates (Fig

360 4A\&C) were formed. Plateaued TEAC was observed at both tested pH when cysteine

361 was higher than $1.27 \mathrm{mM}$, consistent with Fujimoto et al. (2013) who found that

362 additional cysteinyl thiol would not cause further antioxidant capacity enhancement, as

363 excess cysteine might be consumed by secondary thiol substitution (Fig 4D).

364 Antioxidant capacity by Folin-Ciocalteu reagent reducing capacity (FCRC) assay to

365 assess electron transferring ability, showed increasing FCRC at both pH 8 and 9 as

366 cysteine concentration increased. High FCRC in pH 8 control might be attributed to the

367 reducing capacity of free CGA (Fig 4B), which after 48 h (Fig 5C) was still available

368 because less thiyl radicals and deprotonated $\varepsilon$-lysyl amines were available to form CGA

369 conjugates at $\mathrm{pH} 8$. 
371 higher TEAC because cysteine protected more CGA at pH 8 by reducing CGA quinones

372 to CGA hydroquinone dimers, which had 60\% higher signal intensity in $\mathrm{pH} 8$ than $\mathrm{pH} 9$

373 solutions (Fig 4G). As cysteine concentration increased from $1.02 \mathrm{mM}$ to $5.09 \mathrm{mM}, 20 \%$

374 and 39\% higher FCRC were found when $\mathrm{pH}$ remained the same at 8 and 9 respectively.

375 When cysteine concentration was kept constant, reducing capacity was increased when

$376 \mathrm{pH}$ was lowered from 9 to 8 , indicating antioxidant capacity could be enhanced by

377 lowering $\mathrm{pH}$. Compared to controls with no added cysteine, FCRC at $\mathrm{pH} 8$ was nearly

$37860 \%$ higher than that at $\mathrm{pH} 9$. With addition of cysteine, the FCRC increased to a greater

379 extent (21.5\%) at $1.02 \mathrm{mM}$ cysteine while it only increased by $4.7 \%$ at $5.09 \mathrm{mM}$ cysteine

380 when $\mathrm{pH}$ was lowered from 9 to 8 , suggesting the enhanced reducing capacity might be

381 due to formation of cysteinyl-CGA conjugates instead of increased cysteine in the

382 solutions. Although addition of cysteine and lowering $\mathrm{pH}$ both enhanced antioxidant

383 capacity $(\mathrm{p}<0.0001$, Table S2), cysteine exhibited a greater effect. The individual and

384 combined effects of cysteine concentration and $\mathrm{pH}$ enhanced more on reducing than

385 radical scavenger capacity (Table S2).

\section{4. Conclusions}

387 Concentration dependent cysteine competitive inhibition of CGA-lysine induced 388 greening was observed when cysteine was added from 0 to $5.09 \mathrm{mM}$. Adding cysteine to

389 CGA-lysine solutions promotes formation of mono- and di-cysteine-CGA conjugates,

390 which also contribute to a high antioxidant capacity. When $\mathrm{pH}$ was above 8.3, cysteine

391 lowered alkaline CGA-lysine greening by preferentially reacting with CGA quinones

392 because cysteinyl thiols have a higher affinity for CGA quinones than $\varepsilon$-lysyl amine. 
Cysteinyl thiol's reducing effect on CGA quinones resulted in phenol regeneration and

394 formation of CGA dimers when $\mathrm{pH}$ was below 8.3. Higher cysteine concentration

395 enhanced antioxidant capacity with an anti-greening effect.

396 In conclusion, adding cysteine in alkaline $\mathrm{pH}$ environments could be a potential

397 anti-greening approach. Sunflower protein when alkaline extracted could green due to its

398 high CGA content. The formation of cysteinyl CGA could exhibit a protective effect

399 towards oxidation of extracted protein.

400

401 Conflict of Interest

402 Authors declare no conflict of interest.

403 Acknowledgements

404 This research did not receive any specific grant from funding agencies in the public,

405 commercial, or not-for-profit sectors. Authors would like to thank Chapman University

406 for funding this research.

\section{References}

408

409

410

411

412

413

414

415

416

417

418

419

420

421

422

423

Altunkaya, A., \& Gökmen, V. (2009). Effect of various anti-browning agents on phenolic compounds profile of fresh lettuce (L. sativa). Food Chemistry, 117, 122-126. doi:10.1016/j.foodchem.2009.03.085

Ashoor, S. H., \& Zent, J. B. (1984). Maillard browning of common amino acids and sugars. Journal of Food Science (USA)(4), 1206-1207.

Atonfack, J. T., Ataman, Z. A., \& Were, L. M. (2019). Acidulant Effect on Greening, Reducing Capacity, and Tryptophan Fluorescence of Sunflower Butter Cookie Dough During Refrigerated Storage. Journal of the Science of Food and Agriculture, 99(5), 2186-2193.

Bassil, D., Makris, D. P., \& Kefalas, P. (2005). Oxidation of caffeic acid in the presence of Lcysteine: isolation of 2-S-cysteinylcaffeic acid and evaluation of its antioxidant properties. Food research international(4), 395-402.

Bittner, S. (2006). When quinones meet amino acids: chemical, physical and biological consequences. Amino Acids, 30(3), 205-224.

Bongartz, V., Brandt, L., Gehrmann, M. L., Zimmermann, B. F., Schulze-Kaysers, N., \& Schieber, A. (2016). Evidence for the Formation of Benzacridine Derivatives in AlkalineTreated Sunflower Meal and Model Solutions. Molecules, 21(1), 91-99. 
424

425

426

427

428

429

430

431

432

433

434

435

436

437

438

439

440

441

442

443

444

445

446

447

448

449

450

451

452

453

454

455

456

457

458

459

460

461

462

463

464

465

466

467

468

469

470

471

472

473

474

Budryn, G., \& Rachwal-Rosiak, D. (2013). Interactions of Hydroxycinnamic Acids with Proteins and Their Technological and Nutritional Implications. Food Reviews International, 29(3), 217-230. doi:10.1080/87559129.2012.751545

Cao, Y., \& Xiong, Y. L. (2015). Chlorogenic acid-mediated gel formation of oxidatively stressed myofibrillar protein. Food Chemistry, 180, 235-243. doi:10.1016/j.foodchem.2015.02.036

Chapin, M. M., Rochette, L. M., Annest, J. L., Haileyesus, T., Conner, K. A., \& Smith, G. A. (2013). Nonfatal choking on food among children 14 years or younger in the United States, 2001-2009. Pediatrics(2), 275-284.

Cilliers, J. J. L., \& Singleton, V. L. (1989). Nonenzymic autoxidative phenolic browning reactions in a caffeic acid model system. Journal of agricultural and food chemistry, 890896.

Cilliers, J. J. L., \& Singleton, V. L. (1990). Caffeic acid autoxidation and the effects of thiols. Journal of agricultural and food chemistry (USA)(9), 1789-1796

Cilliers, J. J. L., \& Singleton, V. L. (1991). Characterization of the products of nonenzymic autoxidative phenolic reactions in a caffeic acid model system. Journal of agricultural and food chemistry (USA)(7), 1298-1303.

Danilewicz, J. C., Tunbridge, P., \& Kilmartin, P. A. (2019). Wine Reduction Potentials: Are These Measured Values Really Reduction Potentials? Journal of agricultural and food chemistry(15), 4145-4153.

Eardley, M. (2018). Capitalize on the Growing Trend of Plant-Based Proteins. Winsight Grocery Business. Retrieved from https://www.winsightgrocerybusiness.com/retailfoodservice/capitalize-growing-trend-plant-based-proteins

Eric, K., Raymond, L. V., Abbas, S., Song, S., Zhang, Y., Masamba, K., \& Zhang, X. (2014). Temperature and cysteine addition effect on formation of sunflower hydrolysate Maillard reaction products and corresponding influence on sensory characteristics assessed by partial least square regression. Food Research International, 57, 242-258. doi:10.1016/j.foodres.2014.01.030

Eric, K., Raymond, L. V., Huang, M., Cheserek, M. J., Hayat, K., Savio, N. D., . . Zhang, X. (2013). Sensory attributes and antioxidant capacity of Maillard reaction products derived from xylose, cysteine and sunflower protein hydrolysate model system. Food Research International, 54, 1437-1447. doi:10.1016/j.foodres.2013.09.034

FDA. (2019). 2018 Recalls, Market Withdraws, and Safety Alerts. Retrieved from https://www.fda.gov/safety/archive-recalls-market-withdrawals-safety-alerts/2018recalls-market-withdrawals-safety-alerts

Ferrer, B. (Producer). (2019). Givaudan unveils "most promising new plant-based proteins" in collaboration with UC Berkeley. Retrieved from https://www.foodingredientsfirst.com/news/givaudan-unveils-most-promising-new-plantbased-proteins-in-collaboration-with-uc-berkeley.html

Fujimoto, A., Inai, M., \& Masuda, T. (2013). Chemical evidence for the synergistic effect of a cysteinyl thiol on the antioxidant activity of caffeic and dihydrocaffeic esters. Food Chemistry, 138, 1483-1492. doi:10.1016/j.foodchem.2012.11.073

Gabriel, A. A., Joe, A. V., \& Patrick, E. D. (2014). Folin-Ciocalteau Reagent for Polyphenolic Assay. International Journal of Food Science, Nutrition and Dietetics (IJFS)(8), 147156.

Gonzales-Perez, S., Merck, K. B., Vereijken, J. M., Koningsveld, v., G.A., \& Gruppen, H., Voragen, A.G.J. (2002). Isolation and characterization of undenatured chlorogenic acid free sunflower (Helianthus annuus) Proteins. Journal of Agricultural and Food Chemistry, 50, 1713-1719.

Hartman, G. (Producer). (2019). Saying No to Meat. What's Behind the Rising Interest in PlantBased Protein? Newsletter. 
475

476

477

478

479

480

481

482

483

484

485

486

487

488

489

490

491

492

493

494

495

496

497

498

499

500

501

502

503

504

505

506

507

508

509

510

511

512

513

514

515

516

517

518

519

520

521

522

523

524

525

Hayase, F., Kim, S. B., \& Kato, H. (1984). Decolorization and degradation products of the melanoidins by hydrogen peroxide. Agricultural and Biological Chemistry (Japan)(11), 2711-2717.

Hwang, S. H., Zuo, G., Wang, Z., \& Lim, S. S. (2018). Novel aldose reductase inhibitory and antioxidant chlorogenic acid derivatives obtained by heat treatment of chlorogenic acid and amino acids. Food Chemistry, 266, 449-457. doi:10.1016/j.foodchem.2018.06.053

Iacomino, M., Weber, F., Gleichenhagen, M., Pistorio, V., Panzella, L., Pizzo, E., . . . Napolitano, A. (2017). Stable Benzacridine Pigments by Oxidative Coupling of Chlorogenic Acid with Amino Acids and Proteins: Toward Natural Product-Based Green Food Coloring. 65, 6519-6528. doi:10.1021/acs.jafc.7b00999

Indrani, D., Prabhasankar, P., Rajiv, J., \& Rao, G. V. (2003). Scanning Electron Microscopy, Rheological Characteristics, and Bread-Baking Performance of Wheat-Flour Dough as Affected by Enzymes. Journal of Food Science(9), 2804-2812.

Jackson, H., \& Kendal, L. P. (1949). The Oxidation of Catechol and Homocatechol by Tyrosinase in the Presence of Amino-acids. Journal of Biochemistry, 44, 477-487.

Kalaras, M. D., Richie, J. P., Calcagnotto, A., \& Beelman, R. B. (2017). Mushrooms: A rich source of the antioxidants ergothioneine and glutathione. 233, 429-433. doi:10.1016/j.foodchem.2017.04.109

Kuijpers, T. F. M. (2013). Inhibition of tyrosinase-induced enzymatic browning by sulfite and natural alternatives. (PHD). Wageningen University, Wageningen University, Wageningen, NL. (978-94-6173-668-0)

Kuijpers, T. F. M., Narvaez Cuenca, C. E., Vincken, J. P., Verloop, J. W., Berkel, v., W.J.H., \& Gruppen, H. (2012). Inhibition of Enzymatic Browning of Chlorogenic Acid by SulfurContaining Compounds. 3507-3514.

Kumar, N., Khatkar, B. S., \& Kaushik, R. (2013). Effect of reducing agents on wheat gluten and quality characteristics of flour and cookies. Annals of the University Dunarea de Jos of Galati Fascicle VI -- Food Technology, 37(2), 68-81.

Lan, X., Liu, P., Xia, S., Jia, C., Mukunzi, D., Zhang, X., . . Xiao, Z. (2010). Temperature effect on the non-volatile compounds of Maillard reaction products derived from xylosesoybean peptide system: Further insights into thermal degradation and cross-linking. Food chemistry(4), 967-972. doi:10.1016/j.foodchem.2009.11.033

Lester, M. R. (1995). Sulfite sensitivity: significance in human health. Journal of the American College of Nutrition (USA)(3), 229-235.

Li, W.-W., Wolfgang, H., \& Jurgen, H. (2005). Site-specific binding of quinones to proteins through thiol addition and addition-elimination reactions. Journal of the American Chemical Society(17), 6140-6141.

Liang, S., Were, L., \& Hanh Lan, T. (2018). Lowering greening of cookies made from sunflower butter using acidic ingredients and effect on reducing capacity, tryptophan and protein oxidation. Food Chemistry, 252, 318-326.

Liang, S., \& Were, L. M. (2018a). Chlorogenic acid induced colored reactions and their effect on carbonyls, phenolic content, and antioxidant capacity in sunflower butter cookies. LWTFood Science and Technology, 87, 16-22. doi:10.1016/j.lwt.2017.08.069

Liang, S., \& Were, L. M. (2018b). Chlorogenic acid oxidation-induced greening of sunflower butter cookies as a function of different sweeteners and storage conditions. Food Chemistry, 241, 135-142.

Lund, M. N., Heinonen, M., Baron, C. P., \& Estevez, M. (2011). Protein oxidation in muscle foods: A review. Molecular Nutrition \& Food Research, 55, 83-95. doi:10.1002/mnfr.201000453

Lund, M. N., \& Ray, C. A. (2017). Control of Maillard Reactions in Foods: Strategies and Chemical Mechanisms. Journal of Agricultural and Food Chemistry, 65, 4537-4552. doi:10.1021/acs.jafc.7b00882 
526

527

528

529

530

531

532

533

534

535

536

537

538

539

540

541

542

543

544

545

546

547

548

549

550

551

552

553

554

555

556

557

558

559

560

561

562

563

564

565

566

567

568

569

570

571

572

573

574

575

576

Maegawa, Y., Sugino, K., \& Sakurai, H. (2007). Identification of free radical species derived from caffeic acid and related polyphenols. Free Radical Research, 41(1), 110-119. doi:10.1080/10715760600943892

Miura, Y., Inai, M., Honda, S., Masuda, A., \& Masuda, T. (2014). Reducing Effects of Polyphenols on Metmyoglobin and the in Vitro Regeneration of Bright Meat Color by Polyphenols in the Presence of Cysteine. Journal of agricultural and food chemistry(39), 9472-9478.

Murata, M., Sugiura, M., Sonokawa, Y., Shimamura, T., \& Homma, S. (2002). Properties of chlorogenic acid quinone: Relationship between browning and the formation of hydrogen peroxide from a quinone solution. Bioscience, Biotechnology, and Biochemistry (Japan)(12), 2525-2530.

Namiki, M., Yabuta, G., Koizumi, Y., \& Yano, M. (2001). Development of free radical products during the greening reaction of caffeic acid esters (or chlorogenic acid) and a primary amino compound. Bioscience, Biotechnology, and Biochemistry (Japan)(10), 2131-2136.

Ningjian, L., \& David, D. K. (2015). Role of Chlorogenic Acids in Controlling Oxidative and Inflammatory Stress Conditions. Nutrients, 8(1), 16-35. doi:10.3390/nu8010016

Ooms, N., Jansens, K. J. A., Pareyt, B., Reyniers, S., Brijs, K., \& Delcour, J. A. (2018). The impact of disulfide bond dynamics in wheat gluten protein on the development of fermented pastry crumb. Food Chemistry, 242, 68-74. doi:10.1016/j.foodchem.2017.09.007

Panera, B. (2018). The No No List, Panera bread. Retrieved from https://www.panerabread.com/panerabread/documents/panera-no-no-list-05-2015.pdf

Penalver, M. J., Rodriguez-Lopez, J. N., Garcia-Molina, F., Garcia-Canovas, F., \& Tudela, J. (2002). Method for the determination of molar absorptivities of thiol adducts formed from diphenolic substrates of polyphenol oxidase. Analytical Biochemistry(2), 180-185.

Pierpoint, W. S. (1966). The enzymic oxidation of chlorogenic acid and some reactions of the quinone produced. Biochem J, 2(98), 567-580.

Pierpoint, W. S. (1969). O-Quinones formed in plant extracts; their reactions with amino acids and peptides. Bio-chemical journal(112), 609-616.

Prigent, S. V. E., Voragen, A. G. J., Li, F., Visser, A. J. W. G., Koningsveld, v., G.A., \& Gruppen, H. (2008). Covalent interactions between amino acid side chains and oxidation products of caffeoylquinic acid (chlorogenic acid). Journal of the Science of Food and Agriculture, 88, 1748-1754.

Radiana Maria, T. B., Ciprian Nicolae, P., \& Stela, P. (2013). Assessment of the E920 additive (L-cysteine) in relation to some problems of modern food industry. Scientific Papers Series : Management, Economic Engineering in Agriculture and Rural Development, 13(1), 413-418.

Rawel, H. M., \& Rohn, S. (2010). Nature of hydroxycinnamate-protein interactions. Phytochemistry Reviews(1), 93-109.

Richard, F. C., Goupy, P. M., Nicolas, J. J., Lacombe, J. M., \& Pavia, A. A. (1991). Cysteine as an inhibitor of enzymatic browning. 1. Isolation and characterization of addition compounds formed during oxidation of phenolics by apple polyphenol oxidase. Journal of agricultural and food chemistry (USA)(5), 841-849.

Richard-Forget, F. C., Goupy, P. M., \& Nicolas, J. J. (1992). Cysteine as an inhibitor of enzymatic browning. 2. Kinetic studies. Journal of agricultural and food chemistry (USA), 40(11), 2108-2113.

Rogers, A., Lan, H., Vu, P., \& Were, L. (2018a). Greening in sunflower butter cookies as a function of egg replacers and baking temperature. In (Vol. 55, pp. 1478-1488).

Rogers, A., Lan, H., Vu, P., \& Were, L. (2018b). Greening in sunflower butter cookies as a function of egg replacers and baking temperature. Journal of Food Science and Technology-Mysore, 55, 1478-1488. doi:10.1007/s13197-018-3064-7 
577

578

579

580

581

582

583

584

585

586

587

588

589

590

591

592

593

594

595

596

597

598

599

600

601

602

603

604

605

606

607

608

609

610

611

612

613

614

615

616

617

618

Rong, T. (2010). Chemistry and Biochemistry of Dietary Polyphenols. Nutrients, Vol 2, Iss 12, Pp 1231-1246 (2010)(12), 1231. doi:10.3390/nu2121231

SAS Institute Inc., N. S. U. (2014). Statistical Analysis Software (Version 9.3). Cary, NC, USA: SAS Institute Inc. Retrieved from https://www.stat.ncsu.edu/research/sas/citationRetrieved from https://www.sas.com/en us/home.html

SAS, I. I. (2014). Statistical Analysis Software (Version SAS/ACCESS ${ }^{\circledR}$ 9.3). Cary, NC, USA: SAS Institute Inc. Retrieved from https://www.sas.com/en us/home.html

Schilling, S., Sigolotto, C. I., Carle, R., \& Schieber, A. (2008). Characterization of covalent addition products of chlorogenic acid quinone with amino acid derivatives in model systems and apple juice by high-performance liquid chromatography/electrospray ionization tandem mass spectrometry. Rapid Communications In Mass Spectrometry(4), 441-448.

Schlichtherle-Cerny, H., \& Amado, R. (2002). Analysis of Taste-Active Compounds in an Enzymatic Hydrolysate of Deamidated Wheat Gluten. Journal of Agricultural and Food Chemistry(6), 1515-1528.

Snell, J. M., \& Weissberger, A. (1939). Reaction of thiol compounds with quinones. Journal of the American Chemical Society, 61, 450-453.

Timbo, B., Koehler, K. M., Wolyniak, C., \& Klontz, K. C. (2004). Sulfites--a Food and Drug Administration review of recalls and reported adverse events. Journal of food protection(8).

Tosovic, J., Markovic, S., Markovic, J. M. D., Mojovic, M., \& Milenkovic, D. (2017). Antioxidative mechanisms in chlorogenic acid. Food Chemistry, 237, 390-398. doi:10.1016/j.foodchem.2017.05.080

Whole Foods Market, I. L. P. (2018). Whole Foods Market's Unacceptable Ingredients \& Food. Retrieved from https://www.wholefoodsmarket.com/values-matter/unacceptableingredients-food

Yabuta, G., Koizumi, Y., Namiki, K., Hida, M., \& Namiki, M. (2001). Structure of green pigment formed by the reaction of caffeic acid esters (or chlorogenic acid) with a primary amino compound. Bioscience, Biotechnology, and Biochemistry (Japan), 65(10), 2121-2130.

Yu, L., Nanguet, A.-L., \& Beta, T. (2013). Comparison of Antioxidant Properties of Refined and Whole Wheat Flour and Bread. Antioxidants(4), 370-383. doi:10.3390/antiox2040370

Zeng, X., Bai, W., Zhu, X., \& Dong, H. (2017). Browning Intensity and Taste Change Analysis of Chicken Protein-Sugar Maillard Reaction System with Antioxidants and Different Drying Processes. Journal of Food Processing \& Preservation, 41(2), 1-7. doi:10.1111/jfpp.13117

Zhang, Z., Elfalleh, W., He, S., Tang, M., Zhao, J., Wu, Z., . . . Sun, H. (2018). Heating and cysteine effect on physicochemical and flavor properties of soybean peptide Maillard reaction products. International Journal of Biological Macromolecules, 120(Part B), 2137-2146. doi:10.1016/j.ijbiomac.2018.09.082 


\section{6. Figure Captions}

620 Fig 1 Effect of cysteine on browning measured by absorbance at $410 \mathrm{~nm}$ (A, B,

$621 \longrightarrow$ Control, $--1.02 \mathrm{mM}$ Cysteine,$--\nabla-+1.27 \mathrm{mM}$ Cysteine,

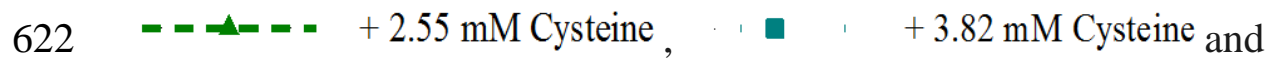

623 -

$624 \longrightarrow$ Control,$-\rightarrow-+1.02 \mathrm{mM}$ Cysteine,,$-\boldsymbol{-}-+1.27 \mathrm{mM}$ Cysteine,

$625-=- \pm=-+2.54 \mathrm{mM}$ Cysteine, , - i $\quad+3.82 \mathrm{mM}$ Cysteine and

$626 \quad-\square-\quad+5.09 \mathrm{mM}$ Cysteine $)$ and photos representing visual appearance with

627 corresponding correlation coefficients of $\mathrm{pH} 8$ and 9 buffered chlorogenic acid (CGA):

628 lysine: cysteine solutions incubated at ambient temperature. Data presented were means \pm

629 standard deviations of 3 replicates. Means with the same letter after $48 \mathrm{~h}$ were not

630 significantly different.

631 Fig 2 Postulated cysteine inhibition mechanism of chlorogenic acid (CGA) quinone-

632 lysine greening at alkaline $\mathrm{pH} . \mathrm{R}_{1}, \mathrm{R}_{2}$, and $\mathrm{R}_{3}$ refers to a quinic acid, lysine and cysteine

633 moiety, respectively. The CGA quinone derivatives may be monomer, dimer or

634 polymers. Dash-line framed route was adapted from Yabuta et al (2001), which was a

635 potential but less predominant route.

636 Fig 3 Postulated mechanism of cysteine regenerating chlorogenic acid hydroquinone

637 dimer by redox reaction.

638 Fig 4 Mass spectrometric chromatograms and chemical structures of identified

639 compounds in pH 8 and 9 chlorogenic acid: Lys: Cys solutions. A to G corresponding to 640 compound 1 to 7 in Table 1. 
641 Fig 5 Trolox equivalents antioxidant capacity (A) and gallic acid equivalent reducing

642 capacity (B) as a function of cysteine concentration (0 - $5.091 \mathrm{mM})$ in buffered

$643 \multimap$ pH 8 and $\multimap-0$ pH 9 CGA: Lys: Cys solutions. 
A. Browning in pH 8 buffered CGA: lysine: cysteine solutions incubated at ambient temperature for $48 \mathrm{~h}$

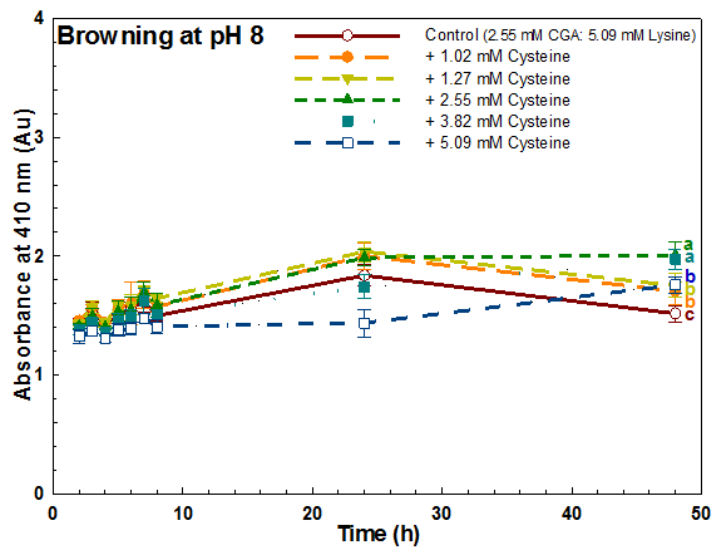

C. Greening in pH 8 buffered CGA: lysine: cysteine solutions incubated at ambient temperature for $48 \mathrm{~h}$
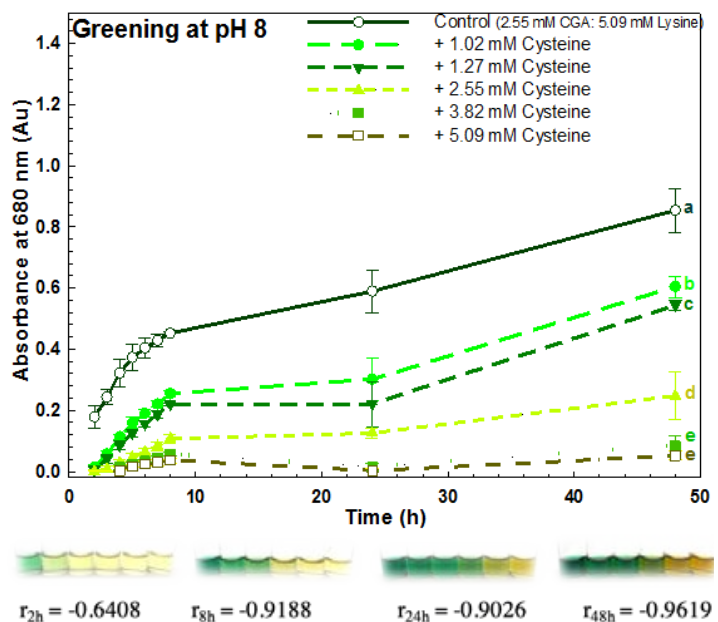

B. Browning in $\mathrm{pH} 9$ buffered CGA: lysine: cysteine solutions incubated at ambient temperature for $48 \mathrm{~h}$

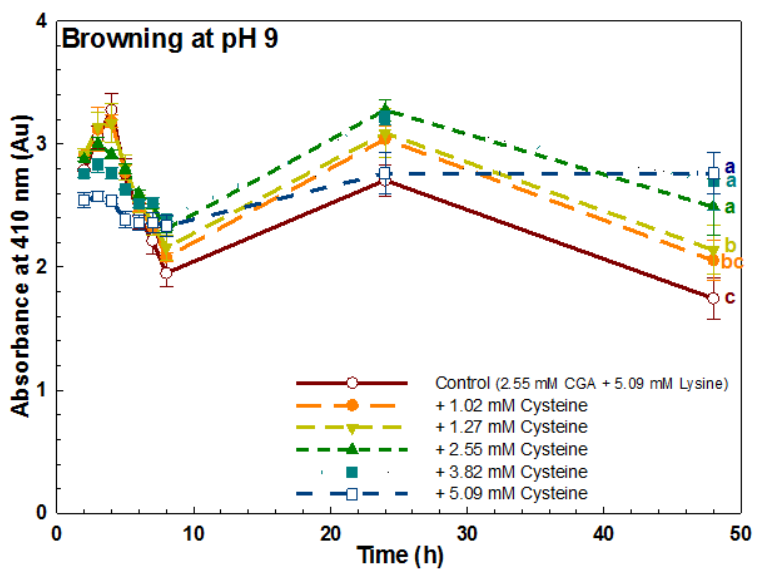

D. Greening in pH 9 buffered CGA: lysine: cysteine solutions incubated at ambient temperature for $48 \mathrm{~h}$

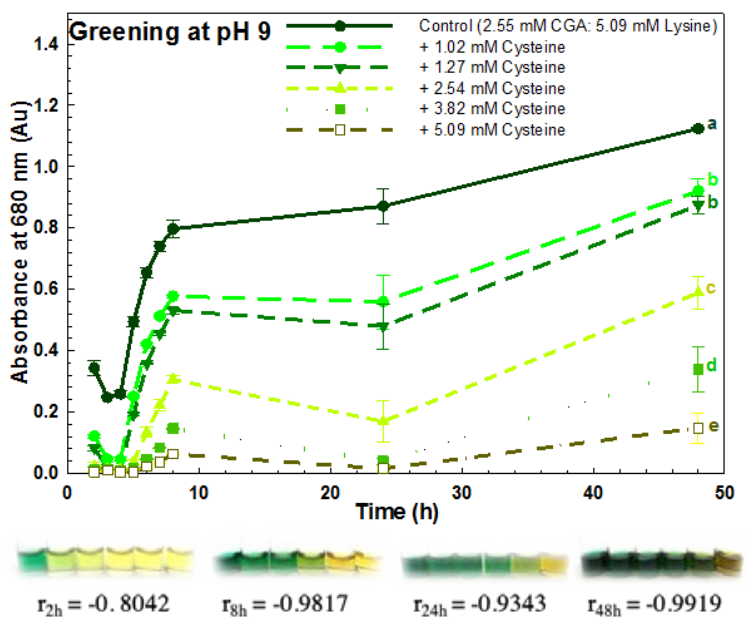

$644 \quad$ Fig 1 


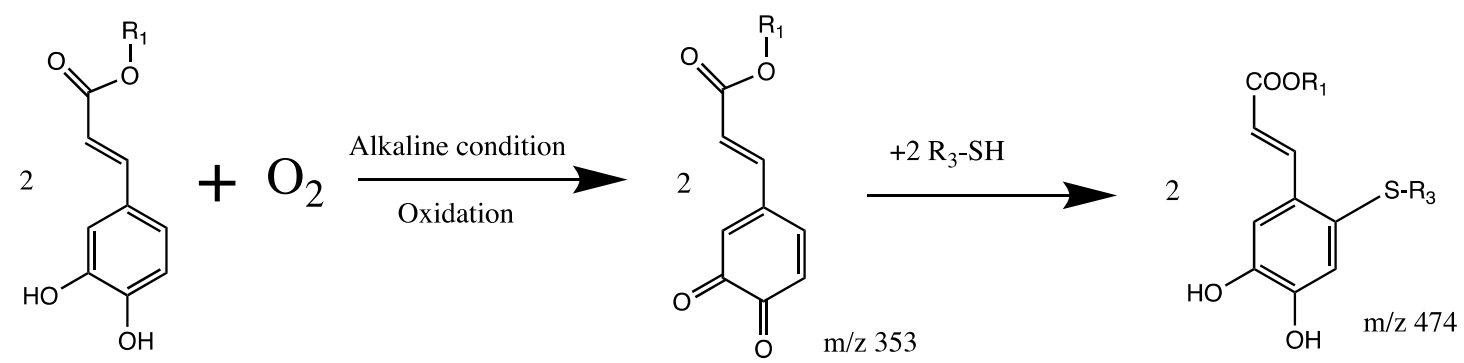

$\mathrm{m} / \mathrm{z} 354$

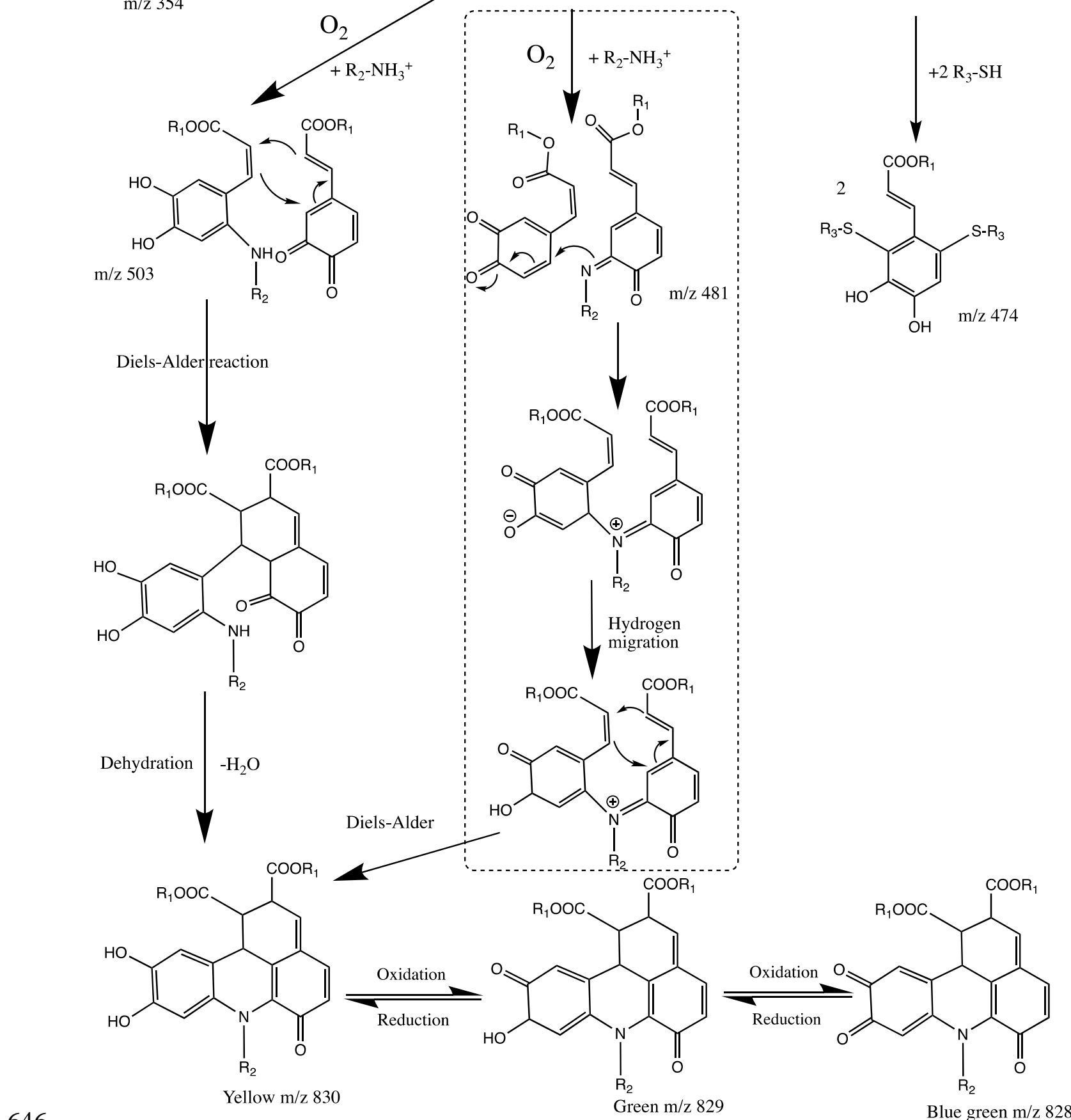

Fig 2 


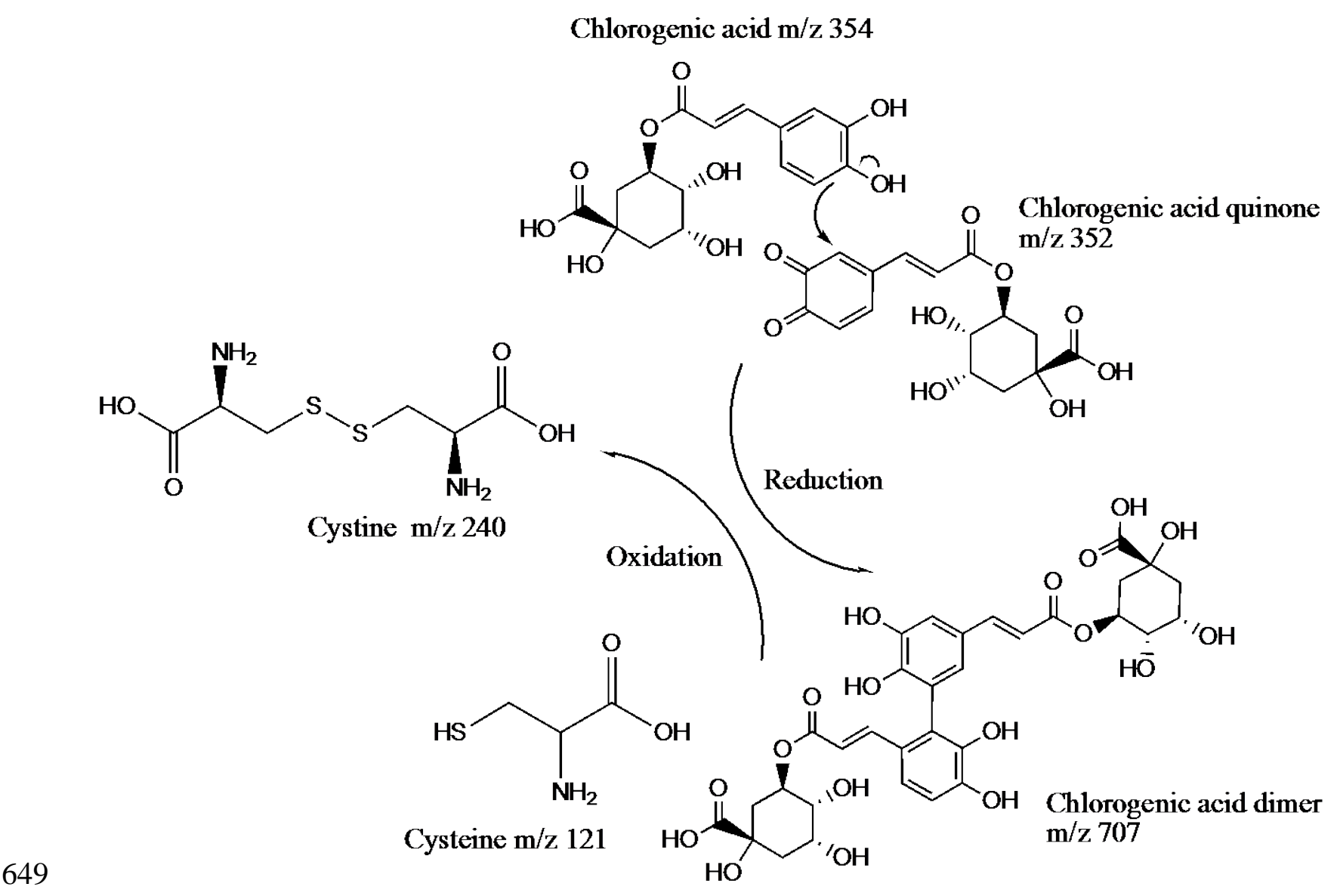

$650 \quad$ Fig 3

651 
A. Compound 1: Cysteinyl-CGA conjugate $(\mathrm{m} / \mathrm{z} 475)$ minus a carboxyl group (44 Da)

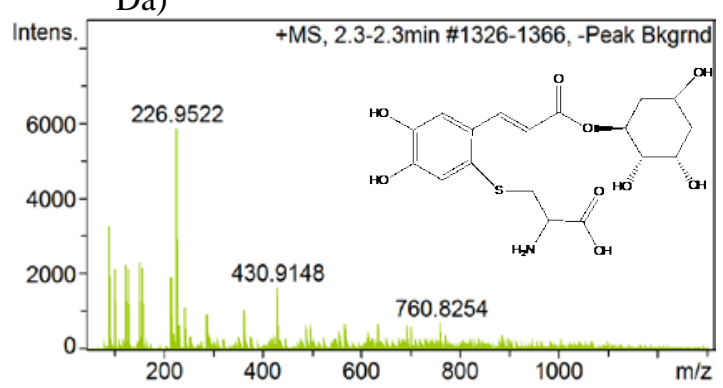

C. Compound 3: Mono-cysteinyl CGA crosslink (m/z 947)

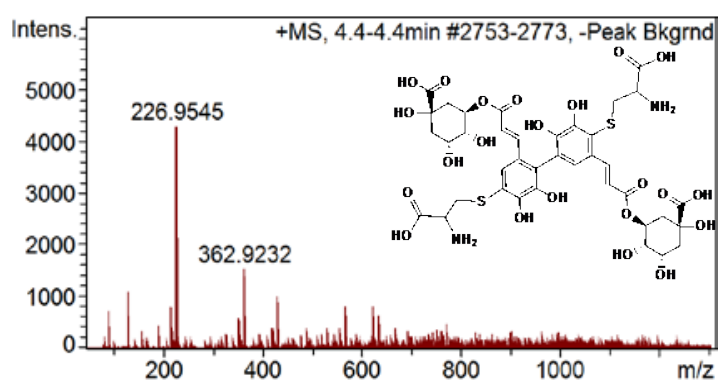

E. Trihydroxy benzacridine $(\mathrm{m} / \mathrm{z} 830)$ minus

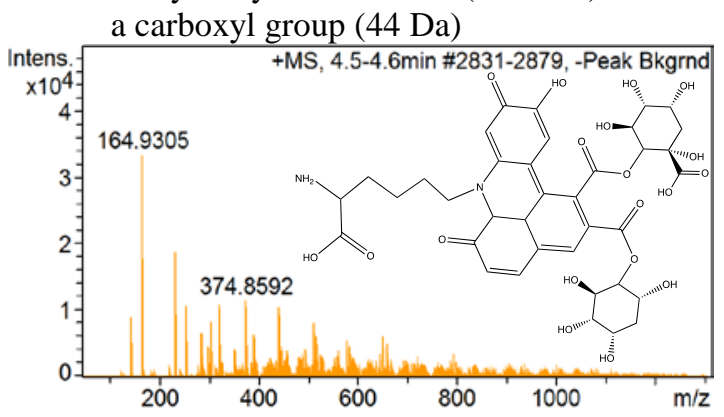

B. Compound 2: Lysyl amine-CGA conjugate $(\mathrm{m} / \mathrm{z} 504)+\left[\mathrm{Na}^{+}\right]$

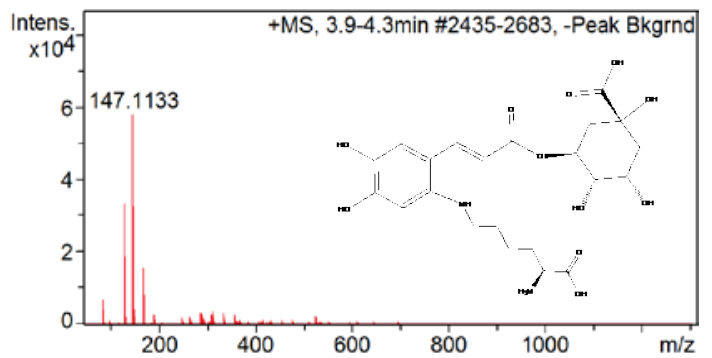

D. Compound 4: Di-cysteinyl CGA conjugate (m/z 595) + carboxyl group (44 $\mathrm{Da})+\left[\mathrm{Na}^{+}\right]$

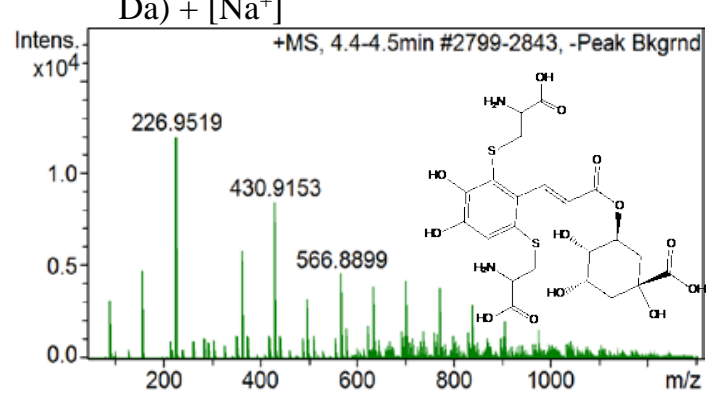

F. Cystine-cystine crosslink (m/z 481)

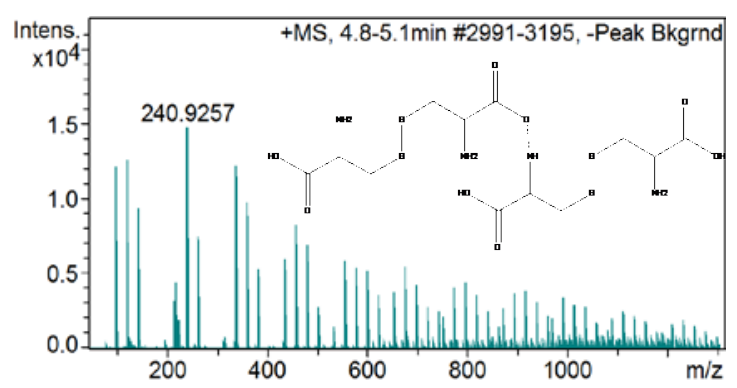

\section{$652 \quad$ Fig 4}

G. Chlorogenic acid dimer $(\mathrm{m} / \mathrm{z} 708)+\left[\mathrm{Na}^{+}\right]$

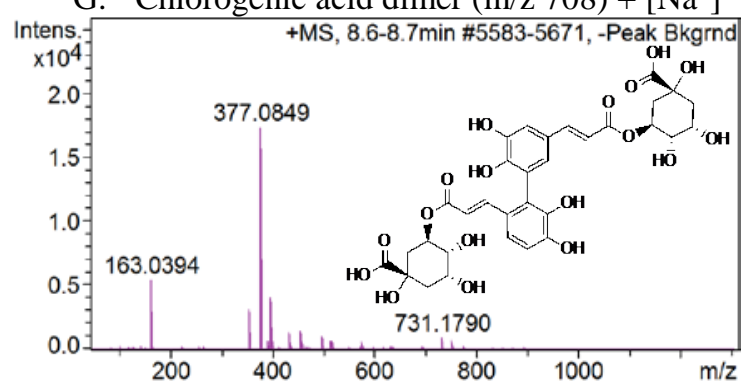

653 
D. Trolox equivalent antioxidant capacity (mM) as a function of cysteine concentration

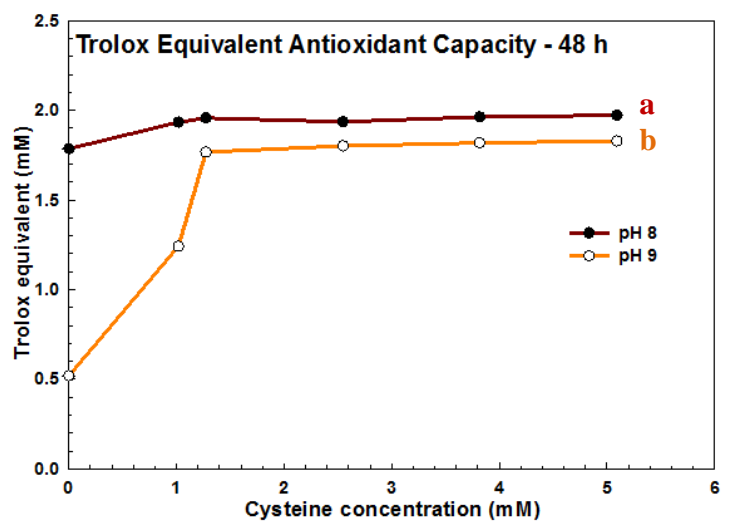

E. Gallic acid equivalent reducing capacity $(\mathrm{mg} / \mathrm{mL})$ as a function of cysteine concentration

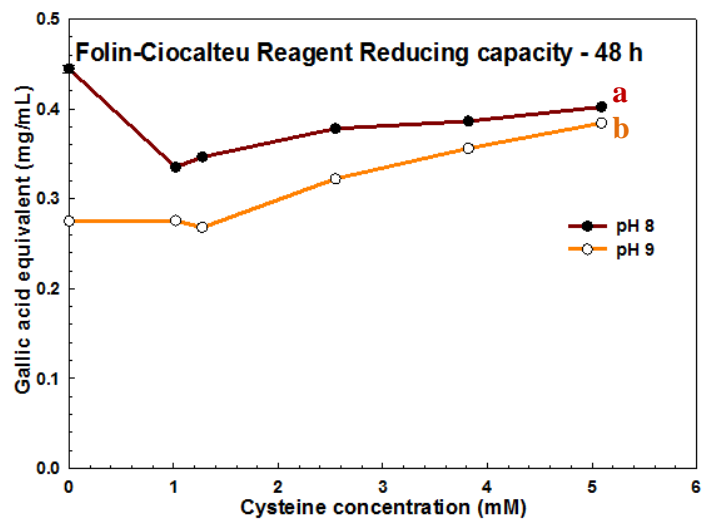

F. Free CGA (mM) as a function of cysteine concentration

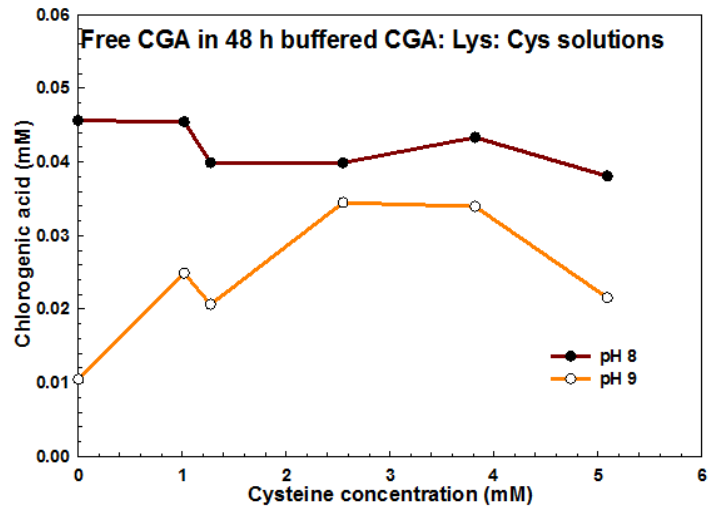

$654 \quad$ Fig 5

655 
656 Table 1 Identification of predominant reaction products (signal intensity $>4000$,

657 signal/noise ratio>10) in $\mathrm{pH} 8$ and 9 buffered chlorogenic acid /CGA: Lys: Cys solutions

658 after $48 \mathrm{~h}$ incubation.

\begin{tabular}{|c|c|c|c|c|}
\hline Compound & $\begin{array}{l}\text { Retention } \\
\text { time (min) }\end{array}$ & {$[\mathbf{M}+\mathbf{H}]^{+}$} & $\begin{array}{l}\text { MS fragments } \\
\text { (\% relative abundance) }\end{array}$ & $\begin{array}{l}\text { Tentative } \\
\text { identification }\end{array}$ \\
\hline 1 & 2.3 & 430.91 & $\begin{array}{l}81(18.1), 91(56.6), 159 \\
(29.0), 217(55.4), 219 \\
(17.1), 227(100), 243 \\
(14.2), 363(13.8), 421 \\
(13.2), 431(12.6)\end{array}$ & $\begin{array}{l}\text { Cysteinyl-CGA } \\
\text { conjugate (475) - } \\
\text { Carboxyl group (44) }\end{array}$ \\
\hline 2 & 4.1 & 527.25 & $\begin{array}{l}84(12.1), 130(55.6), \\
147(100), 148(7.1), \\
169(26.6), 191(4.5), \\
289(5.3), 311(5.2), 359 \\
(4.1), 527(4.0)\end{array}$ & $\begin{array}{l}\text { Lysine-CGA } \\
\text { conjugates }(504)+ \\
{\left[\mathrm{Na}^{+}\right]}\end{array}$ \\
\hline 3 & 4.4 & 946.84 & $\begin{array}{l}91(33.2), 159(16), 227 \\
(100), 363(19.9), 431 \\
(28.2), 499(8.9), 567 \\
(10.6), 635(12.7), 703 \\
(12.3), 947(6.9)\end{array}$ & $\begin{array}{l}\text { Cysteinyl-CGA- } \\
\text { Cysteinyl-CGA } \\
\text { crosslink (947) }\end{array}$ \\
\hline 4 & 4.5 & 662.82 & $\begin{array}{l}91(40.8), 159(33), 227 \\
(100), 255(14.8), 323 \\
(26.3), 391(18.9), 459 \\
(23.2), 527(24.4), 595 \\
(24.4), 663(18.6)\end{array}$ & $\begin{array}{l}\text { Di-cysteinyl-CGA } \\
\text { conjugate (595) }+ \\
\text { Carboxyl group (44) + } \\
{\left[\mathrm{Na}^{+}\right]}\end{array}$ \\
\hline 5 & 4.6 & 786.71 & $\begin{array}{l}143 \text { (100), } 165 \text { (86.1), } \\
263 \text { (70.5), } 285 \text { (56.0), } \\
383(44.5), 405(65.8), \\
525 \text { (49.0), } 645(48.7), \\
667 \text { (40.2), } 787(40.5)\end{array}$ & $\begin{array}{l}\text { Trihydroxy } \\
\text { benzacridine (830) - } \\
\text { Carboxyl group (44) }\end{array}$ \\
\hline 6 & 5 & 480.84 & $\begin{array}{l}86 \text { (72.5), } 99(100), 100 \\
(44.1), 125 \text { (58.6), } 146 \\
\text { (42.9), } 241(58.0), 263 \\
\text { (56.6), } 361(45.3), 459 \\
(42.7), 481 \text { (51.6) }\end{array}$ & $\begin{array}{l}\text { Cystine-cystine } \\
\text { crosslink (480) }\end{array}$ \\
\hline 7 & 8.7 & 731.18 & $\begin{array}{l}163 \text { (31.4), } 164 \text { (4.5), } \\
225(6.6), 355 \text { (24.9), } \\
377 \text { (100), } 378(13.0), \\
399 \text { (27.5), } 435(5.7), \\
457 \text { (7.0), } 731(9.6)\end{array}$ & $\begin{array}{l}\text { Chlorogenic acid } \\
\text { dimer }(708)+\left[\mathrm{Na}^{+}\right]\end{array}$ \\
\hline
\end{tabular}

659

660 
G. pH 8 buffered CGA: Lys: Cys solutions absorbance at $320 \mathrm{~nm}$

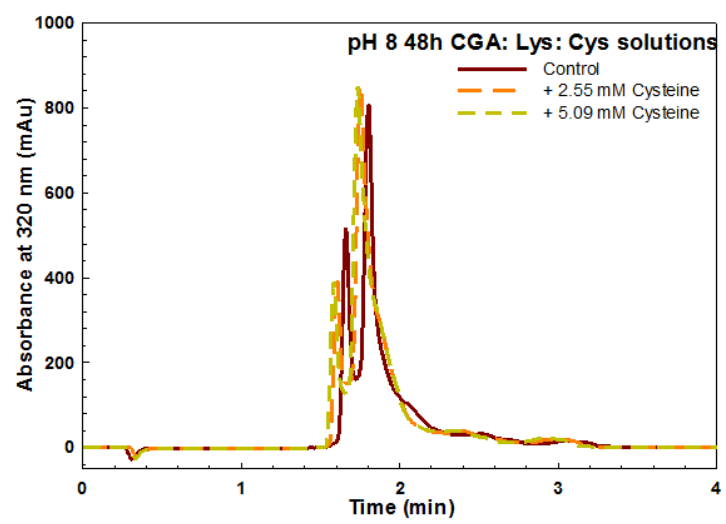

I. pH 8 buffered CGA: Lys: Cys solutions absorbance at $450 \mathrm{~nm}$

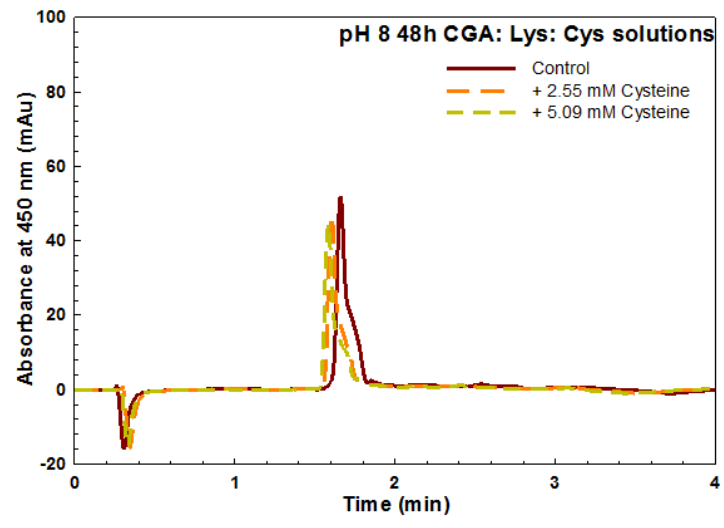

K. pH 8 buffered CGA: Lys: Cys solutions absorbance at $680 \mathrm{~nm}$

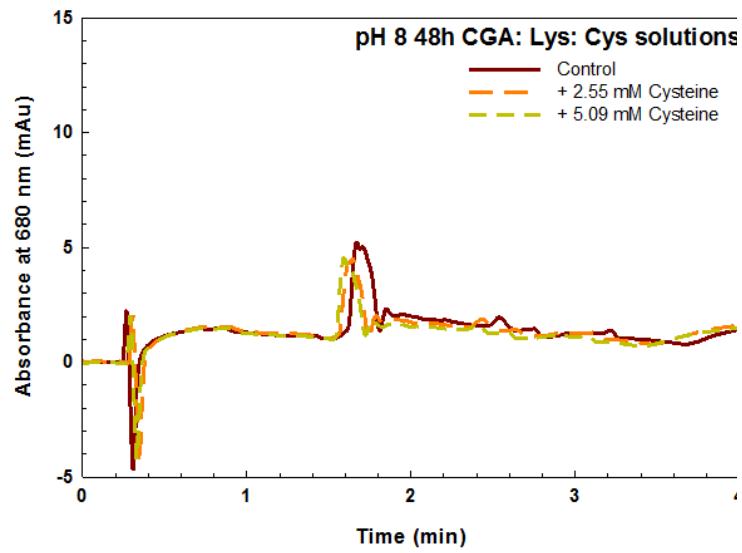

H. pH 9 buffered CGA: Lys: Cys solutions absorbance at $320 \mathrm{~nm}$

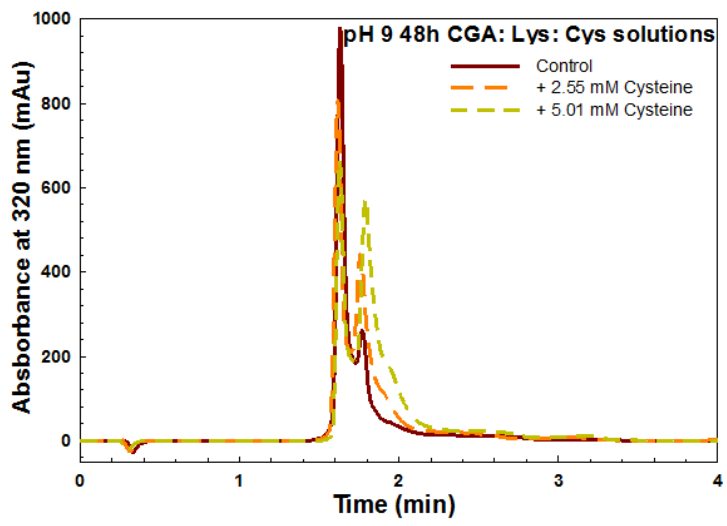

J. $\quad$ pH 9 buffered CGA: Lys: Cys solutions absorbance at $450 \mathrm{~nm}$

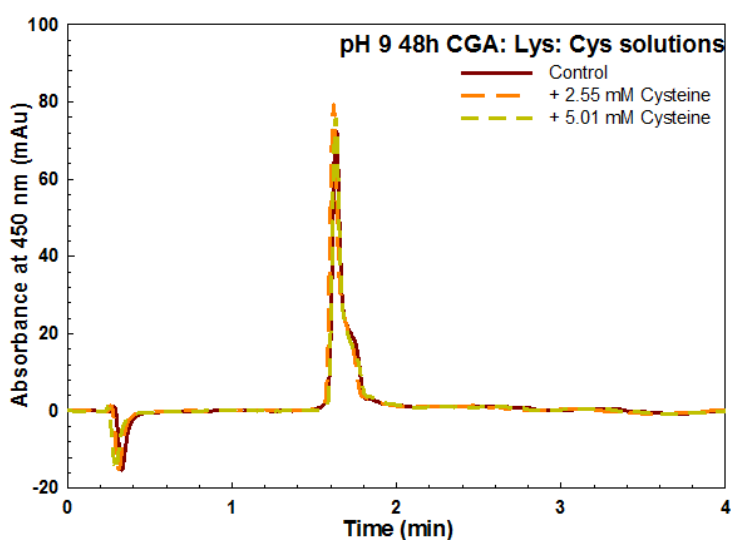

L. pH 9 buffered CGA: Lys: Cys solutions absorbance at $680 \mathrm{~nm}$

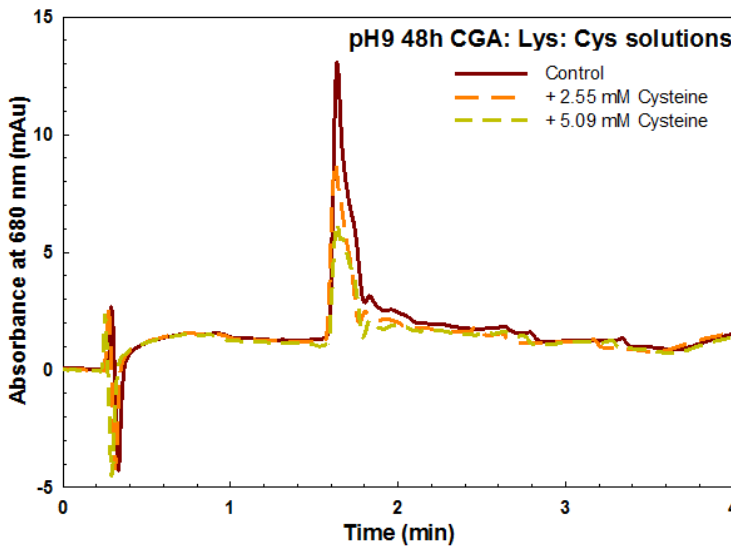

662 Fig S1 HPLC chromatograms at $320 \mathrm{~nm}$ (A, B), $450 \mathrm{~nm}$ (C, D), and $680 \mathrm{~nm}$ (E, F) of pH

6638 and 9 buffered CGA: Lys: Cys solutions ( Control,

$664-+2.55 \mathrm{mM}$ Cysteine $---+5.01 \mathrm{mM}$ Cysteine ) after 48 hours

665 incubation at ambient temperature. 
C. Browning intensity in $\mathrm{pH} 7.75,8$ and 9 buffered CGA: lysine: cysteine solutions

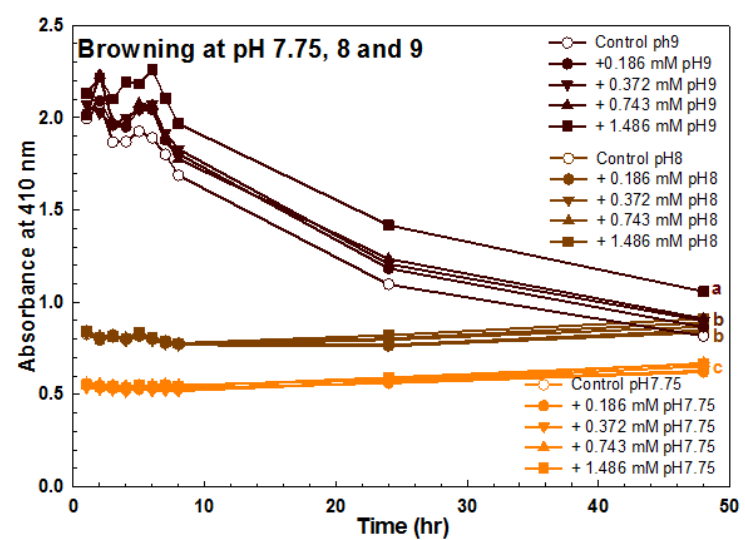

D. Greening intensity in $\mathrm{pH} 7.75,8$ and 9 buffered CGA: lysine: cysteine solutions

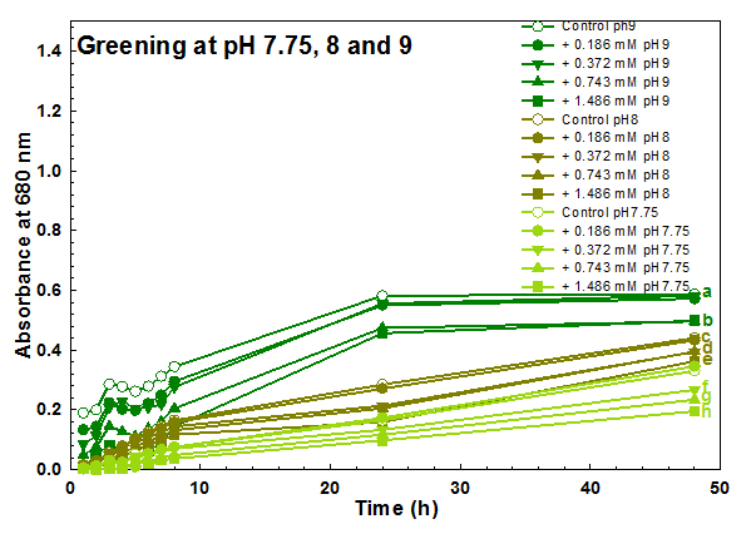

666 Fig S2 Browning measured by absorbance at $410 \mathrm{~nm}$ (A) and greening measured by absorbance at $680 \mathrm{~nm}$ (B) in $\mathrm{pH}$ 7.75, 8 and 9 buffered CGA: lysine: cysteine solutions as

668 a function of reaction time when cysteine increased from 0 to $1.486 \mathrm{mM}$. The data 669 presented were means of 3 replicates. Means with the same letter were not significantly 670 different after $48 \mathrm{~h}$.

671 
672 Table S1 Relative abundance of eluted compounds at 1.60 and 1.75 minutes by HPLC 673 peak area at $320 \mathrm{~nm}$.

\begin{tabular}{|c|c|c|c|c|c|c|}
\hline \multirow[b]{2}{*}{$\begin{array}{l}\text { Cysteine } \\
(\mathrm{mM})\end{array}$} & \multicolumn{2}{|l|}{ pH 8} & \multicolumn{3}{|c|}{ pH 9} & \multirow[b]{2}{*}{$48 \mathrm{~h}$} \\
\hline & $8 \mathrm{~h}$ & $24 \mathrm{~h}$ & $48 \mathrm{~h}$ & $8 \mathrm{~h}$ & $24 \mathrm{~h}$ & \\
\hline \multicolumn{7}{|c|}{$\begin{array}{l}\text { Compound eluted at } 1.60 \text { minutes which was determined to be green trihydroxy } \\
\text { benzacridine derivatives }\end{array}$} \\
\hline 0 & 740.57 & 1457.23 & 1870.00 & 3268.67 & 3457.27 & 3891.87 \\
\hline 1.02 & 710.26 & 1345.35 & 1736.49 & 2979.39 & 3414.17 & 3385.75 \\
\hline 1.27 & 683.81 & 1405.27 & 1701.61 & 2834.30 & 3281.49 & 3371.49 \\
\hline 2.55 & 832.99 & 1457.65 & 1411.35 & 2287.25 & 3049.73 & 3123.02 \\
\hline 3.82 & 953.79 & 1414.71 & 1434.47 & 2036.34 & 2682.04 & 2785.24 \\
\hline 5.09 & 896.76 & 1214.52 & 1539.28 & 1885.01 & 2519.79 & 2657.22 \\
\hline \multicolumn{7}{|c|}{$\begin{array}{l}\text { Compound eluted at } 1.75 \text { minutes which was suspected to be amine/thiol-CGA } \\
\text { conjugates }\end{array}$} \\
\hline 0 & $1.03 \mathrm{E}+04$ & 7382.13 & 6300.74 & 5348.54 & 2365.87 & 2284.20 \\
\hline 1.02 & $1.14 \mathrm{E}+04$ & 7450.91 & 6426.08 & 5552.52 & 3601.03 & 2574.61 \\
\hline 1.27 & $1.05 E+04$ & 8244.17 & 7048.81 & 4135.43 & 2738.76 & 3101.64 \\
\hline 2.55 & $1.09 \mathrm{E}+04$ & 8299.54 & 7821.39 & 6666.02 & 4958.46 & 3996.77 \\
\hline 3.82 & $1.10 \mathrm{E}+04$ & $1.01 \mathrm{E}+04$ & 8358.80 & 7487.74 & 5222.59 & 4976.34 \\
\hline 5.09 & $1.13 E+04$ & 8313.35 & 7971.60 & 8352.71 & 6646.72 & 5647.92 \\
\hline
\end{tabular}

674

675 
676 Table S2 General linear model effect and interaction of $\mathrm{pH}$, cysteine concentration and 677 incubation time on color intensities and antioxidant capacity.

\begin{tabular}{|c|c|c|c|c|c|}
\hline $\begin{array}{l}\text { Independent } \\
\text { variables }\end{array}$ & & $\begin{array}{l}\text { Greening } \\
\left(\text { A680 }_{\mathrm{nm}}\right)\end{array}$ & $\begin{array}{l}\text { Browning } \\
(\text { (A410 nm) }\end{array}$ & $\begin{array}{l}\text { Trolox } \\
\text { equivalent } \\
\text { antioxidant } \\
\text { capacity }\end{array}$ & $\begin{array}{l}\text { Folin-Ciocalteu } \\
\text { reagent reducing } \\
\text { capacity }\end{array}$ \\
\hline \multirow[t]{4}{*}{ Cysteine } & $\mathrm{df}$ & 5 & 5 & 5 & 5 \\
\hline & $\mathrm{ms}$ & 1.780 & 0.361 & 0.858 & 0.015 \\
\hline & $\mathrm{P}$ & $<0.0001$ & $<0.0001$ & $<0.0001$ & $<0.0001$ \\
\hline & $\mathrm{S}$ & $* * * *$ & $* * * *$ & $* * * *$ & $* * * *$ \\
\hline \multirow[t]{4}{*}{$\mathrm{pH}$} & $\mathrm{df}$ & 1 & 1 & 1 & 1 \\
\hline & $\mathrm{ms}$ & 1.027 & 87.617 & 2.244 & 0.063 \\
\hline & $\mathrm{P}$ & $<0.0001$ & $<0.0001$ & $<0.0001$ & $<0.0001$ \\
\hline & $\mathrm{S}$ & $* * * *$ & $* * * *$ & $* * * *$ & $* * * *$ \\
\hline \multirow[t]{4}{*}{ Time } & $\mathrm{df}$ & 9 & 9 & 2 & 2 \\
\hline & $\mathrm{ms}$ & 0.903 & 1.915 & 0.139 & 0.028 \\
\hline & $\mathrm{P}$ & $<0.0001$ & $<0.0001$ & $<0.0001$ & $<0.0001$ \\
\hline & $\mathrm{S}$ & $* * * *$ & $* * * *$ & $* * * *$ & $* * * *$ \\
\hline \multirow{4}{*}{$\begin{array}{l}\text { Interaction } \\
\text { (pH*cysteine) }\end{array}$} & $\mathrm{df}$ & 5 & 5 & 5 & 5 \\
\hline & $\mathrm{ms}$ & 0.062 & 0.041 & 1.536 & 0.035 \\
\hline & $\mathrm{P}$ & $<0.0001$ & $<0.0001$ & $<0.0001$ & $<0.0001$ \\
\hline & $\mathrm{S}$ & $* * * *$ & $* * * *$ & $* * * *$ & $* * * *$ \\
\hline \multirow{4}{*}{$\begin{array}{l}\text { Interaction } \\
\text { (cysteine*time) }\end{array}$} & $\mathrm{df}$ & 45 & 45 & 10 & 10 \\
\hline & $\mathrm{ms}$ & 0.060 & 0.099 & 0.295 & 0.001 \\
\hline & $\mathrm{P}$ & $<0.0001$ & $<0.0001$ & $<0.0001$ & $<0.0001$ \\
\hline & $\mathrm{S}$ & $* * * *$ & $* * * *$ & $* * * *$ & $* * * *$ \\
\hline \multirow{4}{*}{$\begin{array}{l}\text { Interaction } \\
\text { (pH*time) }\end{array}$} & $\mathrm{df}$ & 9 & 9 & 2 & 2 \\
\hline & $\mathrm{ms}$ & 0.084 & 1.861 & 0.331 & 0.003 \\
\hline & $\mathrm{P}$ & $<0.0001$ & $<0.0001$ & $<0.0001$ & $<0.0001$ \\
\hline & $\mathrm{S}$ & $* * * *$ & $* * * *$ & $* * * *$ & $* * * *$ \\
\hline \multirow{4}{*}{$\begin{array}{l}\text { Interaction } \\
\text { (pH*trt*time) }\end{array}$} & $\mathrm{df}$ & 45 & 45 & 10 & 10 \\
\hline & $\mathrm{ms}$ & 0.007 & 0.044 & 0.664 & 0.002 \\
\hline & $\mathrm{P}$ & $<0.0001$ & $<0.0001$ & $<0.0001$ & $<0.0001$ \\
\hline & $\mathrm{S}$ & $* * * *$ & $* * * *$ & $* * * *$ & $* * * *$ \\
\hline
\end{tabular}

678 df refers to degrees of freedom; ms refers to mean square; P refers to p value; $\mathrm{S}$ refers to 679 the significant level: **** refers to high significance.

680 